\title{
TOPOLOGICAL MODULES: BANACH ALGEBRAS, TENSOR PRODUCTS, ALGEBRAS OF KERNELS $\left({ }^{1}\right)$
}

\author{
BY \\ JESÚS GIL DE LAMADRID( $\left.{ }^{2}\right)$
}

1. Introduction. The unifying idea of the present work is a complex normed (not necessarily complete) vector space $M$ which is a left module over a Banach algebra $A$ and a right module over a Banach algebra $B$. We assume that the complex scalars associate and commute freely throughout the product $U x V$, $U \in A, x \in M$, and $V \in B$. The product $U x V$ is assumed to be continuous in all three variables with respect to the given norms of the spaces in which the variables range. There are two typical examples that will appear over and over. One is the case of a Banach algebra $A$ and a two-sided, not necessarily closed, ideal $M$ of $A$. In this case $M$ can be considered in a natural way as a left and a right module over $A$. The second example is the space $\mathscr{L}(E, F)$ of all bounded linear transformations $T: E \rightarrow F$. This space is a left module over the algebra $\mathscr{L}(F)$ of all bounded linear operators on $F$ and a right module over the algebra $\mathscr{L}(E)$, where the expression $U T V, U \in \mathscr{L}(F), T \in \mathscr{L}(E, F)$ and $V \in \mathscr{L}(E)$, stands for ordinary composition of transformations. From our point of view the interest in a topological module $M$ derives from the fact (Theorem 2.2) that if the original topology of $M$ is strengthened (made finer) the module operations remain continuous, provided that the resulting new topology turns $M$ into a Banach space.

This fact is systematically applied in $\$ 2$ to our first example of the two-sided ideal $M$ of a Banach algebra $A$ to make a study of topologically simple Banach algebras, culminating in the following structure theorem. It states (roughly; for a precise statement see Theorem 2.5) that if $A^{2}$ is dense in $A$ then the Banach algebra $A$ is topologically simple if and only if it has a continuous faithful representation as a dense two-sided ideal of a topologically simple closed subalgebra of the algebra $\mathscr{L}(E)$, for some Banach space $E$.

The second example of topological modules, the space of bounded linear transformations provides the proper setting for a systematic study of the class of uniform cross norms on the tensor product $E \otimes F$ of two Banach spaces $E$ and $F$. This is the topic which occupies most of our attention in the present work. Uniform cross norms were introduced by Schatten [14], but studied by him to any extent

Presented to the Society, April 22, 1966; received by the editors October 16, 1964.

( ${ }^{1}$ ) Presented in part to the Irvine Conference on Functional Analysis, March 29, 1966. A summary of results in $\$ 88$ and 9 of the present work is appearing in the Proceedings of the Irvine Conference.

(2) Support of this work by National Science Foundation research grants (NSF G-19752 and NSF G-24295) is gratefully acknowledged. 
only for Hilbert spaces. They were exploited by the present author in the study [7], [10] of tensor products of Banach algebras. The concept of uniform cross norm is essentially of a metric character. Since we are interested here primarily in topological properties, we have replaced it by its topological counterpart, which we call a modular norm. Another metric concept of Schatten which has been replaced by a topological notion is that of cross norm. The motivation for these departures from the standard theory is that in the present work we quite often have to replace a given norm on $E \otimes F$ by an equivalent norm with certain desirable properties. The passage from one norm to another may not preserve the metric properties, but will obviously preserve the topological ones.

Consider the space $\mathscr{L}\left(E, F^{\prime}\right)$ of bounded linear transformations of $E$ into the dual (conjugate) space $F^{\prime}$ of $F$, and the algebra $\mathscr{L}^{\#}\left(F^{\prime}\right)$ of all operators on $F^{\prime}$ that are transpose (adjoint) operators of operators on $F$. The space $\mathscr{L}\left(E, F^{\prime}\right)$ is a topological left module over $\mathscr{L}^{\#}\left(F^{\prime}\right)$ and a right module over $\mathscr{L}(E)$. We say that a norm $\alpha$ on $E \otimes F$ is modular if the dual space $\left(E \otimes_{\alpha} F\right)^{\prime}$ can be imbedded, in a natural way, in $\mathscr{L}\left(E, F^{\prime}\right)$ in such a way that it becomes a submodule of $\mathscr{L}\left(E, F^{\prime}\right)$ under the indicated structure. This definition was essentially obtained by adopting the algebraic part of Schatten's characterization [14, Theorem 4.1, p. 62], of uniform cross norms and leaving out the metric part. It will turn out, and will be a major theme in the present work, that, although the notion of modular norm is purely algebraic, it contains all the essential topological ingredients associated with uniform cross norms. The principal manifestation of this phenomenon occurs in Theorem 4.2. Most norms on tensor products, which occur here, are modular.

In $\$ 3$ we collect the principal known notations, definition, conventions, and results for topological tensor products that will be used in the rest of this work. The exposition is based on the work of Schatten [14] and of Grothendieck [11].

In $\$ 4$ we begin the systematic study of the tensor product $E \otimes_{\alpha} F$ for a modular norm $\alpha$. The concept of admissible norm appears. A norm $\alpha$ is admissible if it majorizes $\lambda$ and is majorized by $\gamma$. This is the topological counterpart of norms which Schatten used, satisfying the metric condition $\lambda \leqq \alpha \leqq \gamma$, and which we have called [10] ordinary norms. A modular norm turns out to be admissible, (Theorem 4.5) a result which seems to be new even for uniform norms. The result actually boils down to the fact that, for modular norms, the dual space $\left(E \otimes_{\alpha} F\right)^{\prime}$ is contained in $\mathscr{L}\left(E, F^{\prime}\right)$ and contains the space of all bounded linear transformations of $E$ into $F^{\prime}$ of finite rank. Since this last space is isomorphic to $E^{\prime} \otimes F^{\prime}$, the dual norm $\alpha^{\prime}$ on $\left(E \otimes_{\alpha} F\right)^{\prime}$ induces a norm on $E^{\prime} \otimes F^{\prime}$, which we call again $\alpha^{\prime}$. The norm $\alpha^{\prime}$ turns out to be also admissible (Theorem 4.4), but may not be modular, unless (Theorem 4.8) $E$ is reflexive and $\alpha$ is strongly modular. This last concept means that $\alpha$ is not only modular, but that $\left(E \otimes_{\alpha} F\right)^{\prime}$ remains invariant under left multiplication by elements of the entire algebra $\mathscr{L}\left(F^{\prime}\right)$, not merely those of $\mathscr{L}^{\#}\left(F^{\prime}\right)$.

Next we consider the Kronecker product $V \otimes W$ of two operators $V \in \mathscr{L}(E)$ 
and $W \in \mathscr{L}(F)$. This is defined as a linear operator on $E \otimes F$ in such a way that it generalizes the classical Kronecker product of matrices. The term is our own, but the resulting operator was used by Schatten in connection with uniform cross norms and by Grothendieck in connection with the locally-convex-space equivalent of the $\gamma$ norm. The Kronecker product is the basic tool in the work of the present author in [7] and [10] on topological tensor products of Banach algebras. In the present work it is established (Theorems 4.6 and 4.7) that modular norms provide the proper setting for extending the algebraic operator $V \otimes W$ on the algebraic tensor product $E \otimes F$ to an element of $\mathscr{L}\left(E \otimes_{\alpha} F\right)$.

We postpone for the moment the discussion of the content of $\S 5$ below, and pass on to the discussion of $\S 6$, which perhaps contains the basic results of this work. We shall assume for the purpose of this summary that $\alpha$ is a strongly modular norm which can be extended to a modular norm on $E^{\prime \prime} \otimes F \supset E \otimes F$, where $E^{\prime \prime}$ is the second dual space of $E$. The work in $\S 5$, which we will discuss presently, is devoted to removing these restrictions. If the restrictions themselves appear somewhat artificial, it must be borne in mind that they are valid for the least cross norm $\lambda$ and for the greatest cross norm $\gamma$, as well as for any modular norm $\alpha$, if both $E$ and $F$ are reflexive. Let us denote an element of $E \otimes_{\alpha} F$ by $t$ and an element of $\left(E \otimes_{\alpha} F\right)^{\prime} \subset \mathscr{L}\left(E, F^{\prime}\right)$ by $S$. We now have (Theorem 6.1) a bilinear mapping $\mathrm{t} \square S$ pairing $E \otimes_{\alpha} F$ and $\left(E \otimes_{\alpha} F\right)^{\prime}$, with values in the tensor product $F^{\prime} \otimes_{\gamma} F$; as well as a corresponding pairing (Theorem 6.2) $S \square \mathrm{t}$ of the same spaces, with values in $E \otimes_{\gamma} E^{\prime}$. We call these two pairings the $\square$-products. The basic formulas connecting these products are

$$
\langle\mathrm{t}, S\rangle=\operatorname{tr}(\mathrm{t} \square S)=\operatorname{tr}(S \square \mathfrak{t}) .
$$

The last two expressions on the right of (1.1) are the traces of tensors in the $\gamma$ tensor product, introduced by Grothendieck. The expression $\langle t, S\rangle$ stands, as usual, for the action of the functional $S$ on the tensor $t$. The $\square$-products are continuous with respect to the natural norms of the spaces in question. Their advantage over $\langle t, S\rangle$, is that, being tensor valued, not merely scalar valued, they contain all the information embodied in $\langle\mathrm{t}, S\rangle$, and much more. The details of this will appear in the form of much more powerful results regarding the duality of $E \otimes_{\alpha} F$ than are possible in general duality theory.

The existence of the $\square$-products is known in various forms for various special cases and can be traced at least as far back as the theorem of Schatten [14] to the effect that the product of two Schmidt operators is a trace class operator. The theorem in our present setting has various converses and partial converses. One is that if $\alpha$ satisfies our assumptions, $S \in \mathscr{L}\left(E, F^{\prime}\right)$ and either of the $\square$-products is well defined for every $\mathrm{t} \in E \otimes_{\alpha} F$, then $S$ must, of necessity, belong to $\left(E \otimes_{\alpha} F\right)^{\prime}$. Another (Theorem 6.3) is that if $\alpha$ is a norm of $E \otimes F$, satisfying very mild assumptions, and $\mathrm{t} \square S$ (or $S \square \mathrm{t}$ ) is well defined for every $S \in\left(E \otimes_{\alpha} F\right)^{\prime}$ and $\mathrm{t} \in E \otimes_{\alpha} F$, then $\alpha$ must be modular. We have described the basic results of $\S 6$. They 
are valid in a very general setting and are of a topological nature, because they all depend on the continuity of the $\square$-products. On the other hand our basic concept of modular norms has a purely algebraic definition, and one would expect that many of the theorems would have purely algebraic hypothesis, but significant topological conclusions. This is the case for a more restrictive class of spaces $E$ and $F$. But for a better description of this we need a discussion centering about the concept of kernels.

It is well known that $E \otimes F$ can be interpreted as a subspace of $\mathscr{L}\left(E^{\prime}, F\right)$, the space of all bounded linear transformations of $E^{\prime}$ into $F$. This interpretation persists in the case of $E \otimes_{\lambda} F$, the result of completing $E \otimes F$ with respect to $\lambda$, because $\lambda$ is really the transformation norm. On the other hand this interpretation may fail for any other norm $\alpha$ of $E \otimes F$. The choice of the verb may is intended to indicate the existence of an almost complete ignorance about this question, rather than the availability of any counterexamples. The ignorance extends even to the case $\alpha=\gamma$, which represents the most famous aspect of this open question. We shall come to this in a moment. Obviously, if $\alpha$ majorizes $\lambda$, then every $t \in E \otimes_{\alpha} F$ defines a bounded linear transformation $T_{\mathrm{t}}: E^{\prime} \rightarrow F$. We follow Grothendieck in calling $\mathrm{t}$ a kernel of $T_{\mathrm{t}}$. The relation between a transformation and its kernel is somewhat analogous to that between an integral transform and its integral kernel, hence the term. The mapping $\mathrm{t} \rightarrow T_{\mathrm{t}}$ is a continuous linear transformation of $E \otimes_{\alpha} F$ into $\mathscr{L}\left(E^{\prime}, F\right)$. However, it may not be isomorphic, i.e., $T_{\mathrm{t}}$ may be 0 for $\mathbf{t} \neq 0$. Given $\alpha$, we shall say that it is faithful if the mapping is isomorphic. Grothendieck was first to realize [11, Chapter I, §5], that $\gamma$ may not be faithful. We shall say that a Banach space $E$ satisfies the condition of approximation of Grothendieck if $\gamma$ is faithful on $E^{\prime} \otimes E$. Grothendieck showed that $E$ satisfies the condition of approximation if and only if every completely continuous linear transformation into $E$ can be uniformly approximated by bounded linear transformations of finite rank. We can now state one of the algebraic characterizations of the individual elements of $\left(E \otimes_{\alpha} F\right)^{\prime}$, if $\alpha$ is a norm satisfying the basic assumptions specified in the previous paragraph. The product $t \square S$ can be easily defined by purely algebraic means, for a tensor $\mathrm{t}$ of the algebraic tensor product $E \otimes F$ and $S \in \mathscr{L}\left(E, F^{\prime}\right)$. Suppose now that $F$ satisfies the condition of approximation of Grothendieck, and $S \in \mathscr{L}\left(E, F^{\prime}\right)$. Then (Theorem 6.7) $S$ belongs to $\left(E \otimes_{\alpha} F\right)^{\prime}$ if and only if the linear transformation $\mathrm{t} \rightarrow \mathrm{t} \square S$ of $E \otimes F$ into $F^{\prime} \otimes F$ can be extended to a (a priori, not known to be bounded) linear transformation $\mathrm{t} \rightarrow \mathrm{t} \square S$ of $E \otimes_{\alpha} F$ into $F^{\prime} \otimes_{\gamma} F$, in such a way that, for every $\mathrm{t} \in E \otimes_{\alpha} F, \mathfrak{t} \square S$ is a kernel of $T_{\mathrm{t}} S^{\prime}$.

The theory described above centering about the $\square$-products is actually developed in the present work for arbitrary modular norms, without the special assumptions that we have been making. We have emphasized the special case because it is simpler and more intuitive. In the general theory, the main difficulty stems from the fact that the role of $\gamma$ in the above discussion of the $\square$-products 
must be played by a new cross norm $\pi$, which we introduce in $\S 5$. The cross norm $\pi$ shares with $\gamma$ many manipulational properties. For instance, the trace of a tensor $\mathfrak{w} \in F^{\prime} \otimes_{\pi} F$ is well defined, but not defined for arbitrary norms on $F^{\prime} \otimes F$. The norm $\pi$ actually coincides with $\gamma$ in many interesting cases, such as when $F$ is reflexive. The theory follows more or less the same lines described above, but now centers about the two products, $\mathrm{t} \times S \in F^{\prime} \otimes_{\pi} F$ and $S \times \mathrm{t} \in E \otimes_{\pi} E^{\prime}$, for $\mathrm{t} \in E \otimes_{\alpha} F$ and $S \in\left(E \otimes_{\alpha} F\right)^{\prime}$.

Once the basic theory has been disposed of, we proceed to the study of tensor products as Banach algebras. Such algebras occur here in two very different forms. One is the tensor product $A \otimes_{\bar{\alpha}} B$ of two Banach algebras $A$ and $B$. The multiplication of elements of $A \otimes_{\bar{\alpha}} B$ is defined by extending distributively the product

$$
\left(U_{1} \otimes V_{1}\right)\left(U_{2} \otimes V_{2}\right)=U_{1} U_{2} \otimes V_{1} V_{2}
$$

of decomposable tensors, for $U_{1}, U_{2} \in A$ and $V_{1}, V_{2} \in B$. We note that $A \otimes_{\bar{\alpha}} B$ is commutative if $A$ and $B$ are commutative. The other algebra is the tensor product $E^{\prime} \otimes_{\alpha} E$, where $E$ is merely a Banach space and $E^{\prime}$ is its dual space. The multiplication in $E^{\prime} \otimes_{\alpha} E$ is defined, for decomposable tensors, by

$$
\left(x^{\prime} \otimes x\right)\left(u^{\prime} \otimes u\right)=\left\langle u, x^{\prime}\right\rangle u^{\prime} \otimes x,
$$

for $x, u \in E$ and $x^{\prime}, u^{\prime} \in E^{\prime}$. We call the algebra $E^{\prime} \otimes_{\alpha} E$ with the multiplication (1.3) an algebra of kernels. We observe that the algebra $E^{\prime} \otimes_{\alpha} E$ is never commutative, unless $E$ is of dimension 1 . These two types of algebras are obviously quite foreign to one another. The multiplication (1.2) is related (see [4]) to multiplication of vector valued functions, pointwise or by convolution. The multiplication (1.3) is related to composition of operators. The two types of algebras have two major features in common. One is that the natural instrument of their study is a modular norm. The other is that the radical in each case (at least when $A$ and $B$ are commutative and semisimple) is the null space of the mapping $\mathrm{t} \rightarrow T_{\mathrm{t}}$ described above. This last fact is somewhat remarkable in view of the completely different nature of the products involved.

The basic theory of the tensor product $A \otimes_{\bar{\alpha}} B$ of Banach algebras was developed by the author in [7] and [10] as an extension to uniform cross norms $\alpha$ of the work of Gelbaum [4], [5] and Tomiyama [15] on $A \otimes_{\gamma} B$. In the present work (\$7) we finally formulate the theory in its most natural setting. We begin by showing (Theorem 7.1) that every modular norm $\alpha$ on $E \otimes F$ is equivalent to a uniform norm on $E \otimes F$, which is a cross norm if $\alpha$ is a cross norm. We use this to reduce the theory of modular cross norms on $A \otimes B$ to that of uniform norms, and apply our methods of [10]. We show here (Theorem 7.3) that every modular norm $\alpha$ on $A \otimes B$ induces on $A \otimes B$ a norm $\bar{\alpha}$ which is compatible with the multiplication (1.2), i.e.,

$$
\bar{\alpha}\left(\mathfrak{I}_{1} \mathfrak{I}_{2}\right) \leqq \bar{\alpha}(\mathfrak{I}) \bar{\alpha}(\mathfrak{T}),
$$


for every $\mathfrak{T}_{1}$ and $\mathfrak{I}_{2} \in A \otimes B$. Thus $A \otimes_{\bar{\alpha}} B$ is a Banach algebra. The rest is an extension of the Gelbaum-Tomiyama theory of the space of maximal ideals. A major achievement of the present approach is that the assumption that $\alpha$ is ordinary, or even a cross norm, employed systematically in [10] has been shown to be entirely superfluous.

The development here ( $\$ 8)$ of the theory of algebras of kernels seems to be entirely new. We begin by showing (Theorem 8.1 ) that every modular norm $\alpha$ of $E^{\prime} \otimes E$ is equivalent to a modular norm of $E^{\prime} \otimes E$ which is compatible with the multiplication (1.3) of kernels. Thus we may assume that $\alpha$ is itself such a norm. Then we show (Theorem 8.2) that the Jacobson radical of $E^{\prime} \otimes_{\alpha} E$ is the null space $\mathscr{N}_{\alpha}$ of the mapping $\mathfrak{v} \rightarrow V_{\mathfrak{v}}$, where $\mathfrak{v} \in E^{\prime} \otimes_{\alpha} E$ and a kernel of $V_{\mathfrak{b}}$. This really follows easily from a theorem of Rickart. We then proceed to show (same Theorem 8.2) that $\mathscr{N}_{\alpha}$ is the left annihilator as well as the right annihilator of the entire algebra $E^{\prime} \otimes_{\alpha} E$, that (Theorem 8.4) it is the only maximal two-sided ideal of $E^{\prime} \otimes_{\alpha} E$ and that it contains all proper two-sided ideals. This yields (Theorem 8.5 ) a complete description of all the two-sided ideals as consisting of all vector subspaces of $\mathscr{N}_{\alpha}$. It also yields (Theorem 8.6) a complete description of all strictly irreducible representations of $E^{\prime} \otimes_{\alpha} E$ as an algebra of operators.

In $\S 9$ we study the duality of the algebra $E^{\prime} \otimes_{\alpha} E$. To simplify the present discussion we assume that the modular norm $\alpha$ satisfies the conditions demanded in (1.1) in connection with the definition of the $\square$-products. The basic result (Theorem 9.1) is that the dual space $\left(E^{\prime} \otimes_{\alpha} E\right)^{\prime}$ is closed under composition of operators, hence is an algebra of operators, and that the dual norm $\alpha^{\prime}$ is equivalent to a norm on $\left(E^{\prime} \otimes_{\alpha} E\right)^{\prime}$ which is compatible with composition of operators. Hence $\left(E^{\prime} \otimes_{\alpha} E\right)^{\prime}$ is an algebra of operators which is a Banach algebra under some norm which majorizes the operator norm and is equivalent to the dual norm $\alpha^{\prime}$. Thus we have in $E^{\prime} \otimes_{\alpha} E$ an instance, though by no means the only one, of the phenomenon of a Banach algebra, whose dual space is also a Banach algebra. But in this case, we have more. We have that the $\square$-products establish a complete pairing between the closed ideals of $E^{\prime} \otimes_{\alpha} E$ and the weakly* closed ideals of $\left(E^{\prime} \otimes_{\alpha} E\right)^{\prime}$. A typical result is (Theorem 9.5) that the annihilator of a left ideal of $E^{\prime} \otimes_{\alpha} E$ under the duality form $\langle\mathfrak{v}, T\rangle, \mathfrak{v} \in E^{\prime} \otimes_{\alpha} E$ and $T \in\left(E^{\prime} \otimes_{\alpha} E\right)^{\prime}$, is its right annihilator under the product $\mathfrak{v} \square T$, hence is a weakly* closed right ideal of $\left(E^{\prime} \otimes_{\alpha} E\right)^{\prime}$. We get the corresponding dual result for right ideals of $\left(E^{\prime} \otimes_{\alpha} E\right)^{\prime}$, as well as a similar pairing under the product $T \square \mathfrak{v}$ of right ideals of $E^{\prime} \otimes_{\alpha} E$ and left ideals of $\left(E^{\prime} \otimes_{\alpha} E\right)^{\prime}$. This yields the result that the weak* closure of an ideal of $\left(E^{\prime} \otimes_{\alpha} E\right)^{\prime}$ is an ideal on the same side. It also yields the result (Theorem 9.8), in view of the work in $\S 8$, that a weakly* closed two-sided ideal of $\left(E^{\prime} \otimes_{\alpha} E\right)^{\prime}$ must contain the annihilator, under $\langle\mathfrak{v}, T\rangle$, of $\mathscr{N}_{\alpha}$ or be trivial. We also get relations (Theorems 9.9 and 9.10) between the topological simplicity of $E^{\prime} \otimes_{\alpha} E$ and that of $\left(E^{\prime} \otimes_{\alpha} E\right)^{\prime}$. The advantage of the availability of the $\square$-products is best illustrated by comparing the algebras $E^{\prime} \otimes_{\alpha} E$ and $\left(E^{\prime} \otimes_{\alpha} E\right)^{\prime}$ with other instances 
of a Banach algebra, whose dual space is a Banach algebra, such as the group algebra $L_{1}(G)$ of a locally compact group $G$, and $L_{\infty}(G)$. Here we see that the daulity form $\langle f, g\rangle, f \in L_{1}(G), g \in L_{\infty}(G)$ is not enough to relate the algebra structure of $L_{1}(G)$ to that of $L_{\infty}(G)$.

In $\$ 10$ we give a few simple concrete examples to illustrate our theory.

As is often the case with a work of the present size, the end product has been made possible by many outside benevolent influences. We are grateful to the National Science Foundation, with whose support the work was actually conceived and executed, and to the Office of Naval Research, who made it possible for the author to come in contact with the Yale school of analysts as an ONR Research Associate. On the personal side the author is grateful to his colleagues B. R. Gelbaum (who is responsible for the author's interest in topological tensor products) and J. T. Joichi. They both contributed greatly to the author's understanding of some of the problems discussed here, to the extent of suggesting methods and improved results. Specific credit is given for these improvements as they occur. The author is equally indebted to Professors E. Hille, S. Kakutani and C. E. Rickart for their kind encouragement and for many stimulating discussions of Banach spaces and Banach algebras. The influence of the book [13] of Rickart on the present work should be plain to anyone who reads both.

2. Modules over Banach algebras. We are going to assume throughout that we are dealing with complex spaces and all terms such as vector space, algebra and the like should be interpreted in the context of the complex field of scalars. Although a good portion of the exposition is valid for real spaces, the setting in terms of complex spaces appears more natural. $E$ and $F$ shall always denote vector spaces. The dual space of a normed space $E$ will be denoted by $E^{\prime}$. For $x \in E$ and $x^{\prime} \in E^{\prime},\left\langle x, x^{\prime}\right\rangle$ will denote the action of $x$ and $x^{\prime}$ on each other. If $F$ is a normed space, the space of all bounded linear transformations $T: E \rightarrow F$ will be denoted by $\mathscr{L}(E, F)$, but the shorter notation $\mathscr{L}(E)$ will be used for the algebra $\mathscr{L}(E, E)$. If $E$ and $F$ are Banach spaces, $\mathscr{L}(E, F)$ is a Banach space under the transformationbound norm $\|T\|$, which turns $\mathscr{L}(E)$ into a Banach algebra.

The first main purpose of the present section is to prove a theorem on the preservation of continuity of the operations on a topological module over a Banach algebra under a change of norm. For this we need a lemma. We let $E$ be for the moment a vector space and \|\|$_{1}$ and \|\|$_{2}$ be two norms on $E$. We shall say that \|\|$_{1}$ is bounded with respect to \|\|$_{2}$ and that \|\|$_{2}$ majorizes $\left({ }^{3}\right)\|\|_{1}$ if there exists a $k>0$ such that $\|x\|_{1} \leqq k\|x\|_{2}$ for every $x \in E$. The following lemma was stated and proved in [8]. We repeat it here (without proof) because it is fundamental in much of what follows.

LEMMA 2.1. Let $E$ be a Banach space and $F$ a normed space under a norm \|\|$_{1}$. Suppose that another norm \|\|$_{2}$ is defined on $F$, under which $F$ is complete and

$\left({ }^{3}\right)$ Term due to Rickart [13]. 
which majorizes \|\|$_{1}$. Assume further that $T: E \rightarrow F$ is a linear transformation which is bounded with respect to \|\|$_{1}$ and the norm of $E$. Then $T$ is bounded with respect to \|\|$_{2}$ and the norm of $E$.

For the proof of Lemma 2.1 we refer the reader to [8]. Its first application will occur shortly.

Let $A$ be a Banach algebra. We follow the practice of [7] and [10] of designating the elements of $A$ by symbols such as $U$, which suggest linear operators. Let $M$ be a complex vector space. We shall say that $M$ is a left module over $A$ if there is a bilinear mapping $(U, x) \rightarrow U x, U \in A, x \in M$, of $A \times M$ into $M$ such that $U(\zeta x)=(\zeta U) x=\zeta U x$, for every complex scalar $\zeta$. A right module over $A$ is defined analogously. If $M$ is both a right and a left module over $A$, we say that $M$ is a two-sided module over $A$. A submodule (left, right, two-sided) of $M$ is a vector subspace of $M$, which remains invariant under the appropriate (left, right, twosided) action of $A$. Let $M$ be a normed space and a left (right) module over $A$. We say that $M$ is a topological left (right) module over $A$ if the defining bilinear mapping is continuous simultaneously in both variables. It is well known, and easy to see, that this continuity of the bilinear mapping is equivalent to its boundedness. For example, for left modules this means that there exists a $k>0$ such that

$$
\|U x\| \leqq k\|U\|\|x\|,
$$

for every $U \in A$ and $x \in M$. It is also well known, and follows directly from the uniform boundedness theorem and from (2.1), that if $M$ is complete it is sufficient that $U x$ be continuous separately in $U$ and $x$ in order for it to be jointly continuous. Of course, a similar statement holds for right modules. We are now ready to state the main result of this section.

THEOREM 2.1. Let $M$ be a left (right) module over a Banach algebra $A$ and \|\|$_{1}$ a norm of $M$ which is bounded with respect to a second norm \|\|$_{2}$ of $M$. Suppose that $M$ is complete under \|\|$_{2}$ and a topological left (right) module over $A$, with respect to \|\|$_{1}$. Then $M$ is a topological left (right) module over $A$ with respect to \|\|$_{2}$.

Proof (for left modules). According to the remarks preceding this theorem, it suffices to show that $U x$ is separately continuous in $U$ and $x$ with respect to $\|\quad\|_{2}$ and the norm of $A$, which remains in the background and which we do not mention unless it is necessary. Let $x$ be a fixed element of $M$. Then the mapping $U \rightarrow U x$ of $A$ into $M$ is continuous with respect to \|\|$_{1}$ and, by Lemma 2.1, continuous with respect to \|\|$_{2}$. Hence $U x$ is continuous in $U$ with respect to \|\|$_{2}$, for fixed $x \in M$. Let now $U$ be a fixed element of $A$. Now the mapping $x \rightarrow U x$ of $M$ into itself is continuous with respect to \|\|$_{1}$ as the norm of the domain and with respect to \|\|$_{1}$ as the norm of the range. Since \|\|$_{1}$ is bounded with respect to \|\|$_{2}$, we may replace \|\|$_{1}$ by \|\|$_{2}$ on the domain and preserve 
continuity. We again apply Lemma 2.1 , using for $E$ in that lemma the present space $M$ with $\|\quad\|_{2}$ and for $F$ the present space $M$ with \|\|$_{1}$. We conclude from Lemma 2.1 that $x \rightarrow U x$ is continuous with respect to \|\|$_{2}$ both as the range and domain norm. Thus we have shown that $U x$ is continuous, separately in each variable. Hence it is jointly continuous. Q.E.D.

The following theorem is but a trivial consequence of Theorem 2.1 , and its discussion is omitted. It is stated separately because its form is the most convenient for later reference.

THEOREM 2.2. Let $M$ be a topological left module over a Banach algebra $A$ and $a$ topological right module over a Banach algebra $B$, with respect to a norm \|\|$_{1}$ of $M$. Suppose that \|\|$_{2}$ is a second norm of $M$ which majorizes \|\|$_{1}$, and with respect to which $M$ is complete. Then there exists a constant $k>0$ such that, for every $x \in M, U \in A$ and $V \in B$, we have $\left({ }^{4}\right)$

$$
\begin{aligned}
\|U x\|_{2} & \leqq k\|U\|\|x\|_{2}, \\
\|x V\|_{2} & \leqq k\|V\|\|x\|_{2}, \\
\|U x V\|_{2} & \leqq k\|U\|\|V\|\|x\|_{2} .
\end{aligned}
$$

The next theorem is an application of Theorem 2.2. It provides a method for establishing the topological simplicity of a Banach algebra by imbedding it continuously and densely in a topologically simple Banach algebra, such as a topologically simple algebra of operators. We follow Rickart [13, p. 101] in saying that a Banach algebra is topologically simple if it contains no closed two-sided ideals other than $\{0\}$ and the entire algebra.

THEOREM 2.3. Let $A$ be a topologically simple Banach algebra with respect to a norm \|\|$_{1}$. Let $B$ be a dense two-sided ideal of $A$, which is, itself, a Banach algebra with respect to a second norm \|\|$_{2}$, which majorizes \|\|$_{1}$ on $B$. Then $B$ is topologically simple if and only if one of the following three conditions holds:

(a) $B$ is one-dimensional,

(b) $B$ is zero-dimensional,

(c) The vector $\left({ }^{5}\right)$ space generated by $B^{2}$ (i.e., the set of all finite sums of products $X Y$, for $X, Y \in B)$ is dense in $B$ with respect to \|\|$_{2}$.

Proof. Suppose that $B$ is topologically simple. The vector space generated by $B^{2}$ is a two-sided ideal of $B$, which is either $\{0\}$ or dense in $B$. If it is $\{0\}$, it follows from the topological simplicity of $B$ that $B$ cannot have any proper vector subspace except 0 , hence it must be one-dimensional, or zero-dimensional.

Suppose conversely that (a) or (b) is satisfied. Then $B$ is obviously topologically simple. Suppose that (c) is satisfied. We show that $B$ is topologically simple.

${ }^{(4)}\|\quad\|$ stands for the only norm on $A$ under consideration.

(5) We are indebted to B. Natzitz for a suggestion which leads to this condition, a considerable improvement over an earlier version. 
Let $U$ and $V$ represent general elements of $A$ and $W$ the general element of $B$. Then, since $B$ is a two-sided ideal of $A$, the bilinear expressions $U W$ and $W V$ define $B$ as a left and right module over $A$. It is a topological module if we use the norm \|\|$_{1}$ on both $A$ and $B$. We can now apply Theorem 2.2 with $\|$ \|replaced by \|\|$_{1}$ in the present algebra $A$. We conclude that (2.2) through (2.4) are valid with $x$ replaced by $W$. This fact will be used later on in the proof.

Let $C$ be a two-sided ideal of $B$ different from $\{0\}$. The object is to show that $C$ is dense in $B$ with respect to \|\|$_{2}$. Let $\bar{C}$ denote the closure of $C$ with respect to $\|\quad\|_{2}$ and $\widetilde{C}$ the closure of $C$ in $A$ with respect to \|\|$_{1}$. We shall show that $\widetilde{C}$ is a two-sided ideal of $A$. Let $U \in A$ and $Y \in \mathcal{C}$. Suppose $Y_{1}, Y_{2}, \cdots$ is a sequence in $C$ converging to $Y$ with respect to \|\|$_{1}$ and $U_{1}, U_{2}, \cdots$ a sequence of elements of $B$ converging to $U$ with respect to \|\|$_{1}$. Then $U_{n} Y_{n} \in C$ and $U Y \in \widetilde{C}$. This shows that $\tilde{C}$ is a left ideal. A similar argument shows that $\widetilde{C}$ is a right ideal. Since $A$ is topologically simple, we have $\widetilde{C}=A$. We now show that $C$ is dense in $B$ with respect to \|\|$_{2}$. We use the assumption that the vector space generated by $B^{2}$ is dense in $B$. Let $S \in B$. Then there exists a sequence $\left\{\sum_{i=1}^{k_{n}} X_{i}^{n} Y_{i}^{n}\right\}_{n}, X_{i}^{n}$, $Y_{i}^{n} \in B$, such that $\left\|S-\sum_{i=1}^{k_{n}} X_{i}^{n} Y_{i}^{n}\right\|_{2} \rightarrow 0$ as $n \rightarrow \infty$. Now, since $C$ is dense in $A$ with respect to \|\|$_{1}$, there exist elements $W_{i}^{n}$ of $C$ such that

$$
\sum_{i=1}^{k_{n}}\left\|X_{i}^{n}\right\|_{2}\left\|Y_{i}^{n}-W_{i}^{n}\right\|_{1} \rightarrow 0 \quad \text { as } n \rightarrow \infty .
$$

We now apply Theorem 2.2 with the appropriate substitutions, to get

$$
\begin{aligned}
& \left\|S-\sum_{i=1}^{k_{n}} X_{i}^{n} W_{i}^{n}\right\|_{2} \leqq\left\|S-\sum_{i=1}^{k_{n}} X_{i}^{n} Y_{i}^{n}\right\|_{2}+\left\|\sum_{i=1}^{k_{n}} X_{i}^{n}\left(Y_{i}^{n}-W_{i}^{n}\right)\right\|_{2} \\
& \leqq\left\|S-\sum_{i=1}^{k_{n}} X_{i}^{n} Y_{i}^{n}\right\|_{2}+k \sum_{i=1}^{k_{n}}\left\|X_{i}^{n}\right\|_{2}\left\|Y_{i}^{n}-W_{i}^{n}\right\|_{1} .
\end{aligned}
$$

The last expression in (2.5) tends to 0 as $n \rightarrow \infty$. Since each $X_{i}^{n} W_{i}^{n} \in C$, this shows that $C$ is dense in $B$ with respect to \|\|$_{2}$, hence that $B$ is topologically simple.

The next theorem is a converse of Theorem 2.3.

THEOREM 2.4. Let $A$ be a Banach algebra with respect to a norm \|\|$_{1}$, and $B$ a dense two-sided ideal of $A$ which is itself a topologically simple Banach algebra with respect to a second norm \|\|$_{2}$ which majorizes \|\|$_{1}$ on $B$. Then $A$ is topologically simple with respect to \|\|$_{1}$ if and only if one of the following three conditions is satisfied: (a) $A$ has no left annihilator other than 0 , (b) $A$ has no right annihilator other than 0, (c) $A$ is one-dimensional.

Proof. Suppose that $A$ is topologically simple. Then both the set of left annihilators and the set of right annihilators of $A$ are two-sided ideals, which either reduce to $\{0\}$ or force $A$ to be one-dimensional. 
Conversely, suppose that one of the three conditions is satisfied. If (c) is satisfied, then $A$ is obviously topologically simple. Suppose that either (a) or (b) is satisfied. The reasoning that will follow applies equally well to (a) as to (b). Consequently, to fix our ideas we assume that it is (a) that is satisfied. We then show that $A$ is topologically simple. This amounts to showing that every two-sided ideal $C$ of $A$, which contains elements $\neq 0$, is dense in $A$ with respect to \|\|$_{1}$. Since (a) is satisfied, $C B$, the set of all products $W S, W \in C, S \in B$, has nonzero elements, otherwise, $B$ being dense in $A, C A$ would reduce to $\{0\}$, violating (a). The vector space $L$ generated by $C B$ is a two-sided ideal of $A$ contained in $B \cap C$, hence must be dense in $B$ with respect to \|\|$_{2}$, since $B$ is topologically simple, hence must be dense in $A$. Then $C$, which contains $L$ must also be dense in $A$. Q.E.D.

We are now in a position to prove a representation theorem for topologically simple Banach algebras, which characterizes them as algebras of operators.

THEOREM 2.5. Let $B$ be a Banach algebra of dimension $\neq 0,1$. Then $B$ is topologically simple if and only if the vector space generated by $B^{2}$ is dense in $B$ and there exists a faithful continuous representation of $B$ as a dense two-sided ideal of a topologically simple Banach algebra of bounded operators, the latter algebra with the operator norm.

Proof. First let us suppose that $B$ is a topologically simple Banach algebra. Then clearly, the vector space generated by $B^{2}$, which is a two-sided ideal, is dense in $B$. We now represent $B$ as an algebra of operators on a Banach space $E$. We let $E$ be $B$ itself considered as a Banach space, disregarding its multiplicative structure. Let us map $B$ into $\mathscr{L}(E)$ by means of the left regular representation. It is well known that this representation is continuous. Let us show that it is isomorphic. If not, the set of all left annihilators of $B$, a two-sided ideal would be the entire $B$, by the topological simplicity of $B$. This would make $B$ one- or zero-dimensional. Hence the representation is isomorphic. We now let $A$ be the closure of the image of $B$ in $\mathscr{L}(E)$. We shall conclude the proof by showing that $A$ is topologically simple and that $B$ is a two-sided ideal of $A$.

Let us show that $B$ is a two-sided ideal of $A$. First, it is a left ideal, for if $T \in A$ and $U \in B$, the expression $T U$ can be interpreted both as the product of $T$ and $U$ in $A$ and as the image of $U \in E$ under the operator $T$. The latter interpretation yields $T U \in B$. Hence $B$ is a left ideal.

The proof that $B$ is a right ideal is slightly more difficult. Let $U$ and $V$ be elements of $B$. Denote the given norm of $B$ by \|\|$_{2}$ and the operator norm in $\mathscr{L}(E)$ by $\|\quad\|$, as usual. Since $B$ is a subalgebra of $A$ we have

$$
\|U V\| \leqq\|U\|\|V\| \text {. }
$$

Now let $V$ be, for the moment, a fixed element of $B$. The mapping $U \rightarrow U V$ represents a bounded operator of the Banach space $E=B$ with \|\|$_{2}$. Also, by (2.6), as $V$ varies over the $\|\quad\|$-unit sphere of $B$ the mappings $U \rightarrow U V$ are bounded 
( $U$ fixed in $B$ ). By the uniform boundedness theorem we conclude that they are uniformly bounded. This means that there exists a $k^{\prime}>0$ such that

$$
\|U V\|_{2} \leqq k^{\prime}\|U\|_{2}\|V\| \text {. }
$$

Now we are in a position to prove that $B$ is a right ideal of $A$. Let $U \in B$ and $T \in A$. We have to show that $U T \in B$. Since $B$ is dense in $A$ there is a sequence $V_{1}, V_{2}, \cdots$ of elements of $B$ converging to $T$ with respect to \|\| . Hence that sequence is a Cauchy sequence with respect to \|\| . It follows from (2.7) that the sequence $U V_{1}, U V_{2}, \cdots$ is a Cauchy sequence with respect to \|\|$_{2}$, hence converges to an element of $B$ because $B$ is complete with respect to \|\|$_{2}$. The limit element must be $U T$. This shows that $B$ is a right, hence a two-sided ideal of $A$. It follows now from Theorem 2.4 that $A$ is topologically simple.

Conversely, let us assume that the vector space generated by $B^{2}$ is dense in $B$ and that there exists the required faithful representation. Then we may assume that $B$ itself is a dense two-sided ideal of a topologically simple Banach algebra of operators. Theorem 2.3 implies that $B$ is topologically simple. This completes the proof of our theorem.

3. Tensor products of Banach spaces. In this section we summarize some of the known definitions and results from the work of Schatten [14] and Grothendieck [11] on the theory of topological tensor products, in a setting most appropriate to our present work. Our approach is considerably less general than that of Grothendieck but somewhat more general than Schatten's. With the latter we study the tensor product $E \otimes F$ of two Banach spaces $E$ and $F$ and a norm $\alpha$ on it. By contrast $\alpha$ need not be a cross norm, but shares with cross norms most of the essential topological properties. The reason for our present departure from the approach of Schatten and our own in previous works [6], [7], [8], [9], and [10] is that some of the important processes described in $\$ \S 8$ and 9 below, may destroy the metric character of cross norms, but do preserve their topological properties.

A tensor $\mathrm{t}$ of the algebraic tensor product $E \otimes F$ can be identified with a bounded linear transformation $T_{\mathrm{t}}: E^{\prime} \rightarrow F$ of finite rank. If $\mathrm{t}$ is represented by

$$
\mathrm{t}=\sum_{i=1}^{n} x_{i} \otimes y_{i}
$$

for $x_{i} \in E$ and $y_{i} \in F$, then $T_{\mathrm{t}}$ is given, for $x^{\prime} \in E^{\prime}$ by

$$
T_{\mathrm{t}} x^{\prime}=\sum_{i=1}^{n}\left\langle x_{i}, x^{\prime}\right\rangle y_{i}
$$

Although the mapping $\mathrm{t} \rightarrow T_{\mathrm{t}}$ is an algebraic isomorphism of the algebraic tensor product $E \otimes F$ into $\mathscr{L}\left(E^{\prime}, F\right)$, we shall in general differentiate between a tensor $t$ and the transformation $T_{\mathrm{t}}$ which it defines. This is because we shall soon be discussing the completion of $E \otimes F$ under various norms, and for tensors $t$ in the resulting complete tensor products, the extended mapping $t \rightarrow T_{\mathfrak{t}}$ (if it exists) is 
not, in general, known to be isomorphic. Schatten [14], defined a norm $\alpha$ on $E \otimes F$ to be a cross norm if $\alpha(x \otimes y)=\|x\|\|y\|$ for every $x \in E$ and $y \in F$. He introduced two particular cross norms that are of great importance in the theory. One is the norm $\lambda$, defined, for $\mathrm{t} \in E \otimes F$ by

$$
\lambda(\mathrm{t})=\left\|T_{\mathrm{t}}\right\|,
$$

where $\left\|T_{\mathrm{t}}\right\|$ is the transformation norm. The norm $\lambda$ is referred to as the least cross norm. The second cross norm is the greatest cross norm $\gamma$ given by the formula

$$
\gamma(\mathrm{t})=\inf \sum_{i=1}^{n}\left\|x_{i}\right\|\left\|y_{i}\right\|
$$

where the inf is taken over all possible representations of $t$ of the form (3.1).

It will be necessary for us to consider norms on the tensor products $E \otimes F$ which are more general than cross norms. A norm $\alpha$ on $E \otimes F$ is said to be tensorial if it is majorized (see $\$ 2$ ) by the greatest cross norm $\gamma$. We give here a summary of the essential parts of the theory of Schatten-Grothendieck in terms of the new concept of tensorial norm. The proofs for cross norms can be found in [14] and/or [11], and the modifications necessary to allow for the present setting are inessential. Clearly, all cross norms, in particular $\lambda$ and $\gamma$, are tensorial. Let $\alpha$ be a tensorial norm of $E \otimes F$. We denote by $E \otimes_{\alpha} F$ the completion of $E \otimes F$ under the norm $\alpha$, and refer to this complete space as the $\alpha$-tensor product of $E$ and $F$.

It should be noticed that, in view of (3.3) the least-cross norm tensor product $E \otimes_{\lambda} F$ can be isometrically imbedded into the Banach space $\mathscr{L}\left(E^{\prime}, F\right)$ of all bounded linear transformations of $E^{\prime}$ into $F$, with the transformation norm. Let us denote this imbedding by $\Psi^{\lambda}: E \otimes_{\lambda} F \rightarrow \mathscr{L}\left(E^{\prime}, F\right)$ and by $T_{\mathrm{t}}$ the transformation $\Psi^{\lambda} \mathrm{t}$, given $\mathrm{t} \in E \otimes_{\lambda} F$.

Let now $\alpha$ and $\beta$ be two tensorial norms of $E \otimes F$ such that $\alpha$ majorizes $\beta$. In this case the identity mapping of the dense subspace $E \otimes F$ of $E \otimes_{\alpha} F$ into $E \otimes F$ can be extended to a bounded linear transformation $\Phi_{\beta}^{\alpha}: E \otimes_{\alpha} F \rightarrow E \otimes_{\beta} F$.

We shall say that a tensorial norm $\alpha$ of $E \otimes F$ is admissible (term from [3]) if $\alpha$ majorizes $\lambda$. This concept is the form which the concept, described by Grothendieck $[11$, p. 88$]$ by the phrase compatible with the tensor product structure, assumes for normed spaces. It is also the topological equivalent of the metric concept of ordinary cross norm, used by the present author in [7] and [10]. $\alpha$ is said to be ordinary if

$$
\lambda \leqq \alpha \leqq \gamma
$$

Clearly an ordinary norm is admissible and, of necessity, a cross norm. We see from the previous paragraph that for an admissible norm, the mapping $\Phi_{\lambda}^{\alpha}: E \otimes_{\alpha} F \rightarrow E \otimes_{\lambda} F$ is well defined. We define $\Psi^{\alpha}: E \otimes_{\alpha} F \rightarrow \mathscr{L}\left(E^{\prime}, F\right)$ by

$$
\Psi^{\alpha}=\Psi^{\lambda} \Phi_{\lambda}^{\alpha} .
$$


We call $\Psi^{\alpha}$ the natural mapping of $E \otimes_{\alpha} F$ into $\mathscr{L}\left(E^{\prime}, F\right)$. For $\mathrm{t} \in E \otimes_{\alpha} F$, we define $T_{\mathrm{t}}: E^{\prime} \rightarrow F$ by

$$
T_{\mathfrak{t}}=\Psi^{\alpha} \mathrm{t}
$$

Following Grothendieck [11, p. 80] we refer to $t$ as a kernel of $T_{\mathrm{t}}$. We sometimes refer to a tensor $\mathrm{t}$ of $E \otimes_{\alpha} F$ as a kernel of type $\alpha$. The transformation $T_{\mathrm{t}}$ is always compact and continuous with respect to the weak* topology of $E^{\prime}$ and the weak topology of $F[11]$.

One striking contrast between the natural mapping for $\lambda$ and that for a general admissible $\alpha$ is that, while the former is isomorphic (indeed isometric), the latter is not known, in general, to be isomorphic. In [10] we have used the term faithful to describe an ordinary norm $\alpha$ for which the natural mapping of $E \otimes_{\alpha} F$ into $\mathscr{L}\left(E^{\prime}, F\right)$ is isomorphic and we shall use the same term here in connection with admissible norms. As we have stated in the introduction, the faithful character of the greatest cross norm $\gamma$ is related to the problem of approximating compact operators uniformly by operators of finite rank. Thus we see that it is not possible to identify readily a tensor $\mathrm{t} \in E \otimes_{\alpha} F$ with the transformation $T_{\mathrm{t}}$.

Although a tensor is not readily identified with a linear transformation, an element of the dual space $\left(E \otimes_{\alpha} F\right)^{\prime}$ can always be identified with a bounded linear transformation $S: E \rightarrow F^{\prime}$, regardless of whether $\alpha$ majorizes $\lambda$ or not, provided that $\alpha$ is tensorial. This fact was first discovered by Schatten [14]. Since much of what follows in this article depends on it, we discuss the situation in some detail. We follow the approach of Grothendieck [11]. We shall always denote the transpose (adjoint) of a bounded linear transformation $W: E \rightarrow F$ by the symbol $W^{\prime}$. By the same token, for $\mathrm{t} \in E \otimes F$ with representation (3.1), we define $\mathrm{t}^{\prime} \in F \otimes E$ by

$$
\mathrm{t}^{\prime}=\sum_{i=1}^{n} y_{i} \otimes x_{i}
$$

Clearly the resulting tensor $t^{\prime}$ is independent of the particular representation (3.1) of $\mathrm{t}$. We call $\mathrm{t}^{\prime}$ the transpose of $\mathrm{t}$. Obviously $T_{\mathrm{t}^{\prime}}=\left(T_{\mathrm{t}}\right)^{\prime}$. Let now $\mathfrak{v} \in E^{\prime} \otimes E$ have a representation

$$
\mathfrak{v}=\sum_{j=1}^{m} x_{j}^{\prime} \otimes x_{j}
$$

We define the trace $\operatorname{tr} \mathfrak{v}$ of $\mathfrak{v}$ as

$$
\operatorname{tr} \mathfrak{v}=\sum_{j=1}^{n}\left\langle x_{j}, x_{j}^{\prime}\right\rangle .
$$

It is easy to see that $\operatorname{tr} \mathfrak{v}$ does not depend on the particular representation (3.9) of $\mathfrak{v}$. It is also easy to verify that if $E$ is finite dimensional, then $\operatorname{tr} \mathfrak{v}$ is the trace of the matrix of the transformation defined by $\mathfrak{v}$. We also have

$$
\operatorname{tr} \mathfrak{v}=\operatorname{tr} \mathfrak{v}^{\prime} .
$$


Let us now return to our tensors $\mathrm{t} \in E \otimes F$ with representation (3.1). Let $S \in \mathscr{L}\left(E, F^{\prime}\right)$. We define the two tensors $S \times \mathrm{t} \in E \otimes E^{\prime}$ and $\mathrm{t} \times S \in F^{\prime} \otimes F$ by the relations

$$
S \times \mathrm{t}=\sum_{i=1}^{n} x_{i} \otimes S^{\prime} y_{i}
$$

and

$$
\mathrm{t} \times S=\sum_{i=1}^{n} S x_{i} \otimes y_{i}
$$

Clearly, $S \times \mathrm{t}$ is a kernel of $S^{\prime} T_{\mathrm{t}}$, and $\mathrm{t} \times S$ is a kernel of $T_{\mathrm{t}} S^{\prime}$. In the following relations, it is easy to verify that the various traces involved are well defined objects, and to check that the relations themselves are valid.

$$
\operatorname{tr} S \times \mathrm{t}=\operatorname{tr} \mathrm{t} \times S=\operatorname{tr} S^{\prime} \times \mathfrak{t}^{\prime}=\operatorname{tr} \mathfrak{t}^{\prime} \times S^{\prime} .
$$

We are now ready to discuss the duality of $E \otimes_{\alpha} F$, for any tensorial norm $\alpha$ of $E \otimes F$. For $\mathrm{t} \in E \otimes F$ and $S \in \mathscr{L}\left(E, F^{\prime}\right)$, define

$$
\langle\mathrm{t}, S\rangle=\operatorname{tr} S \times \mathrm{t} .
$$

The relation (3.15) enables us to consider the elements $S$ of $\mathscr{L}\left(E, F^{\prime}\right)$ as elements of the algebraic dual of $E \otimes F$. It was discovered by Schatten [14] that the topological dual of $E \otimes_{\alpha} F$ can be identified with the space of all such transformations $S$ corresponding to functionals that are bounded with respect to $\alpha$. For such an $S$ the action $\langle\mathrm{t}, S\rangle$ of $S$ on $E \otimes F$ is extended by the usual continuity argument to all of $E \otimes_{\alpha} F$. In $\$ 6$ below we shall be able to give a more explicit expression for $\langle\mathrm{t}, S\rangle$ for any $\mathrm{t} \in E \otimes_{\alpha} F$, in the case of a class of norms $\alpha$ which we call modular. Thus we can (and will) consider $\left(E \otimes_{\alpha} F\right)^{\prime}$ as a vector subspace of $\mathscr{L}\left(E, F^{\prime}\right)$. It is not in general closed with respect to the topology of $\mathscr{L}\left(E, F^{\prime}\right)$, although it is, of course, a Banach space with respect to the dual norm $\alpha^{\prime}$. However, we have the following relation due to Schatten [14, Theorem 3.2, p. 47], for the greatest cross norm $\gamma$,

$$
\left(E \otimes_{\gamma} F\right)^{\prime}=\mathscr{L}\left(E, F^{\prime}\right),
$$

isometrically. In particular, it follows that the Banach algebra $\mathscr{L}\left(E^{\prime}\right)$ considered as a Banach space is the dual space of the tensor product $E^{\prime} \otimes_{\gamma} E$. For this tensor product, it was shown by Grothendieck [11, p. 78], that the trace, defined above in (3.10) for tensors in $E^{\prime} \otimes E$ can be extended to the entire $E^{\prime} \otimes_{\gamma} E$. This can be done in many ways, the simplest, perhaps, being

$$
\operatorname{tr} \mathrm{t}=\langle\mathrm{t}, I\rangle \text {, }
$$

for any $\mathrm{t} \in E^{\prime} \otimes_{\gamma} E$. In (3.17) I stands for the identity operator of $E^{\prime}$.

Consider now the tensor product $E^{\prime} \otimes F^{\prime}$. Under the earlier conventions of this section, this space is imbedded, under the natural mapping, into $\mathscr{L}\left(E^{\prime \prime}, F^{\prime}\right)$. 
However, we are going to effect an imbedding of $E^{\prime} \otimes F^{\prime}$ into $\mathscr{L}\left(E, F^{\prime}\right)$. This is done as follows. Each $\mathfrak{g} \in E^{\prime} \otimes F^{\prime}$ is a kernel of a bounded linear transformation $S_{3}: E^{\prime \prime} \rightarrow F^{\prime}$. But $S_{3}$ is continuous with respect to the weak* topology of $E^{\prime \prime}$ and the weak topology of $F^{\prime}$. Consequently, it is completely determined by its action on $E \subset E^{\prime \prime}$. For our purposes it is convenient, and possible, to identify this transformation with its restriction to $E$, for which we use the same symbol $S_{q}$, whenever there is no possibility of confusion. Hence, under the assignment $\mathfrak{B} \rightarrow S_{\mathfrak{3}}, S_{\mathfrak{3}}: E \rightarrow F^{\prime}$, we imbed $E^{\prime} \otimes F^{\prime}$ into $\mathscr{L}\left(E, F^{\prime}\right)$. We also refer to this imbedding as the natural mapping. This ambiguity will not cause any confusion.

Let now $\alpha$ be a norm of $E \otimes F$ which is not only tensorial but also admissible. Then we have that the natural image of $E^{\prime} \otimes F^{\prime}$ into $\mathscr{L}\left(E, F^{\prime}\right)$, discussed in the preceding paragraph, is actually contained in $\left(E \otimes_{\alpha} F\right)^{\prime} \subset \mathscr{L}\left(E, F^{\prime}\right)$. This is because this fact is easily checked for $\alpha=\lambda$, and must remain valid for any tensorial norm $\alpha$ which majorizes $\lambda$. See [14, p. 32, Theorem 2.1]. For $x^{\prime} \in E^{\prime}, y^{\prime} \in F^{\prime}$ and $\mathfrak{t} \in E \otimes_{\alpha} F$, one can easily verify the relation

$$
\left\langle\mathrm{t}, S_{x^{\prime} \otimes y^{\prime}}\right\rangle=\left\langle T_{\mathrm{t}} x^{\prime}, y^{\prime}\right\rangle .
$$

We shall denote the norm of the dual space $\left(E \otimes_{\alpha} F\right)^{\prime}$ by $\alpha^{\prime}$, and refer to it as the dual norm of $\alpha$. Clearly, under the imbedding of $E^{\prime} \otimes F^{\prime}$ into $\left(E \otimes_{\alpha} F\right)^{\prime}$, the dual norm $\alpha^{\prime}$ induces a norm on $E^{\prime} \otimes F^{\prime}$, which we continue to denote by $\alpha^{\prime}$. One checks easily that $\alpha^{\prime}$ is admissible on $E^{\prime} \otimes F^{\prime}$. Hence, the tensor product $E^{\prime} \otimes_{\alpha^{\prime}} F^{\prime}$ is well defined.

4. Modular norms. Let us now consider the algebra $\mathscr{L}\left(F^{\prime}\right)$. We are going to use the symbol $\mathscr{L}^{\#}\left(F^{\prime}\right)$ to denote the subset of $\mathscr{L}\left(F^{\prime}\right)$ consisting of all those operators $U$ with the property that $U^{\prime}: F^{\prime \prime} \rightarrow F^{\prime \prime}$ maps $F$ into itself. Such an operator defines an operator $U^{\#}: F \rightarrow F$, the restriction of $U^{\prime}$ to $F$. It is well to know (although we know of no specific reference) that an element $U$ of $\mathscr{L}\left(F^{\prime}\right)$ belongs to $\mathscr{L}^{\#}\left(F^{\prime}\right)$ if and only if it is the transpose of an element of $\mathscr{L}(F)$, (namely, $U^{\#}$ ) and if and only if it is a continuous operator on $F^{\prime}$, when the latter is given its weak* topology. Clearly $\mathscr{L}^{\#}\left(F^{\prime}\right)$ is a closed subalgebra of $\mathscr{L}\left(F^{\prime}\right)$. Let us now consider $\mathscr{L}\left(E, F^{\prime}\right)$. This space can be regarded as a left module $(\S 1)$ over the algebra $\mathscr{L}\left(F^{\prime}\right)$. For $U \in \mathscr{L}\left(F^{\prime}\right)$ and $S \in \mathscr{L}\left(E, F^{\prime}\right)$, the composition $U S$ implements the action of $\mathscr{L}\left(F^{\prime}\right)$ on $\mathscr{L}\left(E, F^{\prime}\right)$. In a similar manner we regard $\mathscr{L}\left(E, F^{\prime}\right)$ as a left module over the algebra $\mathscr{L}^{\#}\left(F^{\prime}\right)$. Since we will have occasion to use both structures of left module we shall distinguish between them by refering to $\mathscr{L}\left(E, F^{\prime}\right)$ as an $\mathscr{L}\left(F^{\prime}\right)$-left module or $\mathscr{L}^{\#}\left(F^{\prime}\right)$-left module, according to the context. We follow similar conventions in regard to other notions, such as submodules. Similarly we can regard $\mathscr{L}\left(E, F^{\prime}\right)$ as a right module over $\mathscr{L}(E)$.

It is of interest in connection with the discussion that will follow shortly to realize that the space $\mathscr{L}\left(E, F^{\prime}\right)$ is isometrically isomorphic to $\mathscr{L}\left(F, E^{\prime}\right)$, the isomorphism implemented by the assignment $S \rightarrow S^{\#}$, where $S \in \mathscr{L}\left(E, F^{\prime}\right)$ and $S^{\#}$ 
is the restriction of $S^{\prime}$ to $F$. This mapping is easily shown to be isometric and onto. It is also relatively easy to show that under this mapping an $\mathscr{L}(E)$-right submodule of $\mathscr{L}\left(E, F^{\prime}\right)$ goes onto an $\mathscr{L}^{\#}\left(E^{\prime}\right)$-left submodule of $\mathscr{L}\left(F, E^{\prime}\right)$ and an $\mathscr{L}^{\#}\left(F^{\prime}\right)$-left submodule goes onto an $\mathscr{L}(F)$-right submodule.

Clearly an $\mathscr{L}\left(F^{\prime}\right)$-left submodule of $\mathscr{L}\left(E, F^{\prime}\right)$ is an $\mathscr{L}^{\#}\left(F^{\prime}\right)$-left submodule of $\mathscr{L}\left(E, F^{\prime}\right)$. One verifies easily that the natural image (\$2) of $E^{\prime} \otimes F^{\prime}$ in $\mathscr{L}\left(E, F^{\prime}\right)$ is an $\mathscr{L}\left(F^{\prime}\right)$-left submodule of $\mathscr{L}\left(E, F^{\prime}\right)$ and an $\mathscr{L}(E)$-right submodule. The usefulness of the submodules of $\mathscr{L}\left(E, F^{\prime}\right)$ is based on their property embodied in the following theorem. This theorem seems to be well known by many people, but the author has not seen it in the literature. The proof given here is essentially one suggested orally by B. R. Gelbaum. The argument is also very close to that given in [13, Lemma 2.8 .20$, p. 101] in a somewhat different context.

THEOREM 4.1. Let $\mathscr{M}$ be any vector subspace of $\mathscr{L}\left(E, F^{\prime}\right)$ different from 0 , which is an $\mathscr{L}^{\#}\left(F^{\prime}\right)$-left submodule and an $\mathscr{L}(E)$-right submodule. Then $\mathscr{M}$ contains the natural image of $E^{\prime} \otimes F^{\prime}$ in $\mathscr{L}\left(E, F^{\prime}\right)$.

Proof. It suffices to show that $\mathscr{M}$ contains all bounded linear transformations from $E$ into $F^{\prime}$ of rank one. These correspond to tensors $x^{\prime} \otimes y^{\prime} \in E^{\prime} \otimes F^{\prime}$, for $x^{\prime} \neq 0$ and $y^{\prime} \neq 0$. Fix two such $x^{\prime}$ and $y^{\prime}$. We show $S_{x^{\prime} \otimes y^{\prime}} \in \mathscr{M}$. Let $S_{1}$ be an element of $\mathscr{M}$ different from 0 . The transpose transformation $S_{1}^{\prime}$ maps $F^{\prime \prime}$ into $E^{\prime}$. Let $v \in F \subset F^{\prime \prime}$ be such that $S_{1}^{\prime} v \neq 0$. Let $u_{0} \in E$ be such that $\left\langle u_{0}, S_{1}^{\prime} v\right\rangle=1$. Let us now consider the original $x^{\prime}$, and let $u_{1} \in E$ be such that $\left\langle u_{1}, x^{\prime}\right\rangle=1$. Now $V_{x^{\prime} \otimes u_{0}} \in \mathscr{L}(E)$, $U_{v \otimes y^{\prime}} \in \mathscr{L}^{\#}\left(F^{\prime}\right)$, and a simple computation yields $S_{x^{\prime} \otimes y^{\prime}}=U_{v \otimes y^{\prime}} S_{1} V_{x^{\prime} \otimes u_{0}} \in \mathscr{M}$, which was to be established.

Let us now return to the tensor product $E \otimes F$. Let $\alpha$ be a tensorial norm of $E \otimes F$. We have already seen that $\left(E \otimes_{\alpha} F\right)^{\prime}$ is a vector subspace of $\mathscr{L}\left(E, F^{\prime}\right)$. We shall say that $\alpha$ is modular if $\left(E \otimes_{\alpha} F\right)^{\prime}$ is an $\mathscr{L}^{\#}\left(F^{\prime}\right)$-left and an $\mathscr{L}(E)$-right submodule of $\mathscr{L}\left(E, F^{\prime}\right)$. See the previous section for the basic conventions regarding the duality of tensor products. We shall say that a modular norm $\alpha$ is strongly modular if, in addition, $\left(E \otimes_{\alpha} F\right)^{\prime}$ is an $\mathscr{L}\left(F^{\prime}\right)$-left submodule. We would like to emphasize the purely algebraic character of the definition of the concept of modular norms. In spite of this the concept has many interesting topological connections. In fact, as we shall see, it turns out to be the topological analogue of the metric concept of uniform cross norm due to Schatten [14, Theorem 4.1, p. 62]. Since uniform norms will also play a roll in the present work, we discuss them now in some detail. For more details, we refer the reader to our previous discussions of uniform cross norms in [7] and [10].

Let $\mathfrak{t} \in E \otimes F$ have representation (3.1), $V \in \mathscr{L}(E)$ and $W \in \mathscr{L}(F)$. We define $V \otimes W(\mathrm{t})$ as

$$
V \otimes W(\mathrm{t})=\sum_{i=1}^{n} V x_{i} \otimes W y_{i}
$$


Clearly $V \otimes W(t) \in E \otimes F$ and this tensor is independent of the particular representation (3.1) of $\mathrm{t}$. Thus, the symbol $V \otimes W$ defines a linear transformation of $E \otimes F$ into itself. We say that a tensorial norm $\alpha$ of $E \otimes F$ is uniform if, for every $\mathrm{t} \in E \otimes F, V \in \mathscr{L}(E)$ and $W \in \mathscr{L}(F)$ we have

$$
\alpha[V \otimes W(\mathrm{t})] \leqq\|V\|\|W\| \alpha(\mathrm{t}) .
$$

We are going to see later that every modular norm is equivalent to a uniform norm and that every modular cross norm is equivalent to a uniform cross norm, but first we must develop the basic theory of modular norms.

Let us now return to a modular norm $\alpha$ of $E \otimes F$. Since, by definition, $\left(E \otimes_{\alpha} F\right)^{\prime}$ is a submodule of $\mathscr{L}\left(E, F^{\prime}\right)$ under the appropriate module structure, we have that $\left(E \otimes_{\alpha} F\right)^{\prime}$ itself is an $\mathscr{L}^{\#}\left(F^{\prime}\right)$-left and an $\mathscr{L}(E)$-right module. The content of the next theorem is that this structure of module is topological (in the sense of $\S 2$ ), with respect to the topology defined by the dual norm $\alpha^{\prime}$ on $\left(E \otimes_{\alpha} F\right)^{\prime}$.

THEOREM 4.2. Let $\alpha$ be a modular norm of $E \otimes F$. Then $\left(E \otimes_{\alpha} F\right)^{\prime}$ is a topological $\mathscr{L}^{\#}\left(F^{\prime}\right)$-left and a topological $\mathscr{L}(E)$-right module. Equivalently, there exists a number $k_{1}>0$ such that, for every $U \in \mathscr{L}^{\#}\left(F^{\prime}\right), S \in\left(E \otimes_{\alpha} F\right)^{\prime}$ and $V \in \mathscr{L}(E)$, we have

$$
\alpha^{\prime}(U S V) \leqq k_{1}\|U\|\|V\| \alpha^{\prime}(S)
$$

Proof. It follows from the fact that $\alpha$ is majorized by $\gamma(\S 3)$ that $\alpha^{\prime}$ majorizes the transformation norm on $\left(E \otimes_{\alpha} F\right)^{\prime} \subset \mathscr{L}\left(E, F^{\prime}\right)$. This was shown by Schatten [14, Lemma 3.2, p. 45] for a cross norm $\alpha$, but the extension to tensorial norms is immediate. Our result now follows from this and the change-of-norm theorem (Theorem 2.2) above.

For strongly modular norms we get, of course, the corresponding result.

THEOREM 4.3. Let $\alpha$ be a strongly modular norm of $E \otimes F$. Then $\left(E \otimes_{\alpha} F\right)^{\prime}$ is a topological $\mathscr{L}\left(F^{\prime}\right)$-left and a topological $\mathscr{L}(E)$-right module.

Let us now consider the tensor product $E^{\prime} \otimes F^{\prime}$. If $\alpha$ is a modular norm of $E \otimes F$, then, it follows from Theorem 4.1 that $\left(E \otimes_{\alpha} F\right)^{\prime}$ contains the natural image of $E^{\prime} \otimes F^{\prime}$ in $\mathscr{L}\left(E, F^{\prime}\right)$. Consequently the dual norm $\alpha^{\prime}$ of $\left(E \otimes_{\alpha} F\right)^{\prime}$ induces a norm on $E^{\prime} \otimes F^{\prime}$, rendering isometric the natural imbedding of $E^{\prime} \otimes F^{\prime}$ into $\left(E \otimes_{\alpha} F\right)^{\prime}$. We continue to designate this new norm by $\alpha^{\prime}$, since there is no danger of ambiguity.

THEOREM 4.4. If $\alpha$ is a modular cross norm on $E \otimes F$, then the norm $\alpha^{\prime}$ on $E^{\prime} \otimes F^{\prime}$ is admissible (§3).

Proof. We have already seen (see the proof of Theorem 4.3) that $\alpha^{\prime}$ majorizes the transformation norm on $\left(E \otimes_{\alpha} F\right)^{\prime} \subset \mathscr{L}\left(E, F^{\prime}\right)$. It follows from this and the fact that the least cross norm $\lambda$ on $E^{\prime} \otimes F^{\prime}$ renders isometric the natural mapping of $E^{\prime} \otimes F^{\prime}$ into $\mathscr{L}\left(E, F^{\prime}\right)$, that $\alpha^{\prime}$ on $E^{\prime} \otimes F^{\prime}$ majorizes $\lambda$. It remains to prove that 
$\alpha^{\prime}$ on $E^{\prime} \otimes F^{\prime}$ is majorized by the greatest cross norm $\gamma$. Clearly we may assume that $E^{\prime}$ and $F^{\prime}$ contain functionals $x_{0}^{\prime}$ and $y_{0}^{\prime}$, different from zero and we might as well assume that they are unit functionals. Then $x_{0}^{\prime} \otimes y_{0}^{\prime}$ is a kernel of a transformation $S_{x_{0}^{\prime} \otimes y_{0}^{\prime}} \in\left(E \otimes_{\alpha} F\right)^{\prime}$. Let now $x^{\prime}$ and $y^{\prime}$ be arbitrary elements of $E^{\prime}$ and $F^{\prime}$, respectively and $x_{0} \in E$ and $y_{0} \in F$, such that $\left\langle x_{0}, x_{0}^{\prime}\right\rangle=\left\langle y_{0}, y_{0}^{\prime}\right\rangle=1$. Then $U_{y_{0} \otimes y^{\prime}} \in \mathscr{L}^{\prime}\left(F^{\prime}\right), V_{x^{\prime} \otimes x_{0}} \in \mathscr{L}(E)$, and

$$
S_{x^{\prime} \otimes y^{\prime}}=U_{y_{0} \otimes y^{\prime}} S_{x_{0}^{\prime} \otimes y_{0}^{0}} V_{x^{\prime} \otimes x_{0}} .
$$

From here and Theorem 4.2 we get

$$
\begin{aligned}
\alpha^{\prime}\left(x^{\prime} \otimes y^{\prime}\right)=\alpha^{\prime}\left(S_{x^{\prime} \otimes y^{\prime}}\right) & \leqq k_{1}\left\|U_{y_{0} \otimes y^{\prime}}\right\|\left\|V_{x^{\prime} \otimes x_{0}}\right\| \alpha^{\prime}\left(S_{x_{0}^{\prime} \otimes y_{0}^{\prime}}\right) \\
& \leqq k_{1}\left\|y_{0}\right\|\left\|y^{\prime}\right\|\left\|x^{\prime}\right\|\left\|x_{0}\right\| \alpha^{\prime}\left(S_{x_{0}^{\prime} \otimes y_{0}}\right) .
\end{aligned}
$$

Since $y_{0}$ and $x_{0}$ may be chosen in such a way that $\left\|x_{0}\right\|$ and $\left\|y_{0}\right\|$ are arbitrarily close to 1 , we finally get

$$
\alpha^{\prime}\left(x^{\prime} \otimes y^{\prime}\right) \leqq k_{1}\left\|x^{\prime}\right\|\left\|y^{\prime}\right\| \alpha^{\prime}\left(S_{x_{0}^{\prime} \otimes y_{0}^{\prime}}\right) .
$$

Let now $s$ be any tensor of $E^{\prime} \otimes F^{\prime}$ with representation

$$
\mathfrak{s}=\sum_{j=1}^{m} x_{j}^{\prime} \otimes y_{j}^{\prime},
$$

we have

$$
\alpha^{\prime}(\mathfrak{g}) \leqq k_{1} \alpha^{\prime}\left(S_{x_{0}^{\prime} \otimes y_{0}^{\prime}}\right) \sum_{j=1}^{m}\left\|x_{j}^{\prime}\right\|\left\|y_{j}^{\prime}\right\| .
$$

Since (4.8) is valid for any representation (4.7), it follows from the definition of $\gamma$ (see (3.4)) that $\alpha^{\prime}(\mathfrak{g}) \leqq k_{1} \alpha^{\prime}\left(S_{x_{0}^{\prime} \otimes y_{0}^{\prime}}\right) \gamma(\mathfrak{g})$. This completes our proof.

THEOREM 4.5. Every modular norm of $E \otimes F$ is admissible.

Proof. Schatten has shown [14, Theorem 2.1, p. 32] that a cross norm $\alpha$ of $E \otimes F$ is ordinary if and only if $\alpha^{\prime}$ on $E^{\prime} \otimes F^{\prime}$ is ordinary. This is in essence a topological (as opposed to metric) fact and extends to the present situation, yielding the corresponding result that a tensorial norm $\alpha$ is admissible if and only if $\alpha^{\prime}$ on $E^{\prime} \otimes F^{\prime}$ is admissible. Now in Theorem 4.4 we proved that if $\alpha$ is a modular norm of $E \otimes F, \alpha^{\prime}$ is admissible on $E^{\prime} \otimes F^{\prime}$. It follows then that $\alpha$, itself, must be admissible. This completes our proof.

Our next theorem concerns the relation between modular norms and the transformations $V \otimes W, V \in \mathscr{L}(E), W \in \mathscr{L}(F)$, defined in the beginning of the present section.

THEOREM 4.6. Let $\alpha$ be a modular norm of $E \otimes F, V \in \mathscr{L}(E)$, and $W \in \mathscr{L}(F)$. Then the linear transformation $V \otimes W$, defined by (4.1), of $E \otimes F$ into itself can be extended (uniquely, of course) to a bounded linear operator on $E \otimes_{\alpha} F$. 
Proof. It suffices to show that the linear transformation $V \otimes W$ of $E \otimes F$ into itself is bounded with respect to $\alpha$. So let $\mathrm{t} \in E \otimes F$ such that $\alpha(\mathrm{t}) \leqq 1$ and $S \in\left(E \otimes_{\alpha} F\right)^{\prime}$, such that $\alpha^{\prime}(S) \leqq 1$. By a simple computation one verifies that

$$
\langle V \otimes W(\mathrm{t}), S\rangle=\left\langle\mathrm{t}, W^{\prime} S V\right\rangle .
$$

Now, by Theorem 4.2, one has $\alpha^{\prime}\left(W^{\prime} S V\right) \leqq k_{1}\left\|W^{\prime}\right\|\|V\| \leqq k_{1}\|W\|\|V\|$. From this and (4.9) one gets that $\alpha[V \otimes W(\mathrm{t})] \leqq k_{1}\|V\|\|W\|$ for every $\mathfrak{t} \in E \otimes F$, such that $\alpha(\mathrm{t}) \leqq 1$. From this our conclusion follows.

We use the symbol $V \otimes W$ to denote also the operator resulting from the extension described in Theorem 4.6. We call this operator the Kronecker product $\left(^{6}\right)$ of $V$ and $W$, because in finite dimensional spaces the matrix of $V \otimes W$ is the Kronecker product, in the classical sense, of the matrices of $V$ and $W$. Actually for any modular norm $\alpha$ of $E \otimes F$, the tensor product $\mathscr{L}(E) \otimes \mathscr{L}(F)$ can be mapped linearly into $\mathscr{L}\left(E \otimes_{\alpha} F\right)$, via the Kronecker product. This mapping is isomorphic, but this aspect will not be pursued here because it is discussed in great detail in [7] for uniform cross norms and the extension to the present situation is, once we have Theorem 4.6, immediate. It is not hard to see from the proof of this theorem that, for every $V \in \mathscr{L}(E), W \in \mathscr{L}(F)$ and $\mathrm{t} \in E \otimes_{\alpha} F$,

$$
\alpha[V \otimes W(\mathrm{t})] \leqq k_{1}\|V\|\|W\| \alpha(\mathrm{t})
$$

where $k_{1}$ is the constant appearing in that proof. Theorem 4.6 itself is a generalization of [10, Theorem 1.1]. However, in the present more general situation, we have a converse. We shall now prepare the ground for it.

The symbol $I$ will stand for the identity operator on any space which we may happen to be discussing. The context will take care of possible ambiguities. Let us now assume that $\alpha$ is a tensorial norm of $E \otimes F$ with the property that every linear transformation of $E \otimes F$ into itself defined by (4.1) can be extended to a bounded operator (the Kronecker product) $V \otimes W$ on $E \otimes_{\alpha} F$. We shall describe this situation by saying that the Kronecker product is defined as an operator on $E \otimes_{\alpha} F$. For such an $\alpha$ let us define a structure of left module on $E \otimes_{\alpha} F$ over $\mathscr{L}(E)$ and a structure of right module over $\mathscr{L}(F)$, as follows. For $V \in \mathscr{L}(E), W \in \mathscr{L}(F)$, and $\mathrm{t} \in E \otimes_{\alpha} F$, we define

$$
V \cdot \mathrm{t}=V \otimes I(\mathrm{t})
$$

and

$$
\mathfrak{t} \cdot W=I \otimes W(\mathfrak{t}) .
$$

LEMMA 4.1. Let $\alpha$ be a tensorial norm of $E \otimes F$, for which the Kronecker product (4.1) can be extended to an element of $\mathscr{L}\left(E \otimes_{\alpha} F\right)$, for every $V \in \mathscr{L}(E)$ and every $W \in \mathscr{L}(F)$. Then $E \otimes_{\alpha} F$ is a topological left module over $\mathscr{L}(E)$ and a topological right module over $\mathscr{L}(F)$ under the structure defined by (4.11) and (4.12).

${ }^{6}$ ) With respect to $\alpha$, should there be any possible ambiguity. 
Proof. We only prove that the expression $V \cdot t$ is jointly continuous with respect to the various given topologies. As we have seen several times, the rest consists of several repetitions of the same argument. Also as remarked earlier, it suffices to establish the separate continuity of $V \cdot t$. Continuity with respect to $t$, for fixed $V$ follows readily from the hypothesis. So let us fix $\mathrm{t} \in E \otimes_{\alpha} F$ and show that the linear transformation $V \rightarrow V \cdot t$ of $\mathscr{L}(E)$ into $E \otimes_{\alpha} F$ is bounded. This follows from the uniform boundedness theorem as follows. Let us first assume that $t$ is a finite tensor, i.e., an element of the algebraic tensor product $E \otimes F$. Now, we know that the greatest cross norm $\gamma$ is uniform [14, Lemma 2.13, p. 37] and we are assuming that $\alpha$ is tensorial. These two facts yield the result that there is a number $\kappa>0$ such that

$$
\alpha(V \cdot \mathrm{t}) \leqq \kappa \gamma(V \cdot \mathrm{t})=\kappa \gamma[V \otimes I(\mathrm{t})] \leqq \kappa\|V\| \gamma(\mathrm{t}) .
$$

It follows then, that for a finite tensor $\mathrm{t}, V \rightarrow V \cdot \mathrm{t}$ is a bounded linear transformation of $\mathscr{L}(E)$ into $E \otimes_{\alpha} F$. Let $\mathrm{t}$ be now an arbitrary element of $E \otimes_{\alpha} F$. Then there exists a sequence $\mathrm{t}_{1}, \mathrm{t}_{2}, \cdots$ of finite tensors converging to $\mathrm{t}$ in $E \otimes_{\alpha} F$. For each $V$, the bounded linear transformations $V \rightarrow V \cdot \mathrm{t}_{n}$ converge to $V \rightarrow V \cdot \mathrm{t}$ in $E \otimes_{\alpha} F$. By the uniform boundedness theorem, the latter transformation must be bounded. This completes our proof.

It might be of interest to remark at this point that in terms of bounded linear transformations, the operations $V \cdot t$ and $t \cdot W$, defined for tensors, correspond to composition of transformations in the sense that if $\alpha$ is admissible, then $V \cdot t$ is a kernel of the transformation $T_{\mathrm{t}} V^{\prime}$, while $\mathrm{t} \cdot W$ is a kernel of $W T_{\mathrm{t}}$.

THEOREM 4.7. Let $\alpha$ be a tensorial norm of $E \otimes F$. A necessary and sufficient condition in order that the Kronecker product of any two $V \in \mathscr{L}(E)$ and $W \in \mathscr{L}(F)$ be well defined as an operator on $E \otimes_{\alpha} F$ is that $\alpha$ be modular.

Proof. Sufficiency is a restatement of Theorem 4.6. Let us prove necessity. Suppose that every such Kronecker product is well defined, and let $U \in \mathscr{L}^{\#}\left(F^{\prime}\right)$, $S \in\left(E \otimes_{\alpha} F\right)^{\prime}$ and $V \in \mathscr{L}(E)$. We must show that $U S V \in\left(E \otimes_{\alpha} F\right)^{\prime}$. It suffices to show that for finite tensors $\mathrm{t} \in E \otimes F$, the expression $\langle\mathrm{t}, U S V\rangle$, which is well defined, behaves like a functional on $E \otimes F$ which is bounded with respect to $\alpha$. But a simple computation yields

$$
\langle\mathrm{t}, U S V\rangle=\left\langle V \cdot \mathrm{t} \cdot U^{\#}, S\right\rangle,
$$

and the last expression in (4.13) behaves, by Lemma 4.1, as such a bounded functional. This completes the proof of necessity.

One can now see from Lemma 4.1 and Theorem 4.7 that the concept of modular norm, defined in purely algebraic terms, is the topological analogue of the metric concept of uniform norm, and that a uniform norm is modular.

Let us return to the tensor product $E^{\prime} \otimes F^{\prime}$. We know that if $\alpha$ is a modular norm of $E \otimes F$, then $\alpha^{\prime}$ is a well defined admissible norm of $E^{\prime} \otimes F^{\prime}$. One might 
ask if $\alpha^{\prime}$ is modular. We do not know the answer to this question, but we have the following related theorem.

THEOREM 4.8. Suppose that $E$ is reflexive and that $\alpha$ is a strongly modular norm of $E \otimes F$. Then $\alpha^{\prime}$ is a modular norm of $E^{\prime} \otimes F^{\prime}$.

Proof. Suppose that $\alpha$ is strongly modular. By Theorem 4.7, it suffices to show that, for every $U \in \mathscr{L}\left(F^{\prime}\right)$ and $T \in \mathscr{L}\left(E^{\prime}\right)$, the Kronecker product $T \otimes U$ on $E^{\prime} \otimes F^{\prime}$ is a bounded linear transformation with respect to $\alpha^{\prime}$. We use the natural isometry of $E^{\prime} \otimes_{\alpha^{\prime}} F^{\prime}$ into $\left(E \otimes_{\alpha} F\right)^{\prime}$. Since $E$ is reflexive, $T$ is the transpose of some bounded operator $V \in \mathscr{L}(E)$. Now, for $\mathfrak{s} \in E^{\prime} \otimes F^{\prime}$, a simple computation yields that $T \otimes U(\mathfrak{s})$ is a kernel of $U S_{3} V \in\left(E \otimes_{\alpha} F\right)^{\prime}$ and the isometry just mentioned, together with Theorem 4.2, yields the result.

It was first discovered by Schatten [14] that the norms $\lambda$ and $\gamma$ are uniform, hence modular. It follows easily from (3.16) above that $\gamma$ is even strongly modular. The corresponding result for $\lambda$ is considerably more difficult, but it was established (using different terminology) by Grothendieck [11, Theorem 10, p. 132], and we omit all details here.

5. The cross norm $\pi$. Before proceeding with the definition of $\pi$ it might be well to say a few words to motivate the introduction of this norm, which seems to be new, in so far as the author knows. The main results to be presented in the next sections were originally obtained with the help of the greatest cross norm $\gamma$ on $F^{\prime} \otimes F$. Later it was realized that the arguments, as originally formulated were valid only for reflexive spaces or perhaps for other somewhat more general, but still quite restrictive, classes of spaces. It fortunately turns out that the main theorems themselves are valid in general, but that in the arguments $\gamma$ had to be replaced by the present norm $\pi$, which is only defined for tensor products $\left({ }^{7}\right)$ of the form $F^{\prime} \otimes F$, and can often be made to play the role of $\gamma$ for nonreflexive spaces. By (3.16) we know that $\mathscr{L}\left(F^{\prime}\right)$ is the dual space of $F^{\prime} \otimes_{\gamma} F$. Hence, for $\mathfrak{w} \in F^{\prime} \otimes F$,

$$
\gamma(\mathfrak{w})=\sup |\operatorname{tr}(U \times \mathfrak{w})|,
$$

where the sup is taken for every $U \in \mathscr{L}\left(F^{\prime}\right)$ such that $\|U\| \leqq 1$. Now we have already seen that, in connection with modular norms, it is $\mathscr{L}^{\#}\left(F^{\prime}\right)$, rather than $\mathscr{L}\left(F^{\prime}\right)$, which interests us. Thus we define in a manner analogous to (5.1), for $\mathfrak{w} \in F^{\prime} \otimes F$,

$$
\pi(\mathfrak{w})=\sup |\operatorname{tr}(U \times \mathfrak{w})|,
$$

where now the sup is taken for all $U \in \mathscr{L}^{\#}\left(F^{\prime}\right)$, such that $\|U\| \leqq 1$. We will show shortly that $\pi$ is indeed a cross norm and will proceed to investigate its more immediate properties. Its introduction merely reflects the ignorance of the present author, who cannot determine whether or not $\pi$ is indeed different from $\gamma$. A

(7) And, by symmetry, for $F \otimes F^{\prime}$. 
similar doubt lead Grothendieck [11] to introduce his nuclear norm, which is not yet actually known to be different from $\gamma$. We have not investigated the relation between our norm $\pi$ and the nuclear norm.

We show that $\pi$ is indeed a norm. The triangle inequality and the homogeneity follow from usual arguments, which we do not repeat. All that remains to be shown is that $\pi(\mathfrak{w})=0$ implies $\mathfrak{w}=0$. Let $\mathfrak{w}$ have a representation

$$
\mathfrak{w}=\sum_{i=1}^{n} y_{i}^{\prime} \otimes y_{i} .
$$

Now, if $\pi(\mathfrak{w})=0$, then $\operatorname{tr} U \times \mathfrak{w}=0$, for every $U \in \mathscr{L}^{\#}\left(F^{\prime}\right)$. Also, by Lemma 1.1, p. 20 of [14], we can assume that in (5.3) the sets $y_{1}^{\prime}, y_{2}^{\prime}, \cdots, y_{n}^{\prime}$ and $y_{1}, y_{2}, \cdots, y_{n}$ are linearly independent. For each $i$ we find $v_{i}^{\prime} \in F^{\prime}$, with the property that $\left\langle y_{i}, v_{i}^{\prime}\right\rangle=\left\|y_{i}\right\|$. Now, there is a bounded linear transformation $Y$ from the space $F_{1}^{\prime}$ generated by the set $y_{1}^{\prime}, y_{2}^{\prime}, \cdots, y_{n}^{\prime}$ into the space generated by the set $v_{1}^{\prime}, v_{2}^{\prime}, \cdots$, $v_{n}^{\prime}$, such that $Y y_{i}^{\prime}=v_{i}^{\prime}$, for every $i$. On the other hand, since $F_{1}^{\prime}$ is finite dimensional, there exists a projection $Z$ of $F^{\prime}$ onto $F_{1}^{\prime}$ which is a continuous operator of $F^{\prime}$, when the latter is given the weak* topology, i.e., $Z \in \mathscr{L}^{*}\left(F^{\prime}\right)$. It is not hard to see that $U=Y Z \in \mathscr{L}^{\#}\left(F^{\prime}\right)$ and that

$$
\operatorname{tr} U \times \mathfrak{w}=\sum_{i=1}^{n}\left\|y_{i}\right\|=0 .
$$

Hence $\mathfrak{w}=0$. This completes our demonstration that $\pi$ is a norm.

THEOREM 5.1. The norm $\pi$ on $F^{\prime} \otimes F$ is an ordinary norm.

Proof. Comparing (5.1) and (5.2) we get immediately that $\pi \leqq \gamma$. We show $\pi \geqq \lambda$. From the definition of $\lambda$ in $\S 3$, we get, for $\mathfrak{w} \in F^{\prime} \otimes F$, represented by (5.3),

$$
\lambda(\mathfrak{w})=\sup \left|\sum_{i=1}^{n}\left\langle v, y_{i}^{\prime}\right\rangle\left\langle y_{i}, v^{\prime}\right\rangle\right|,
$$

where the sup is taken for all $v \in F$ and $v^{\prime} \in F^{\prime}$ of norm less than or equal to one. Now $v \otimes v^{\prime} \in F \otimes F^{\prime}$ is a kernel of $T_{v \otimes v^{\prime}}: F^{\prime} \rightarrow F^{\prime}$, an operator of norm $\leqq 1$. One sees without difficulty that (5.4) can be written as

$$
\lambda(\mathfrak{w})=\sup \left|\operatorname{tr} T_{v \otimes v^{\prime}} \times \mathfrak{w}\right|,
$$

and comparison with (5.2) gives the desired inequality, since $T_{v \otimes v^{\prime}}$ has norm $\leqq 1$, and is contained in $\mathscr{L}^{\#}\left(F^{\prime}\right)$. Hence $\pi$ is an ordinary norm.

The norm $\pi$ is then an admissible cross norm. We will be primarily interested in the complete tensor product $F^{\prime} \otimes_{\pi} F$.

If we interchange the order of $F^{\prime}$ and $F$ in our considerations we are led to study $F \otimes F^{\prime}$. It will be useful for the work in later sections to introduce a norm $\pi$ in this space also. One might do it analogously by looking for the appropriate subspace of $\mathscr{L}\left(F, F^{\prime \prime}\right)$ with respect to which one might write an expression similar to that in 
(5.2). However, for the sake of simplicity, as well as for the convenience in later discussions it is best to define $\pi$ as the norm that renders isometric the transposition of tensors defined in $\S 3$. Let $\mathfrak{u} \in F \otimes F^{\prime}$ be given by

$$
\mathfrak{u}=\sum_{i=1}^{n} y_{i} \otimes y_{i}^{\prime}
$$

$y_{i} \in F$ and $y_{i}^{\prime} \in F^{\prime}$. Then the transpose tensor $\mathfrak{u}^{\prime}$ belongs to $F^{\prime} \otimes F$ and is given, (see $\S 3)$ by

$$
\mathfrak{u}^{\prime}=\sum_{i=1}^{n} y_{i}^{\prime} \otimes y_{i}
$$

We then define

$$
\pi(\mathfrak{u})=\pi\left(\mathfrak{u}^{\prime}\right) .
$$

Clearly $\pi$ is a cross norm on $F \otimes F^{\prime}$ and we can consider the completion $F \otimes_{\pi} F^{\prime}$.

Quite likely, $\pi$ is not a modular cross norm, but the next theorem gives a property quite related to uniformity (hence modularity) which will be useful soon.

THEOREM 5.2. Let $U \in \mathscr{L}^{\#}\left(F^{\prime}\right)$ and $W \in \mathscr{L}(F)$. Then the Kronecker product $U \otimes W$ is well defined as a bounded linear operator of $F^{\prime} \otimes_{\pi} F$. What is more, for $\mathfrak{w} \in F^{\prime} \otimes_{\pi} F$,

$$
\pi[U \otimes W(\mathfrak{w})] \leqq\|U\|\|W\| \pi(\mathfrak{w})
$$

Proof. It suffices to establish the inequality (5.9) for finite tensors $\mathfrak{w} \in F^{\prime} \otimes F$. The rest of the theorem follows easily from this. So let $\mathfrak{w} \in F^{\prime} \otimes F$ have a representation (5.3), then

$$
U \otimes W(\mathfrak{w})=\sum_{i=1}^{n} U y_{i}^{\prime} \otimes W y_{i}
$$

Suppose $U_{1} \in \mathscr{L}^{\#}\left(F^{\prime}\right)$ and let $\left\|U_{1}\right\| \leqq 1$. The following relation is easy to establish.

$$
\begin{aligned}
\left\langle U \otimes W(\mathfrak{w}), U_{1}\right\rangle & =\operatorname{tr}\left\{U_{1} \times[U \otimes W(\mathfrak{w})]\right\}=\operatorname{tr}\left\{\left(W^{\prime} U_{1} U\right) \times \mathfrak{w}\right\} \\
& =\left\langle\mathfrak{w}, W^{\prime} U_{1} U\right\rangle .
\end{aligned}
$$

Now we have, since $W^{\prime}, U_{1}$, and $U$ are elements of $\mathscr{L}^{\#}\left(F^{\prime}\right)$, that $W^{\prime} U_{1} U$ belongs to $\mathscr{L}^{\#}\left(F^{\prime}\right)$, and it follows from (5.11) and the definition of $\pi$, that

$$
\begin{aligned}
\pi[U \otimes W(\mathfrak{w})] & =\sup \left|\operatorname{tr}\left\{U_{1} \times[U \otimes W(\mathfrak{w})]\right\}\right| \\
& \leqq\|U\|\|W\| \pi(\mathfrak{w}) .
\end{aligned}
$$

In (5.12) the sup is taken for all $U_{1}$ in $\mathscr{L}^{\#}\left(F^{\prime}\right)$ with $\left\|U_{1}\right\| \leqq 1$. This completes the proof.

It follows from Theorem 5.2, in a manner completely analogous to our earlier discussion on modular norms that $F^{\prime} \otimes_{\pi} F$ is a left topological module over $\mathscr{L}^{\#}\left(F^{\prime}\right)$ 
and a right topological module over $\mathscr{L}(F)$, the structure defined by the trilinear mapping $U \cdot \mathfrak{w} \cdot W$

$$
U \cdot \mathfrak{w} \cdot W=U \otimes W(\mathfrak{w}),
$$

for $U \in \mathscr{L}^{\#}\left(F^{\prime}\right), \mathfrak{w} \in F^{\prime} \otimes_{\pi} F$, and $W \in \mathscr{L}(F)$. A theorem similar to Theorem 5.2 holds for $F \otimes_{\pi} F^{\prime}$. Hence we can define $F \otimes_{\pi} F^{\prime}$. as a left module over $\mathscr{L}(F)$ and a right module over $\mathscr{L}^{\#}\left(F^{\prime}\right)$ under a trilinear form $W \cdot \mathfrak{u} \cdot U$, defined by

$$
W \cdot \mathfrak{u} \cdot U=\left[U \cdot\left(\mathfrak{u}^{\prime}\right) \cdot W\right]^{\prime} .
$$

We have seen at the end of $\$ 3$ that the trace

$$
\operatorname{tr} \mathfrak{w}=\sum_{i=1}^{n}\left\langle y_{i}, y_{i}^{\prime}\right\rangle
$$

defined for finite tensors $\mathfrak{w}$ by (5.3), can be extended to tensors in $F^{\prime} \otimes_{\gamma} F$. We can also see here that such an extension is possible even for tensors in $F^{\prime} \otimes_{\pi} F$, since it follows easily from the definition of $\pi$ that

$$
|\operatorname{tr} \mathfrak{w}| \leqq \pi(\mathfrak{w}) \text {. }
$$

Thus the trace behaves like a bounded linear functional with respect to $\pi$ and can be extended to $F^{\prime} \otimes_{\pi} F$ and the extended functional still satisfies (5.16). The trace for an element $\mathfrak{u} \in F \otimes_{\pi} F^{\prime}$ is defined by

$$
\operatorname{tr} \mathfrak{u}=\operatorname{tr}\left[(\mathfrak{u})^{\prime}\right],
$$

and (5.16) is again satisfied.

We turn now to the discussion of the duality of the spaces $F^{\prime} \otimes_{\pi} F$ and $F \otimes_{\pi} F^{\prime}$, giving details only for $F^{\prime} \otimes_{\pi} F$. We have the following preliminary result.

TheOREM 5.3. Let $U \in \mathscr{L}^{\#}\left(F^{\prime}\right)$. Then, for every $\mathfrak{w} \in F^{\prime} \otimes F, U \times \mathfrak{w}$ and $\mathfrak{w} \times U$ both belong to $F^{\prime} \otimes F$, and the mappings $\mathfrak{w} \rightarrow U \times \mathfrak{w}$ and $\mathfrak{w} \rightarrow \mathfrak{w} \times U$ can both be extended to bounded linear operators, designated again by $\mathfrak{w} \rightarrow U \times \mathfrak{w}$ and $\mathfrak{w} \rightarrow \mathfrak{w} \times U$, of $F^{\prime} \otimes_{\pi} F$. Further, for $\mathfrak{w} \in F^{\prime} \otimes_{\pi} F$ and $U \in \mathscr{L}^{\#}\left(F^{\prime}\right)$, we have

$$
\langle\mathfrak{w}, U\rangle=\operatorname{tr} U \times \mathfrak{w}=\operatorname{tr} \mathfrak{w} \times U .
$$

Proof. It is obvious from the definition $(\$ 3,(3.13))$ of $\mathfrak{w} \times U$ that it belongs to $F^{\prime} \otimes F$. As to $U \times \mathfrak{w}$, this follows from the fact that for every $y \in F, U^{\prime} y$ is again in $F$. To establish the extensibility, thereby completing the proof of the theorem, it suffices to show that the original mappings of $F^{\prime} \otimes F$ into itself are bounded with respect to $\pi$. We do it for $\mathfrak{w} \times U$, which is somewhat more tractable. The proof for $U \times \mathfrak{w}$ follows similar lines. Now for $\mathfrak{w} \in F^{\prime} \otimes F$,

$$
\begin{aligned}
\pi(\mathfrak{w} \times U) & =\sup \left|\operatorname{tr}\left[(\mathfrak{w} \times U) \times U_{1}\right]\right|=\sup \left|\operatorname{tr}\left(\mathfrak{w} \times U_{1} U\right)\right| \\
& \leqq \sup \left[\left\|U_{1} U\right\| \pi(\mathfrak{w})\right] \leqq\|U\| \pi(\mathfrak{w}),
\end{aligned}
$$

where the sup is taken as $U_{1}$ ranges over the set of all $U_{1} \in \mathscr{L}^{\#}\left(F^{\prime}\right)$ with operator norm $\leqq 1$. As to (5.18) it is obviously valid for finite tensors and extensible to arbitrary tensors by the usual continuity arguments. 
As to the space $F \otimes_{\pi} F^{\prime}$, since $\pi$ is defined in terms of the transposition transformation, we might as well identify the dual of $F \otimes_{\pi} F^{\prime}$ with that of $F^{\prime} \otimes_{\pi} F$, a subspace of $\mathscr{L}\left(F^{\prime}\right)$, instead of, as the conventions in $\S 3$ dictate, with a subspace of $\mathscr{L}\left(F, F^{\prime \prime}\right)$. Then the rephrasing of Theorem 5.3, which applies to $F \otimes_{\pi} F^{\prime}$, is immediate, and in the sequel we shall use the result obtained without further specific reference. The next theorem is perhaps of greater interest, since in a way it characterizes the elements of $\left(F^{\prime} \otimes_{\pi} F\right)^{\prime}$ among those of $\mathscr{L}\left(F^{\prime}\right)$.

TheOREM 5.4. Let $U \in \mathscr{L}\left(F^{\prime}\right)$. Then $U \in\left(F^{\prime} \otimes_{\pi} F\right)^{\prime}=\left(F \otimes_{\pi} F^{\prime}\right)^{\prime}$, if and only if the mapping $\mathfrak{w} \rightarrow \mathfrak{w} \times U\left(\mathfrak{u} \rightarrow \mathfrak{u} \times U^{\#}\right)$ of $F^{\prime} \otimes F\left(F \otimes F^{\prime}\right)$ into itself can be extended to a bounded linear operator of $F^{\prime} \otimes_{\pi} F\left(F \otimes_{\pi} F^{\prime}\right)$ designated again by $\mathfrak{w} \rightarrow \mathfrak{w} \times U$ $\left(\mathfrak{u} \rightarrow \mathfrak{u} \times U^{\#}\right)$. For $\mathfrak{w} \in F^{\prime} \otimes_{\pi} F\left(\mathfrak{u} \in F \otimes_{\pi} F^{\prime}\right)$ and $U \in\left(F^{\prime} \otimes_{\pi} F\right)^{\prime}=\left(F \otimes_{\pi} F^{\prime}\right)^{\prime}$ we have

$$
\langle\mathfrak{w}, U\rangle=\operatorname{tr} \mathfrak{w} \times U,
$$

and

$$
\langle\mathfrak{u}, U\rangle=\operatorname{tr} \mathfrak{u} \times U^{\#} .
$$

Further, for $U \in\left(F^{\prime} \otimes_{\pi} F\right)^{\prime}=\left(F \otimes_{\pi} F^{\prime}\right)^{\prime}, \mathfrak{w} \in F^{\prime} \otimes_{\pi} F$ and $\mathfrak{u} \in F \otimes_{\pi} F^{\prime}$, we have

$$
\pi(\mathfrak{w} \times U) \leqq \pi^{\prime}(U) \pi(\mathfrak{w})
$$

and

$$
\pi\left(\mathfrak{u} \times U^{\#}\right) \leqq \pi^{\prime}(U) \pi(\mathfrak{u}) .
$$

Proof (for $\left.F^{\prime} \otimes_{\pi} F\right)$. Let $U \in\left(F^{\prime} \otimes_{\pi} F\right)^{\prime}$. To establish the required extensibility, it suffices to show that the mapping $\mathfrak{w} \rightarrow \mathfrak{w} \times U$ of $F^{\prime} \otimes F$ into itself is bounded with respect to $\pi$. We do this.

$$
\begin{aligned}
\pi(\mathfrak{w} \times U) & =\sup \left|\operatorname{tr}\left[U_{1} \times(\mathfrak{w} \times U)\right]\right|=\sup \left|\operatorname{tr}\left[\left(U_{1} \times \mathfrak{w}\right) \times U\right]\right| \\
& =\sup \left|\left\langle\left(U_{1} \times \mathfrak{w}\right), U\right\rangle\right| \leqq \sup \left[\pi\left(U_{1} \times \mathfrak{w}\right) \pi^{\prime}(U)\right] \\
& \leqq \pi(\mathfrak{w}) \pi^{\prime}(U) .
\end{aligned}
$$

In (5.24) the sup is taken as $U_{1}$ ranges over all the operators in $\mathscr{L}^{\#}\left(F^{\prime}\right)$ with operator norm $\leqq 1$. The last inequality in (5.24) follows from an inequality for $U \times \mathfrak{w}$, which we have omitted, analogous to (5.19). This completes the proof of the desired extensibility as well as of (5.22). The relation (5.20) (similarly, of course, for the corresponding relation (5.21)) is established in the usual manner: for finite tensors first and extended by continuity.

It remains to show that the property of extensibility characterizes the elements of $\left(F^{\prime} \otimes_{\pi} F\right)^{\prime}$. Thus, suppose that an element $U \in \mathscr{L}\left(F^{\prime}\right)$ has the desired extensibility property. This means that there exists a number $\kappa>0$ such that, for every $\mathfrak{w} \in F^{\prime} \otimes F, \pi(\mathfrak{w} \times U) \leqq \kappa \pi(\mathfrak{w})$. But then

$$
|\langle\mathfrak{w}, U\rangle|=|\operatorname{tr}(\mathfrak{w} \times U)| \leqq \kappa \pi(\mathfrak{w}) \text {. }
$$

This implies $U \in\left(F^{\prime} \otimes_{\pi} F\right)^{\prime}$. This completes the proof of our theorem. 
We have stated at the beginning of the present section that the norm $\pi$ has been introduced as a substitute, in many situations, for the norm $\gamma$. We shall examine those situations more carefully in the following section. Here we remark that the principal properties of $\pi$ which permit such a substitution are embodied in Theorems 5.3 and 5.4. It might help clarify things to try formulating the corresponding properties for $\gamma$, and to ask ourselves if $\gamma$ does actually possess them. The situation will be discussed in more detail in the next section. Here we are primarily interested in trying to illustrate. Let us take, for example, Theorem 5.4. If we consider $F^{\prime} \otimes_{\gamma} F$, its dual is now $\mathscr{L}\left(F^{\prime}\right)$. The corresponding statement in this setting would yield roughly that for every tensor $\mathfrak{w} \in F^{\prime} \otimes_{\gamma} F$ and every bounded operator $U$ on $F^{\prime}$, the tensor $\mathfrak{w} \times U$ is again a well-defined tensor of $F^{\prime} \otimes_{\gamma} F$. In the chronological development of the present work, this fact lead the author to Theorem 5.4, but in the present exposition, its proof must await further development of the theory.

We devote the remainder of this section to a discussion related to the question, posed earlier, of whether or not $\pi=\gamma$. We know very little about this question as such. An obvious sufficient condition is that $\mathscr{L}^{\#}\left(F^{\prime}\right)=\mathscr{L}\left(F^{\prime}\right)$. But this last relation holds if and only if $F$ is reflexive. Beyond this we know nothing, except for the following result.

THEOREM 5.4. A necessary and sufficient condition in order that $\pi$ be equivalent to (i.e., define the same topology as) $\gamma$ is that $\pi$ be modular.

Proof. Suppose that $\pi$ is equivalent to $\gamma$. Then $\pi$ is modular, because modularity is an algebraic property of the dual space, which is $\mathscr{L}\left(F^{\prime}\right)$ in this case, the dual of $F^{\prime} \otimes_{\gamma} F$. But $\gamma$ is modular, indeed uniform. It follows then that $\pi$ is modular.

Suppose conversely, that $\pi$ is modular. Now it follows easily from the definition of $\pi$, that $\mathscr{L}^{\#}\left(F^{\prime}\right)$ is contained in the dual space $\left(F^{\prime} \otimes_{\pi} F\right)^{\prime}$ of $F^{\prime} \otimes_{\pi} F$, in which it is actually weakly* dense. Since the identity operator of $F$ belongs to $\mathscr{L}^{\#}\left(F^{\prime}\right)$, it belongs to $\left(F^{\prime} \otimes_{\pi} F\right)^{\prime}$. It follows then from the definition of modularity that the entire $\mathscr{L}\left(F^{\prime}\right)$ is contained in $\left(F^{\prime} \otimes_{\pi} F\right)^{\prime}$, hence coincides with it. It follows then that the normed space $F^{\prime} \otimes F$ with the norm $\pi$ has the same dual space as the normed space $F^{\prime} \otimes F$ with the norm $\gamma$. We obtain by standard normed-space arguments that the two norms are equivalent. This completes our proof.

6. Duality for modular norms. Throughout this section we shall assume, with the exception of Theorems 6.3 and 6.8, without any further explicit mention, that $\alpha$ is a modular norm of $E \otimes F$, where $E$ and $F$ are, as usual, general Banach spaces. Schatten has shown [14, Definition 5.3, p. 77 and Theorem 5.12, p. 119] that the product of two Schmidt class operators is a trace class operator $\left({ }^{8}\right)$. Let $H$ be a Hilbert space. Then the Schmidt class can be identified with the tensor product $H \otimes_{\sigma} H$ (for notation see [14]), and the trace class with $H \otimes_{\gamma} H$. In this special

(8) And, conversely, that every trace class operator is the product of two Schmidt class operators. However, the converse does not seem to have a readily available generalization. 
situation $H \otimes_{\sigma} H$ is its own dual, but leaving this fact out of the picture, Schatten's theorem states that an element of $H \otimes_{\sigma} H$ times an element of its dual space is an element of $H \otimes_{\gamma} H$. The main theorem of this section contains a generalization of this theorem to perfectly general Banach spaces, where the role of $\sigma$ is played by a modular norm. To obtain such a generalization several difficulties have to be overcome. One is that, although the elements of $\left(E \otimes_{\alpha} F\right)^{\prime}$ are linear transformations, those of $E \otimes_{\alpha} F$ may not be. To circumvent this difficulty, the $\times$-products $S \times \mathrm{t}$ and $\mathrm{t} \times S$, introduced in $\S 3$ above will take the place of composition of operators. Also, unfortunately, for general Banach spaces, the norm $\gamma$ will have to be replaced by the norm $\pi$ introduced above. In the case when $\gamma=\pi$, such as for reflexive spaces, we obtain a more striking result.

We now proceed to discuss the situation in greater detail. First of all, we use the symbol $\pi$, with deliberate ambiguity, to describe that norm on $F^{\prime} \otimes F$ as well as on $E^{\prime} \otimes E$. Furthermore, since $E^{\prime} \otimes E$ and $E \otimes E^{\prime}$ are obviously isomorphic to each other under the transposition transformation (see §3) that takes $x^{\prime} \otimes x$ into $x \otimes x^{\prime}$, we can also define a norm $\pi$ on $E \otimes E^{\prime}$ as that norm on $E \otimes E^{\prime}$ which renders the transposition transformation isometric. See the previous section for details.

Let us now turn to the products $S \times \mathrm{t}$ and $\mathrm{t} \times S$, defined in $\S 3$ (see relations (3.12) and (3.13) in that section), for $S \in \mathscr{L}\left(E, F^{\prime}\right)$ and $\mathrm{t} \in E \otimes F$. Since $S \times \mathrm{t}$ is contained in $E \otimes E^{\prime}$ and this latter space is a subspace of $E \otimes_{\pi} E^{\prime}$, we can, and will without further explanation, regard $S \times \mathrm{t}$ as an element of the complete tensor product $E \otimes_{\pi} E^{\prime}$. The next theorem will vindicate this choice. We shall follow a similar convention regarding the product $\mathrm{t} \times S$ and the spaces $F^{\prime} \otimes F$ and $F^{\prime} \otimes_{\pi} F$. Our generalization of the theorem of Schatten states that for every $S \in\left(E \otimes_{\alpha} F\right)^{\prime}$, the mapping $\mathrm{t} \rightarrow S \times \mathrm{t}$ of $E \otimes F$ into $E \otimes_{\pi} E^{\prime}$ can be extended from $E \otimes F$ to a bounded linear transformation of $E \otimes_{\alpha} F$ into $E \otimes_{\pi} E^{\prime}$. In fact, this property characterizes the elements of $\left(E \otimes_{\alpha} F\right)^{\prime}$ among those of $\mathscr{L}\left(E, F^{\prime}\right)$. We get a similar result for the product $t \times S$.

TheOREM 6.1. Let $S \in \mathscr{L}\left(E, F^{\prime}\right)$. Then $S$ belongs to $\left(E \otimes_{\alpha} F\right)^{\prime}$ if and only if the linear transformation $\mathrm{t} \rightarrow S \times \mathrm{t}$ of $E \otimes F$ into $E \otimes_{\pi} E^{\prime}$ can be extended to a bounded linear transformation of the entire $E \otimes_{\alpha} F$ into $E \otimes_{\pi} E^{\prime}$. In fact, there exists a constant $k_{1}>0$ so that we have, for every $S \in\left(E \otimes_{\alpha} F\right)^{\prime}$ and $t \in E \otimes_{\alpha} F$,

$$
\pi(S \times \mathrm{t}) \leqq k_{1} \alpha^{\prime}(S) \alpha(\mathrm{t})
$$

Also

$$
\langle\mathrm{t}, S\rangle=\operatorname{tr} S \times \mathrm{t} .
$$

Proof. We begin by establishing the relation (6.1) for $S \in\left(E \otimes_{\alpha} F\right)^{\prime}$ and $\mathrm{t}$ a finite tensor, i.e., an element of the algebraic tensor product $E \otimes F$. To do this we will need Theorem 4.2 and refer the reader to the relation (4.3) in that theorem. 
Let $\mathfrak{t} \in E \otimes F$ and $S \in\left(E \otimes_{\alpha} F\right)^{\prime}$. It follows from the definition of $\pi$ on $E \otimes E^{\prime}$ that

$$
\pi(S \times \mathfrak{t})=\sup \left|\operatorname{tr}\left[(S \times \mathfrak{t})^{\prime} \times T\right]\right|,
$$

where the sup is taken for all $T \in \mathscr{L} \#\left(E^{\prime}\right)$, such that $\|T\| \leqq 1$. Now $T=V^{\prime}$ for some $V \in \mathscr{L}(E)$, and we shall use this notation throughout this proof. The following relations are easily verified

$$
(S \times \mathfrak{t})^{\prime} \times T=\left(\mathfrak{t}^{\prime} \times S^{\prime}\right) \times T=\mathfrak{t}^{\prime} \times\left(T S^{\prime}\right)
$$

and

$$
\operatorname{tr}\left[(S \times \mathfrak{t})^{\prime} \times T\right]=\operatorname{tr}\left[\mathfrak{t}^{\prime} \times\left(T S^{\prime}\right)\right] .
$$

Now, since $T=V^{\prime}, T S^{\prime}=(S V)^{\prime}$ and we have

$$
\operatorname{tr}\left[(S \times \mathfrak{t})^{\prime} \times T\right]=\operatorname{tr}\left[\mathrm{t}^{\prime} \times(S V)^{\prime}\right]=\operatorname{tr}[(S V) \times \mathrm{t}] .
$$

Since $S \in\left(E \otimes_{\alpha} F\right)^{\prime}$ and the latter space is closed under right multiplications by elements of $\mathscr{L}(E)$, we have $S V \in\left(E \otimes_{\alpha} F\right)^{\prime}$ and

$$
\operatorname{tr}\left[(S \times \mathrm{t})^{\prime} \times T\right]=\operatorname{tr}[(S V) \times \mathrm{t}]=\langle\mathrm{t}, S V\rangle .
$$

Therefore, by Theorem 4.2,

$$
\begin{aligned}
\left|\operatorname{tr}\left[(S \times \mathfrak{t})^{\prime} \times T\right]\right| & =|\langle\mathfrak{t}, S V\rangle| \leqq \alpha^{\prime}(S V) \alpha(\mathfrak{t}) \\
& \leqq k_{1}\|V\| \alpha^{\prime}(S) \alpha(\mathfrak{t}) .
\end{aligned}
$$

From (6.3) and (6.8) we obtain (6.1) with the same $k_{1}$ as in (4.3). It follows also that, for fixed $S \in\left(E \otimes_{\alpha} F\right)^{\prime}$, the mapping $\mathrm{t} \rightarrow S \times \mathrm{t}$ of $E \otimes F$ into $E \otimes_{\pi} E^{\prime}$ can be extended to the entire $E \otimes_{\alpha} F$, satisfying (6.1). Now (6.2) is verified easily for finite tensors and extended by continuity to every $\mathrm{t} \in E \otimes_{\alpha} F$, with the help of (6.1).

Conversely, suppose that $S \in \mathscr{L}\left(E, F^{\prime}\right)$ is such that the mapping $\mathrm{t} \rightarrow S \times \mathrm{t}$ of $E \otimes F$ into $E \otimes_{\pi} E^{\prime}$ can be extended to the entire $E \otimes_{\alpha} F$, as a bounded linear transformation. We show that $S \in\left(E \otimes_{\alpha} F\right)^{\prime}$. But this is easy for, if $t \in E \otimes F$, we have

$$
|\langle\mathrm{t}, S\rangle|=|\operatorname{tr} S \times \mathrm{t}| \leqq \pi(S \times \mathrm{t}) \leqq \kappa \alpha(\mathrm{t}),
$$

where $\kappa$ is a bound for the transformation $t \rightarrow S \times t$. Since (6.9) holds for every finite tensor, it also holds for every $t \in E \otimes_{\alpha} F$. This completes our proof.

We have a companion theorem for the other product $t \times S$. We have chosen to state these two theorems separately in order not to confuse the situation. Their methods of proof are not identical, but they are sufficiently similar that an outline of the proof for the second theorem should be enough.

THeOREM 6.2. Let $S \in \mathscr{L}\left(E, F^{\prime}\right)$. Then $S$ belongs to $\left(E \otimes_{\alpha} F\right)^{\prime}$ if and only if the linear transformation $\mathrm{t} \rightarrow \mathrm{t} \times S$ of $E \otimes F$ into $F^{\prime} \otimes_{\pi} F$ can be extended to a bounded 
linear transformation of $E \otimes_{\alpha} F$ into $F^{\prime} \otimes_{\pi} F$. There exists a constant $k_{1}>0$ such that for every $\mathrm{t} \in E \otimes_{\alpha} F$ and $S \in\left(E \otimes_{\alpha} F\right)^{\prime}$ we have

$$
\pi(\mathrm{t} \times S) \leqq k_{1} \alpha^{\prime}(S) \alpha(\mathrm{t}) .
$$

Also

$$
\langle\mathrm{t}, S\rangle=\operatorname{tr} \mathrm{t} \times S .
$$

Proof. The proof of this theorem, as might be expected follows closely the pattern of the proof of the previous theorem. The only significant difference occurs in establishing (6.10) for finite tensors, where the necessary calculations differ from the ones in Theorem 6.1. We limit ourselves to this aspect of the proof. Let $\mathrm{t} \in E \otimes F$ and $S \in\left(E \otimes_{\alpha} F\right)^{\prime}$. As before, we have

$$
\pi(\mathrm{t} \times S)=\sup |\operatorname{tr}[(\mathrm{t} \times S) \times U]|,
$$

where the sup is taken for all $U \in \mathscr{L}^{\#}\left(F^{\prime}\right)$ with $\|U\| \leqq 1$. Now $(\mathrm{t} \times S) \times U=\mathrm{t} \times(U S)$, and since we are assuming that $\alpha$ is modular, $\left(E \otimes_{\alpha} F\right)^{\prime}$ is closed under left multiplication by elements of $\mathscr{L}^{\#}\left(F^{\prime}\right)$. Hence $U S \in\left(E \otimes_{\alpha} F\right)^{\prime}$. Then, again we have

$$
\operatorname{tr}[(\mathrm{t} \times S) \times U]=\operatorname{tr}[\mathrm{t} \times(U S)]=\langle\mathrm{t}, U S\rangle,
$$

and, by Theorem 4.2, we have

$$
\operatorname{tr}[(\mathrm{t} \times S) \times U] \leqq \alpha^{\prime}(U S) \alpha(\mathrm{t}) \leqq k_{1}\|U\| \alpha^{\prime}(S) \alpha(\mathrm{t}) .
$$

Now (6.14) and (6.12) yield (6.10) with the same $k_{1}$ as in Theorem 4.2. This completes our proof.

Briefly, Theorems 6.1 and 6.2 are to the effect that the products $S \times \mathrm{t}$ and $\mathrm{t} \times S$, defined originally for $S \in \mathscr{L}\left(E, F^{\prime}\right)$ and $\mathrm{t} \in E \otimes F$, now make sense for $S \in\left(E \otimes_{\alpha} F\right)^{\prime}$ and $\mathrm{t} \in E \otimes_{\alpha} F$, if $\alpha$ is modular, and that $S \times \mathrm{t} \in E \otimes_{\pi} E^{\prime}$ and $\mathrm{t} \times S \in F^{\prime} \otimes_{\pi} F$. The theorems, as they appear here, seem to be new, although there are several theorems in the literature which point towards them. There is the theorem of Schatten which we have quoted at the beginning of this section. There is also the result of Grothendieck [11, p. 84], stating that the product of a bounded linear transformation times a nuclear transformation is a nuclear transformation.

From the computations involved in the proof of Theorems 6.1 and 6.2, we can also establish the validity of the following corollary, whose details we leave to the reader. The importance of this corollary will become apparent soon. For the discussion of kernels of transformations we refer the reader to $\$ 3$.

CoROllaRY 6.1. Let $\mathrm{t} \in E \otimes_{\alpha} F$ and $S \in\left(E \otimes_{\alpha} F\right)^{\prime}$, then $S \times \mathrm{t}$ is a kernel of $S^{\prime} T_{\mathrm{t}}$ and $\mathrm{t} \times S$ a kernel of $T_{\mathrm{t}} S^{\prime}$.

We now turn the setting around to get, from the extensibility of the $x$-products, a characterization of modular norms.

THEOREM 6.3. Let $\alpha$ be a tensorial norm of $E \otimes F$. Then $\alpha$ is a modular norm if and only if, for every $S \in\left(E \otimes_{\alpha} F\right)^{\prime}$, the two linear transformations $\mathrm{t} \rightarrow S \times \mathrm{t}$ and 
$\mathrm{t} \rightarrow \mathrm{t} \times S$ of $E \otimes F$ into $E \otimes_{\pi} E^{\prime}$ and into $F^{\prime} \otimes_{\pi} F$, respectively, can be extended to bounded linear transformations on the entire $E \otimes_{\alpha} F$.

Proof. If $\alpha$ is modular, the desired extensibility is the contents of Theorems 6.1 and 6.2. Suppose conversely that the extensions in question are possible. We show that $\alpha$ is modular, i.e., for $S \in\left(E \otimes_{\alpha} F\right)^{\prime}, U \in \mathscr{L}^{\#}\left(F^{\prime}\right)$ and $V \in \mathscr{L}(E)$, both $U S$ and $S V$ belong to $\left(E \otimes_{\alpha} F\right)^{\prime}$. We do it only for $U S$. Fix $S$ and $U$. It suffices to show that the functional given by $U S$ is bounded, with respect to $\alpha$, on the algebraic tensor product $E \otimes F$. Now, by the extensibility property, there exists a $k>0$, such that $\pi(\mathrm{t} \times S) \leqq k \alpha(\mathrm{t})$. We now have (see (6.13) above)

$$
\langle\mathrm{t}, U S\rangle=\operatorname{tr}(\mathrm{t} \times U S)=\operatorname{tr}[(\mathrm{t} \times S) \times U] \text {. }
$$

Consequently $|\langle\mathrm{t}, U S\rangle| \leqq \pi(\mathrm{t} \times S)\|U\| \leqq k \alpha(\mathrm{t})\|U\|$, these relations following quickly from (6.15) and the definition (5.2) of $\pi$. This completes our discussion of the theorem.

Computations similar to those involved in deriving the relation (6.15) above yield the following associativity laws for the $\times$-products and the --product, the latter defined in $\S 4$. See $\S 5$ for the definition of $U^{\#}$, for $U \in \mathscr{L}^{\#}\left(F^{\prime}\right)$.

CoRollaRY 6.2. Let $\alpha$ be a modular norm of $E \otimes F, \mathrm{t} \in E \otimes_{\alpha} F, S \in\left(E \otimes_{\alpha} F\right)^{\prime}$, $U \in \mathscr{L}^{\#}\left(F^{\prime}\right)$, and $V \in \mathscr{L}(E)$. Then

$$
\begin{aligned}
\mathrm{t} \times(U S V)=(V \cdot \mathrm{t}) & \times(U S)=[(V \cdot \mathrm{t}) \times S] \times U, \\
(U S V) \times \mathrm{t} & =\left(\mathrm{t} \cdot U^{\#}\right) \times(S V) \\
& =V \times\left[S \times\left(\mathrm{t} \cdot U^{\#}\right)\right] .
\end{aligned}
$$

We now turn to another aspect of duality of modular cross norms. To prepare the ground we start by discussing duality in a general Banach space, which for the purpose of this discussion, we shall denote by $E$. We remind the reader of the concept of polar set [1, p. 51, Definition 2]. Let $C$ be a subset of $E$. The polar set $C^{\circ}$ is the subset of $E^{\prime}$ consisting of all $x^{\prime} \in E^{\prime}$ such that

$$
\left|\left\langle x, x^{\prime}\right\rangle\right| \leqq 1 \text {, }
$$

for every $x \in C$. For $D \subset E^{\prime}$ we shall talk of the polar set $D^{-}$of $D$ in $E$ (not in $E^{\prime \prime}$ ) to be the set of all $x \in E$ which satisfy (6.18) for every $x^{\prime} \in D$. For $C \subset E$, the double polar $C^{\circ-}=\left(C^{\circ}\right)^{-}$is again a subset of $E$, which contains $C$. Similarly for $D \subset E^{\prime}$, and the double polar $\left(D^{-}\right)^{\circ}$. We shall have occasion to use the double polar theorem, which states that for every $C \subset E,\left(C^{\circ}\right)^{-}$is the smallest closed convex symmetric-i.e., équilibré-subset of $E$ containing $C$ and that for every $D \subset E^{\prime},\left(D^{-}\right)^{\circ}$ is the smallest weakly* closed, convex, symmetric subset of $E^{\prime}$ containing $D$. This theorem can be found stated and proved in [1, Proposition 3, p. 52]. In the particular case in which $C$ is a vector space, $C^{\circ}$ is what is normally called the annihilator of $C$, namely the set of all $x^{\prime} \in E^{\prime}$ such that $\left\langle x, x^{\prime}\right\rangle=0$, for every $x \in C$. Similarly for a vector subspace of $E^{\prime}$. 
We have already described the dual space $\left(E \otimes_{\alpha} F\right)^{\prime}$ of $E \otimes_{\alpha} F$ as a left module over $\mathscr{L}^{\#}\left(F^{\prime}\right)$ and as a right module over $\mathscr{L}(E)$ by means of the trilinear expression $U S V, U \in \mathscr{L}^{\#}\left(F^{\prime}\right), S \in\left(E \otimes_{\alpha} F\right)^{\prime}$ and $V \in \mathscr{L}(E)$, which is ordinary composition of operators. We have also seen that, as a result of Lemma 4.1, we can define a structure of left module over $\mathscr{L}(E)$ and of right module over $\mathscr{L}(F)$ on the tensor product $E \otimes_{\alpha} F$ itself, under the trilinear mapping $V \cdot \mathrm{t} \cdot W$, for $V \in \mathscr{L}(E)$, $\mathrm{t} \in E \otimes_{\alpha} F$ and $W \in \mathscr{L}(F)$. This trilinear mapping is defined by equations (4.11) and (4.12). In the next theorem we are going to see that the structure of module of $E \otimes_{\alpha} F$ and that of $\left(E \otimes_{\alpha} F\right)^{\prime}$ can be put into a relation of duality by means of the bilinear pairing $\left[E \otimes_{\alpha} F\right] \times\left[\left(E \otimes_{\alpha} F\right)^{\prime}\right] \rightarrow E \otimes_{\pi} E^{\prime}$ defined by the product $S \times \mathrm{t}$, just obtained in Theorem 6.1 and by the pairing corresponding to the product $\mathrm{t} \times S$ in Theorem 6.2. In the proof we shall need two relations which we obtain now. Let $U \in \mathscr{L}^{\#}\left(F^{\prime}\right)$. Then there exists $W \in \mathscr{L}(F)$ such that $U=W^{\prime}$. Likewise for a $T \in \mathscr{L}^{\#}\left(E^{\prime}\right)$ and a corresponding $V \in \mathscr{L}(E)$. Throughout the discussion of the theorem we shall use this notation consistently. Let now $\mathrm{t} \in E \otimes_{\alpha} F$ and $S \in\left(E \otimes_{\alpha} F\right)^{\prime}$. We have seen (Theorem 6.1) that $S \times \mathrm{t} \in E \otimes_{\pi} E^{\prime}$ and that (Theorem 6.2) the tensor $\mathrm{t} \times S$ belongs to $F^{\prime} \otimes_{\pi} F$. We have also seen (Theorem 5.3) that $U \times(\mathrm{t} \times S)$ is well defined, as well as (Theorem 5.2) $(\mathrm{t} \times S) \cdot W$, and $\mathrm{t} \cdot W$ (by Theorem 4.6). Similarly, we have the well-defined expressions $T \times(S \times \mathrm{t})^{\prime}$ and $S \times(\mathrm{t} \cdot V)$. The following relations can be verified by simple algebraic manipulations for finite tensors and, following the usual procedure, extended to infinite tensors in $E \otimes_{\alpha} F$ by a continuity argument.

$$
\begin{gathered}
U \times(\mathrm{t} \times S)=(\mathrm{t} \times S) \cdot W=(\mathrm{t} \cdot W) \times S, \\
\operatorname{tr}\left[T \times(S \times \mathrm{t})^{\prime}\right]=\operatorname{tr}[S \times(V \cdot \mathrm{t})]=\operatorname{tr}(S V) \times \mathrm{t} .
\end{gathered}
$$

THEOREM 6.4. The polar set of an $\mathscr{L}(E)$-left $(\mathscr{L}(F)$-right $)$ submodule $\mathscr{M}$ of $E \otimes_{\alpha} F$ consists of all the left (right) annihilators of $\mathscr{M}$ in $\left(E \otimes_{\alpha} F\right)^{\prime}$, under the $\times$-product, that is $\mathscr{M}^{\circ}$ consists of all $S \in\left(E \otimes_{\alpha} F\right)^{\prime}$ such that $S \times \mathrm{t}=0,(\mathrm{t} \times S=0)$ for every $t \in \mathscr{M}$. Consequently, the polar set of a two-sided submodule of $E \otimes_{\alpha} F$ under its $\mathscr{L}(E)$-left and $\mathscr{L}(F)$-right structure, consists of all two-sided annihilators of the submodule, under the $\times$-product.

Proof (for left submodules). Let $S \in\left(E \otimes_{\alpha} F\right)^{\prime}$. If $S \times \mathrm{t}=0$ for every $\mathrm{t} \in \mathscr{M}$, it is clear from $\langle\mathrm{t}, S\rangle=\operatorname{tr} S \times \mathrm{t}$, that $S \in \mathscr{M}^{\circ}$. Hence the left annihilator of $\mathscr{M}$ is contained in $\mathscr{M}^{\circ}$. We establish the reverse inclusion. Let $S \in \mathscr{M}^{\circ}$ and $\mathrm{t} \in \mathscr{M}$. We show $S \times \mathrm{t}=0$. Now $S \times \mathfrak{t} \in E \otimes_{\pi} E^{\prime}$. So let $T \in \mathscr{L}^{\#}\left(E^{\prime}\right)$. Then

$$
\langle S \times \mathrm{t}, T\rangle=\operatorname{tr}\left[T \times(S \times \mathrm{t})^{\prime}\right]=\operatorname{tr}[S \times(V \cdot \mathrm{t})] .
$$

The relation (6.21) follows from (6.20) above. Now, since $\mathscr{M}$ is a left submodule, $V \cdot \mathrm{t} \in \mathscr{M}$, and the extreme right expression in (6.21) vanishes for every $T \in \mathscr{L}^{\#}\left(E^{\prime}\right)$. From this we conclude that $S \times \mathrm{t}=0$ for every $\mathrm{t} \in \mathscr{M}$, which was to be established. 
A completely analogous argument, which we omit, yields the following corresponding theorem for submodules of $\left(E \otimes_{\alpha} F\right)^{\prime}$.

THEOREM 6.5. The polar set in $E \otimes_{\alpha} F$ of an $\mathscr{L}^{\#}\left(F^{\prime}\right)$-left $(\mathscr{L}(E)$-right $)$ submodule of $\left(E \otimes_{\alpha} F\right)^{\prime}$ is its left (right) annihilators in $E \otimes_{\alpha} F$ under the $\times$-product. Consequently, the polar set of a two-sided submodule of $\left(E \otimes_{\alpha} F\right)^{\prime}$ under its $\mathscr{L}^{\#}\left(F^{\prime}\right)$-left, $\mathscr{L}(E)$-right structure consists of all its two-sided annihilators under the $\times$-product.

COROLlaRY 6.3. The polar set of an $\mathscr{L}(E)$-left $(\mathscr{L}(F)$-right) submodule of $E \otimes_{\alpha} F$ is an $\mathscr{L}(E)$-right $\left(\mathscr{L}^{\#}\left(F^{\prime}\right)\right.$-left $)$ submodule of $\left(E \otimes_{\alpha} F\right)^{\prime}$.

Proof (for $\mathscr{L}(F)$-right submodules). Let $\mathscr{M}$ be an $\mathscr{L}(F)$-right submodule of $E \otimes_{\alpha} F$. We show that the polar $\mathscr{M}^{\circ}$ is an $\mathscr{L}^{\#}\left(F^{\prime}\right)$-left submodule of $(E \otimes F)^{\prime}$. Let $S \in \mathscr{M}^{\circ}$ and $U \in \mathscr{L}^{\#}\left(F^{\prime}\right)$. It suffices, by Theorem 6.2, to show that $U S$ annihilates $\mathscr{M}$ on the right. This follows from the easily verified relation,

$$
\mathrm{t} \times(U S)=(\mathrm{t} \times S) \times U,
$$

for every $\mathrm{t} \in E \otimes_{\alpha} F$. This completes our proof.

We now have the companion corollary for submodules of $\left(E \otimes_{\alpha} F\right)^{\prime}$, having an anologous proof, which we omit.

Corollary 6.4. The polar set in $E \otimes_{\alpha} F$ of an $\mathscr{L}^{\#}\left(F^{\prime}\right)$-left $(\mathscr{L}(E)$-right $)$ submodule of $\left(E \otimes_{\alpha} F\right)^{\prime}$ is an $\mathscr{L}(F)$-right $(\mathscr{L}(E)$-left $)$ submodule of $E \otimes_{\alpha} F$.

COROLlaRY 6.5. The weak* closure of an $\mathscr{L}^{\#}\left(F^{\prime}\right)$-left $(\mathscr{L}(E)$-right $)$ submodule of $\left(E \otimes_{\alpha} F\right)^{\prime}$ is an $\mathscr{L}^{\#}\left(F^{\prime}\right)$-left $(\mathscr{L}(E)$-right $)$ submodule of $\left(E \otimes_{\alpha} F\right)^{\prime}$.

Proof (for $\mathscr{L}^{\#}\left(F^{\prime}\right)$-left submodules). Let $\mathscr{M}$ be an $\mathscr{L}^{\#}\left(F^{\prime}\right)$-left submodule of $\left(E \otimes_{\alpha} F\right)^{\prime}$ and $\overline{\mathscr{M}}$ the weak* closure of $\mathscr{M}$. By the double polar theorem quoted earlier in this section, $\overline{\mathscr{M}}=\left(\mathscr{M}^{-}\right)^{\circ}$. By Corollary $6.4, \mathscr{M}^{-}$is an $\mathscr{L}(F)$-right submodule of $E \otimes_{\alpha} F$. By Corollary 6.3, $\overline{\mathscr{M}}$ is an $\mathscr{L}^{\#}\left(F^{\prime}\right)$-left submodule of $\left(E \otimes_{\alpha} F\right)^{\prime}$.

Q.E.D.

For two-sided submodules we adopt new conventions. For instance, by an $\mathscr{L}^{\#}\left(F^{\prime}\right)-\mathscr{L}(E)$-submodule of $\left(E \otimes_{\alpha} F\right)^{\prime}$ we shall mean a vector subspace of $\left(E \otimes_{\alpha} F\right)^{\prime}$ which is both an $\mathscr{L}^{\#}\left(F^{\prime}\right)$-left and an $\mathscr{L}(E)$-right submodule of $\left(E \otimes_{\alpha} F\right)^{\prime}$. Similar concepts follow similar conventions.

CoROllary 6.6. The polar set (polar set in $E \otimes_{\alpha} F$ ) of an $\mathscr{L}(E)-\mathscr{L}(F)$-submodule of $E \otimes_{\alpha} F\left(\mathscr{L}^{\#}\left(F^{\prime}\right)-\mathscr{L}(E)\right.$-submodule of $\left.\left(E \otimes_{\alpha} F\right)^{\prime}\right)$ is an $\mathscr{L}^{\#}\left(F^{\prime}\right)-\mathscr{L}(E)-$ submodule of $\left(E \otimes_{\alpha} F\right)^{\prime}\left(\right.$ an $\mathscr{L}(E)-\mathscr{L}(F)$-submodule of $\left.E \otimes_{\alpha} F\right)$.

Let us consider strongly modular cross norms. We shall see that in the same manner that the entire space $\mathscr{L}\left(F^{\prime}\right)$ replaces $\mathscr{L}^{\#}\left(F^{\prime}\right)$ in the definition, the norm $\gamma$ replaces $\pi$ in the theorems. The proof of the following theorem is an exact analogue of that of Theorem 6.2, and will be omitted. For the definition of the symbol $\Phi_{\beta}^{\alpha}$ see $\S 3$. 
THEOREM 6.6. Let $\alpha$ be a strongly modular norm of $E \otimes F$ (for a definition, see $\S 4)$, and $S \in \mathscr{L}\left(E, F^{\prime}\right)$. Then $S \in\left(E \otimes_{\alpha} F\right)^{\prime}$ if and only if the mapping $\mathrm{t} \rightarrow \mathrm{t} \times S$ of $E \otimes F$ into $F^{\prime} \otimes F \subset F^{\prime} \otimes_{\gamma} F$ can be extended to a bounded linear transformation $\mathrm{t} \rightarrow \mathrm{t} \square S$ of $E \otimes_{\alpha} F$ into $F^{\prime} \otimes_{\gamma} F$, such that

$$
\Phi_{\pi}^{\gamma}(\mathfrak{t} \times S)=\mathfrak{t} \times S .
$$

The bilinear pairing represented by the expression $\mathrm{t} \square S$, for $\mathrm{t} \in E \otimes_{\alpha} F$ and $S \in\left(E \otimes_{\alpha} F\right)^{\prime}$ is a continuous bilinear mapping of $\left(E \otimes_{\alpha} F\right) \times\left[\left(E \otimes_{\alpha} F\right)^{\prime}\right]$ into $F^{\prime} \otimes_{\gamma} F$.

Now the analogues of Theorem 6.4 and of Corollaries 6.1 through 6.6 for strongly modular norms can be stated and proved. We shall not give the details, but will make use of the results when they are needed. The results and the proofs are obtained by the simple formal device of replacing in a systematic way in each of the old statements the space $\mathscr{L}^{\#}\left(F^{\prime}\right)$ by $\mathscr{L}\left(F^{\prime}\right)$, the norm $\pi$ by $\gamma$ and the $\times-$ product by the $\square$-product. Perhaps it might be well to state explicitly, though without proof, the appropriate counterpart of Theorem 6.3.

THEOREM 6.7. Let $\alpha$ be a modular norm of $E \otimes F$. Then $\alpha$ is strongly modular if and only if the $\times$-product $\mathrm{t} \times S$, for $\mathrm{t} \in E \otimes F$ and $S \in\left(E \otimes_{\alpha} F\right)^{\prime}$, can be extended to a product $\mathrm{t} \square S \in F^{\prime} \otimes_{\gamma} F$, for $\mathrm{t} \in E \otimes_{\alpha} F$ and $S \in\left(E \otimes_{\alpha} F\right)^{\prime}$, in such a way that, for each fixed $S \in\left(E \otimes_{\alpha} F\right)^{\prime}$, the mapping $\mathrm{t} \rightarrow \mathrm{t} \square S$ of $E \otimes_{\alpha} F$ into $F^{\prime} \otimes_{\gamma} F$ is continuous.

However, for strongly modular norms, we can give a considerably stronger form of all these theorems, under the additional assumption that $F$ satisfies the condition of approximation of Grothendieck (see $\S 1$ for a discussion of this condition). In the theory presented in this section up to this point we have endeavored to give simple characterizations of the various relevant objects, such as the individual elements of $\left(E \otimes_{\alpha} F\right)^{\prime}$. One inherent weakness is that, while the concepts of modularity and strong modularity have purely algebraic definitions, the characterizations in question have topological elements, since they involve, for instance, the boundedness of the transformations $\mathrm{t} \rightarrow S \times \mathrm{t}$ and $\mathrm{t} \rightarrow \mathrm{t} \times S$. The purpose of the next few theorems is to replace the boundedness condition by an algebraic property, namely that embodied in Corollary 6.1 above.

Suppose now that $F$ satisfies the condition of approximation of Grothendieck. This implies that the natural mapping (see $\S 3) \Psi^{\gamma \gamma}$ of $F^{\prime} \otimes_{\gamma} F$ into $\mathscr{L}(F)$ is isomorphic, i.e., it defines a one to one transformation between the tensors of $F^{\prime} \otimes_{\gamma} F$ and the operators in $\mathscr{L}(F)$ of which they are kernels.

THEOREM 6.8. Suppose that $F$ satisfies the condition of approximation of Grothendieck and that $\alpha$ is an admissible (§3) norm of $E \otimes F$. Let $S \in \mathscr{L}\left(E, F^{\prime}\right)$. If the linear transformation $\mathrm{t} \rightarrow \mathrm{t} \times S$ of $E \otimes F$ into $F^{\prime} \otimes_{\gamma} F$ can be extended to a linear transformation $\mathrm{t} \rightarrow \mathrm{t} \square S$ of $E \otimes_{\alpha} F$ into $F^{\prime} \otimes_{\gamma} F$ in such a way that, for every $\mathrm{t} \in E \otimes_{\alpha} F$, 
$\mathrm{t} \square S$ is the $\operatorname{kernel}\left({ }^{9}\right)$ of $T_{\mathrm{t}} S^{\prime}$, under the natural image $F^{\prime} \otimes_{\gamma} F$ into $\mathscr{L}(F)$, then $S \in\left(E \otimes_{\alpha} F\right)^{\prime}$ and the transformation $\mathrm{t} \rightarrow \mathrm{t} \square S$ is bounded.

Proof. Let us assume that $\mathrm{t} \rightarrow \mathrm{t} \times S$ can be extended to a linear transformation $\mathfrak{t} \rightarrow \mathfrak{t} \square S$ of $E \otimes_{\alpha} F$ into $F^{\prime} \otimes_{\gamma} F$, with the required kernel properties. Using the closed-graph theorem, we see that it suffices to prove that, if $t_{1}, t_{2}, \cdots$ is a sequence of tensors in $E \otimes_{\alpha} F$ converging to a tensor $\mathrm{t}_{0} \in E \otimes_{\alpha} F$, and if $\mathrm{t}_{1} \square S, \mathrm{t}_{2} \square S, \cdots$ converges to a tensor $\mathfrak{w}_{0} \in F^{\prime} \otimes_{\gamma} F$, then $\mathrm{t}_{0} \square S=\mathfrak{w}_{0}$. Since $F$ is assumed to satisfy the condition of approximation of Grothendieck, one simply has to show that $\mathrm{t}_{0} \square S$ and $\mathfrak{w}_{0}$ are kernels of the same operator in $\mathscr{L}(F)$. Let us now introduce some notation. Let each $\mathrm{t}_{n}$ be a kernel of a transformation $T_{n}: E^{\prime} \rightarrow F$, for $n=0,1,2, \cdots$, and each $\mathrm{t}_{n} \square S$ the kernel of an operator $W_{n} \in \mathscr{L}(F)$. Finally, suppose that $\mathfrak{w}_{0}$ is the kernel of $W_{0} \in \mathscr{L}(F)$. By hypothesis we have

$$
W_{n}=T_{n} S^{\prime} .
$$

Our task is then to show that $W_{0}=T_{0} S^{\prime}$, i.e.,

$$
\left\langle W_{0} y, y^{\prime}\right\rangle=\left\langle T_{0} S^{\prime} y, y^{\prime}\right\rangle,
$$

for every $y \in F$ and $y^{\prime} \in F^{\prime}$. We shall consider the variable tensor $y \otimes y^{\prime} \in F \otimes F^{\prime}$ to be the kernel of a variable operator $U \in \mathscr{L}\left(F^{\prime}\right)=\left(F^{\prime} \otimes_{\gamma} F\right)^{\prime}$. We then have, by starting with the left-hand member of (6.25), (see (3.18))

$$
\begin{aligned}
\left\langle W_{0} y, y^{\prime}\right\rangle & =\left\langle\mathfrak{w}_{0}, U\right\rangle=\lim _{n \rightarrow \infty}\left\langle\mathrm{t}_{n} \times S, U\right\rangle=\lim _{n \rightarrow \infty}\left\langle W_{n} y, y^{\prime}\right\rangle \\
& =\lim _{n \rightarrow \infty}\left\langle T_{n} S^{\prime} y, y^{\prime}\right\rangle .
\end{aligned}
$$

The last equality in (6.26) follows from (6.24). Let us now consider the tensor $S^{\prime} y \otimes y^{\prime} \in E^{\prime} \otimes F^{\prime}$. It is easy to check that this tensor is the kernel of the linear transformation $U S$ which belongs to $\left(E \otimes_{\alpha} F\right)^{\prime}$, since $\alpha$ is assumed to be admissible. Our conclusion follows as we now indicate. We have $\left\langle T_{n} S^{\prime} y, y^{\prime}\right\rangle=\left\langle\mathrm{t}_{n}, U S\right\rangle$ for every $n$. Hence,

$$
\begin{aligned}
\left\langle W_{0} y, y^{\prime}\right\rangle & =\lim _{n \rightarrow \infty}\left\langle T_{n} S^{\prime} y, y^{\prime}\right\rangle=\lim _{n \rightarrow \infty}\left\langle\mathrm{t}_{n}, U S\right\rangle=\left\langle\mathrm{t}_{0}, U S\right\rangle \\
& =\left\langle\mathrm{t}_{0} \square S, U\right\rangle=\left\langle T_{0} S^{\prime} y, y^{\prime}\right\rangle .
\end{aligned}
$$

We have thus established (6.25), and the conclusion of the theorem.

Now the following two theorems, given without proof, follow easily from Theorem 6.8 and earlier analogous theorems.

THEOREM 6.9. Suppose that $F$ satisfies the condition of approximation of Grothendieck and that $\alpha$ is a strongly modular norm of $E \otimes F$. Let $S \in \mathscr{L}\left(E, F^{\prime}\right)$. Then

${ }^{(9)}$ The choice between the phrases a kernel and the kernel is deliberate, and is meant to indicate whether it is assumed (or it follows) that the modular norm of the tensor product in question is faithful. 
$S \in\left(E \otimes_{\alpha} F\right)^{\prime}$ if and only if the linear transformation $\mathrm{t} \rightarrow \mathrm{t} \times S$ of $E \otimes F$ into $F^{\prime} \otimes_{\gamma} F$ can be extended to a linear transformation $\mathrm{t} \rightarrow \mathrm{t} \square S$ of $E \otimes_{\alpha} F$ into $F^{\prime} \otimes_{\gamma} F$ such that, for every $\mathrm{t} \in E \otimes_{\alpha} F, \mathrm{t} \square S$ is the kernel of $T_{\mathrm{t}} S^{\prime}$.

THEOREM 6.10. Suppose that $F$ satisfies the condition of approximation of Grothendieck, and that $\alpha$ is a modular norm of $E \otimes F$. Then $\alpha$ is strongly modular if and only if, for every $S \in\left(E \otimes_{\alpha} F\right)^{\prime}$, the linear transformation $\mathrm{t} \rightarrow \mathrm{t} \times S$ of $E \otimes F$ into $F^{\prime} \otimes_{\gamma} F$ can be extended to a linear transformation $\mathrm{t} \rightarrow \mathrm{t} \square S$ of $E \otimes_{\alpha} F$ into $F^{\prime} \otimes_{\gamma} F$ in such a way that, for every $\mathrm{t} \in E \otimes_{\alpha} F$ and $S \in\left(E \otimes_{\alpha} F\right)^{\prime}, \mathrm{t} \square S$ is the kernel of $T_{\mathrm{t}} S^{\prime}$.

We do not know if there are any modular norms that are not strongly modular, but we have the following theorem. For a discussion of the possible equivalence of the norm $\pi$ and the norm $\gamma$, see Theorem 5.4 in the previous section.

THEOREM 6.11. Suppose that the norm $\pi$ on $F^{\prime} \otimes F$ is equivalent to the norm $\gamma$ on $F^{\prime} \otimes F$. Then every modular norm of $E \otimes F$ is strongly modular.

Proof. This follows easily from Theorem 6.10. Since $\pi$ on $F^{\prime} \otimes F$ is equivalent to $\gamma$, we have that $F^{\prime} \otimes_{\pi} F=F^{\prime} \otimes_{\gamma} F$. Now let $\alpha$ be a modular norm of $E \otimes F$. Then, by Theorem 6.2, for every $S \in\left(E \otimes_{\alpha} F\right)^{\prime}$, the mapping $\mathrm{t} \rightarrow \mathrm{t} \times S$ from $E \otimes F$ into $F^{\prime} \otimes F$ can be extended to a bounded linear transformation of $E \otimes_{\alpha} F$ into $F^{\prime} \otimes_{\pi} F$, hence into $F^{\prime} \otimes_{\gamma} F$. Our conclusion now follows directly from Theorem 6.10.

We shall need the next theorem for our discussion of the norms $\lambda$ and $\gamma$, as well as for parts of the general theory presented in $\$ 9$, below. We shall need to compute with the space $E \otimes_{\gamma} E^{\prime}$. According to equation (3.16) the dual space of $E \otimes_{\gamma} E^{\prime}$ is $\mathscr{L}\left(E, E^{\prime \prime}\right)$. However one can see easily that this space is isometrically isomorphic to the algebra $\mathscr{L}\left(E^{\prime}\right)$, the isomorphism, being implemented by the mapping $T \rightarrow T^{\#}$, for $T \in \mathscr{L}\left(E^{\prime}\right)$, where $T^{\#}$ is the restriction to $E$ of the transpose $T^{\prime}$. Consequently, we are going to consider $\mathscr{L}\left(E^{\prime}\right)$ as the dual space of $E \otimes E^{\prime}$. In doing so one has to realize that the action of a $T \in \mathscr{L}\left(E^{\prime}\right)$ on $\mathrm{t} \in E \otimes_{\gamma} E^{\prime}$ now takes the form

$$
\langle\mathfrak{t}, T\rangle=\operatorname{tr}\left(\mathfrak{t}^{\prime} \times T\right) .
$$

Once one establishes that the various operations in (6.28) make sense, one establishes the relation itself easily by simple algebraic manipulations. Thus we now simply justify the operations involved. In the first place, the transposition of tensors, defined for finite tensors in $\S 3$, gives an isometric mapping of $E \otimes E^{\prime}$ onto $E^{\prime} \otimes E$, both spaces with the greatest cross norm $\gamma$, hence can be extended to an isometric mapping, still indicated by $\mathrm{t} \rightarrow \mathrm{t}^{\prime}$ of $E \otimes_{\gamma} E^{\prime}$ onto $E^{\prime} \otimes_{\gamma} E$. Now, since $\gamma$ is strongly modular, $T \in \mathscr{L}\left(E^{\prime}\right)$ and $\mathrm{t}^{\prime} \in E^{\prime} \otimes_{\gamma} E$, it follows from Theorem 6.7 that the product $\mathrm{t}^{\prime} \square T \in E^{\prime} \otimes_{\gamma} E$ is well defined. 
THEOREM 6.12. Let $\alpha$ be a norm of $E \otimes F$ which can be extended to a modular norm of $E^{\prime \prime} \otimes F \supset E \otimes F$. Then $\alpha$ is modular on $E \otimes F$, and, for every $S \in\left(E \otimes_{\alpha} F\right)^{\prime}$, the mapping $\mathrm{t} \rightarrow S \times \mathrm{t}$ of $E \otimes F$ into $E \otimes E^{\prime} \subset E \otimes_{\gamma} E^{\prime}$ can be extended to a bounded linear transformation $\mathrm{t} \rightarrow S \square \mathrm{t}$ of $E \otimes_{\alpha} F$ into $E \otimes_{\gamma} E^{\prime}$, such that $S \square \mathrm{t}$ is a kernel of $S^{\prime} T_{\mathfrak{t}}$ and

$$
\langle\mathrm{t}, S\rangle=\operatorname{tr}(S \square \mathrm{t}) .
$$

Proof. $\alpha$ is obviously modular on $E \otimes F$. The crux of the matter here is to prove that the mapping $\mathrm{t} \rightarrow S \times \mathrm{t}$ of $E \otimes F$ into $E \otimes E^{\prime}$ is bounded with respect to $\alpha$ and $\gamma$. The rest of the proof, which we omit, follows the familiar pattern established in the proof of Theorem 6.1. So, let $\mathrm{t} \in E \otimes F$. Since $S \times \mathrm{t} \in E \otimes E^{\prime} \subset E \otimes_{\gamma} E^{\prime}$, it follows from the discussion preceding this theorem (see relation (6.28)) that there exists $T \in \mathscr{L}\left(E^{\prime}\right),\|T\| \leqq 1$, such that $\gamma(S \times \mathfrak{t})=\operatorname{tr}\left[(S \times \mathfrak{t})^{\prime} \times T\right]$. Since we are dealing with finite tensors, all manipulations involved are purely algebraic. We see then that

$$
(S \times \mathfrak{t})^{\prime} \times T=\left(S T^{\prime}\right)^{\prime} \times \mathfrak{t} .
$$

Now we use the fact that $\alpha$ extends to a modular norm of $E^{\prime \prime} \otimes F$, which we denote again by $\alpha$. This tells us that $S$, as a bounded linear functional, can be extended to a bounded linear functional on $E^{\prime \prime} \otimes_{\alpha} F$, which we continue to denote $S$. This new linear functional is also a transformation belonging to $\mathscr{L}\left(E^{\prime \prime}, F^{\prime}\right)$. It is not very hard to see that such a transformation extends to $E^{\prime \prime}$ the transformation $S: E \rightarrow F^{\prime}$, which represented the original functional. We also know from the Hahn-Banach theorem that the extension involved can be made in such a way as to preserve the norm of the functionals. We call $\alpha^{\prime}$ the dual norm of $\alpha$ on $E^{\prime \prime} \otimes F$. With these conventions, we can consider $S$ and $S T^{\prime}$ in (6.30) as elements of $\left(E^{\prime \prime} \otimes_{\alpha} F\right)^{\prime}$ $\subset \mathscr{L}\left(E^{\prime \prime}, F^{\prime}\right)$. Since $\alpha$ is modular on $E^{\prime \prime} \otimes F$, we have that $S T^{\prime} \in\left(E^{\prime \prime} \otimes_{\alpha} F\right)^{\prime}$ and then

$$
\gamma(S \times \mathfrak{t})=\operatorname{tr}\left[\left(S T^{\prime}\right) \times \mathfrak{t}\right] \leqq \alpha^{\prime}\left(S T^{\prime}\right) \alpha(\mathrm{t}) \leqq k_{1} \alpha^{\prime}(S) \alpha(\mathrm{t}) .
$$

The last inequality in (6.31) follows from Theorem 4.2. This completes our proof.

If the hypothesis on $\alpha$ in Theorem 6.12 seems somewhat artificial, it must be kept in mind that it is obviously satisfied by any modular norm $\alpha$ on $E \otimes F$, provided $E$ is reflexive. It is also clearly satisfied for $\alpha=\lambda$, whether $E$ is reflexive or not. This is because $\lambda$ is really the operator norm. It is not so immediate that the hypothesis is also satisfied for $\alpha=\gamma$, and general $E$, but it is true. This follows from a theorem of Grothendieck, which states that the natural mapping of $E \otimes F$ into $E^{\prime \prime} \otimes F$, can be extended to an isometry of $E \otimes_{\gamma} F$ into $E^{\prime \prime} \otimes_{\gamma} F$. This theorem is very natural, but nontrivial. If the reader doubts it, he should attempt proving it, and then examine the pitfalls that are connected with it as pointed out by Schatten [14, Chapter III, §7]. The proof of the theorem of Grothendieck appears in [11, p. 41, Corollary 3]. We thus have that, since $\gamma$ is strongly modular and satisfies the 
hypothesis of Theorem 6.12 , both the products $\mathrm{t} \square S \in F^{\prime} \otimes_{\gamma} F$ and $S \square \mathrm{t} \in E \otimes_{\gamma} E^{\prime}$ are well defined.

In view of Theorem 6.12 we can easily formulate and prove theorems concerning submodules and annihilators in relation to the product $S \square \mathrm{t}$, analogous to those given earlier in this section for the $\times$-products and for the product $t \square S$. However we omit the details, although we will use the results freely, when necessary, as if they had been spelled out in detail.

Let us again consider the norm $\lambda$ on $E \otimes F$. It was shown by Grothendieck [11, Theorem 3, p. 100] that every transformation $S \in\left(E \otimes_{\lambda} F\right)^{\prime}$ is characterized by a representation

$$
S=\int_{\Sigma} S_{x^{\prime} \otimes y^{\prime}} d \mu
$$

Therefore he called an element of $\left(E \otimes_{\lambda} F\right)^{\prime}$ an integral transformation. The compact Hausdorff space $\Sigma$ is the cartesian product of the solid unit spheres of $E^{\prime}$ and $F^{\prime}$ with their weak* topologies, and $\mu$ is a positive Radon measure on $\Sigma$ such that $\mu(\Sigma)=\lambda^{\prime}(S)$. For each $\left(x^{\prime}, y^{\prime}\right) \in \Sigma, x^{\prime} \otimes y^{\prime}$ is a kernel of an element $S_{x^{\prime} \otimes y^{\prime}} \in\left(E \otimes_{\lambda} F\right)^{\prime}$, and the mapping $\left(x^{\prime}, y^{\prime}\right) \rightarrow S_{x^{\prime} \otimes y^{\prime}}$ of $\Sigma$ into the Banach space $\left(E \otimes_{\lambda} F\right)^{\prime}$ is continuous. Hence the integral in (6.32) can be interpreted in the sense of Bochner [12, Chapter III]. We have seen that $\lambda$ on $E \otimes F$ is strongly modular and satisfies the hypothesis of Theorem 6.12. Therefore, for $\mathrm{t} \in E \otimes_{\lambda} F$ and $S \in\left(E \otimes_{\lambda} F\right)^{\prime}$, the two products $\mathrm{t} \square S \in F^{\prime} \otimes_{\gamma} F$ and $S \square \mathrm{t} \in E \otimes_{\gamma} E^{\prime}$ are well defined. This result is essentially due to Grothendieck [11, Theorem 10, p. 132]. From formula (6.32) we can now give integral representations for these products, as follows.

$$
\begin{aligned}
& \mathfrak{t} \square S=\int_{\Sigma}\left(y^{\prime} \otimes T_{\mathfrak{i}} x^{\prime}\right) d \mu, \\
& S \square \mathfrak{t}=\int_{\Sigma}\left(T_{\mathrm{t}}^{\prime} y^{\prime} \otimes x^{\prime}\right) d \mu .
\end{aligned}
$$

7. Uniform and ordinary norms. We have defined a norm $\alpha$ on $E \otimes F$ to be uniform if

$$
\alpha[V \otimes W(\mathfrak{t})] \leqq\|V\|\|W\| \alpha(\mathfrak{t}),
$$

for every $V \in \mathscr{L}(E), W \in \mathscr{L}(F)$, and $\mathrm{t} \in E \otimes F$; and to be ordinary if

$$
\lambda \leqq \alpha \leqq \gamma
$$

Evidently (7.1) holds for every $\mathrm{t} \in E \otimes_{\alpha} F$ as well. An ordinary norm is of necessity a cross norm. We have already explained the origin of uniform cross norms in the work of Schatten and their role in our own work on tensor products of Banach algebras. The main purpose of the present section is to show that every modular norm is equivalent to a uniform norm and has all the essential topological properties 
normally attributed to ordinary norms. Therefore any of the known results of topological character involving uniform cross norms or ordinary norms extends to modular norms.

THEOREM 7.1. Let $\alpha$ be a norm of $E \otimes F$. Then $\alpha$ is a modular norm if and only if it is equivalent to (defines the same topology as) a uniform norm of $E \otimes F$.

Proof. Suppose that $\alpha$ is equivalent to a uniform norm. Then, without loss of generality we can assume that $\alpha$ is itself uniform. Then (7.1) and Theorem 4.7 above immediately imply that $\alpha$ is modular.

Conversely, suppose that $\alpha$ is a modular norm of $E \otimes F$. We construct a uniform norm $\tilde{\alpha}$ of $E \otimes F$ which is equivalent to $\alpha$. For $\mathrm{t} \in E \otimes F$ we define $\tilde{\alpha}(\mathrm{t})$ as

$$
\tilde{\alpha}(\mathrm{t})=\sup \alpha[V \otimes W(\mathrm{t})],
$$

where $V$ ranges over the unit sphere of the operator algebra $\mathscr{L}(E)$ and $W$ over the unit sphere of $\mathscr{L}(F)$. It follows from Theorem 4.6 that $\tilde{\alpha}(\mathrm{t})<+\infty$. Clearly $\tilde{\alpha}$ is a norm of $E \otimes F$ which is greater than, or equal to, $\alpha$. It again follows from Theorem 4.6 that $\tilde{\alpha}$ is bounded with respect, hence equivalent, to $\alpha$. It remains to show that $\tilde{\alpha}$ is uniform. Let $V_{1} \in \mathscr{L}(E), W_{1} \in \mathscr{L}(F)$ and $\mathrm{t} \in E \otimes F$. Then

$$
\begin{aligned}
\tilde{\alpha}\left[V_{1} \otimes W_{1}(\mathrm{t})\right] & =\sup \alpha\left\{V \otimes W\left[V_{1} \otimes W_{1}(\mathrm{t})\right]\right\}=\sup \alpha\left[V V_{1} \otimes W W_{1}(\mathrm{t})\right] \\
& \leqq\left\|V_{1}\right\|\left\|W_{1}\right\| \sup \alpha[\bar{V} \otimes \bar{W}(\mathrm{t})]=\left\|V_{1}\right\|\left\|W_{1}\right\| \tilde{\alpha}(\mathrm{t}) .
\end{aligned}
$$

In (7.4) all suprema taken as $V$ and $\bar{V}$ vary over the unit sphere of $\mathscr{L}(E)$, and $W$ and $\bar{W}$ vary over the unit sphere of $\mathscr{L}(F)$. This completes our proof.

It is of interest to note that the process employed in the proof of the above theorem preserves the property of being a cross norm.

COROLlaRY 7.1. Let $\alpha$ be a modular cross norm of $E \otimes F$. Then $\alpha$ is equivalent to a uniform cross norm $\tilde{\alpha}$ of $E \otimes F$.

Proof. It suffices to show that if $\alpha$ is a cross norm, the passage to $\tilde{\alpha}$ in Theorem 7.1 preserves this property. Let $x \in E$ and $y \in F$. We have already seen from the proof of the Theorem 7.1, that $\tilde{\alpha} \geqq \alpha$. Hence we have, in particular

$$
\tilde{\alpha}(x \otimes y) \geqq \alpha(x \otimes y)=\|x\|\|y\| .
$$

We show the reverse inequality.

$$
\tilde{\alpha}(x \otimes y)=\sup \alpha(V x \otimes W y)=\sup \|V x\|\|W y\| \leqq\|x\|\|y\|,
$$

where all the suprema are taken as $V$ and $W$ range over the respective unit spheres of $\mathscr{L}(E)$ and $\mathscr{L}(F)$. This completes our proof.

A similar argument will yield the following corollary.

COROLlaRY 7.2. Let $\alpha$ be a modular ordinary norm of $E \otimes F$. Then $\alpha$ is equivalent to a uniform ordinary norm of $E \otimes F$. 
In our work in [7] we have shown that every uniform ordinary norm on the tensor product $A \otimes B$ of two Banach algebras $A$ and $B$ gives rise to an ordinary norm $\bar{\alpha}$ of $A \otimes B$, which is compatible (see $\S 1$ ) with the multiplication defined on $A \otimes B$ by extending the product

$$
\left(U_{1} \otimes V_{1}\right)\left(U_{2} \otimes V_{2}\right)=U_{1} U_{2} \otimes V_{1} V_{2}
$$

for $U_{1}, U_{2} \in A$ and $V_{1}, V_{2} \in B$, in such a way that one can generalize to the Banach algebra $A \otimes_{\bar{\alpha}} B$ the theory developed by Gelbaum [4], [5] and Tomiyama [15] for $A \otimes_{\gamma} B$. In the same work we have also given a generalization to uniform cross norms of a result of the author and B. R. Gelbaum on Schauder bases of tensor products, obtained originally in [6] only for $\lambda$ and $\gamma$. We shall devote the remaining portion of the present section to show that in view of Theorem 7.1, this entire development can be extended, without any essential loss, to norms $\alpha$ which are merely modular, and that the assumption that $\alpha$ is ordinary can be dispensed with entirely. We begin by reviewing briefly the basic constructions developed in [7]. We refer the reader to that paper for details. We shall assume that both $A$ and $B$ have identity elements. This is a simplifying, but not essential, assumption, (as we have shown in [7]), which we feel justified to make here because our purpose is to illustrate the passage from the earlier setting to the present one, rather than to achieve the utmost generality.

The algebra $A$ can be imbedded isometrically in $\mathscr{L}(A)$ and similarly for $B$ and $\mathscr{L}(B)$, by means of the left regular representation. In what follows the elements of $A$ and $B$ will be made to play dual roles, one as vectors in the algebras, and the other as bounded linear operators on the algebras, by virtue of the left regular representation. Let now $\alpha$ be a modular norm of $A \otimes B$. We have seen in $\S 4$, that a $U \in A$ and a $V \in B$, as bounded linear operators, define a Kronecker product $U \otimes V$, a bounded operator on $A \otimes_{\alpha} B$. In this manner, by identifying the tensor product $A \otimes B$, with the Kronecker product $A \otimes B$, the validity of such an identification having been established in [10], we can imbed the tensor product $A \otimes B$ into the algebra of operators $\mathscr{L}\left(A \otimes_{\alpha} B\right)$. It was shown in [10] that this is an imbedding of algebras, and that in case that $\alpha$ is a uniform cross norm, the operator norm of the algebra $\mathscr{L}\left(A \otimes_{\alpha} B\right)$ induces a norm $\bar{\alpha}$ on $A \otimes B$ as a subalgebra of $\mathscr{L}\left(A \otimes_{\alpha} B\right)$, which is a cross norm, obviously compatible with multiplication on $A \otimes B$. Hence $A \otimes_{\bar{\alpha}} B$ is a Banach algebra. If $\alpha$ is only a modular norm, the operator norm on $\mathscr{L}\left(A \otimes_{\alpha} B\right)$ still induces a norm $\bar{\alpha}$ on $A \otimes B$, which is compatible with multiplication, although $\bar{\alpha}$ will not, of course, be, in general, a cross norm.

THEOREM 7.2. Let $\alpha$ be a modular norm of $A \otimes B$. Then $\bar{\alpha}$ is admissible. If $\alpha$ is, in addition, ordinary, then $\bar{\alpha}$ is also ordinary.

Proof. From the definition of admissibility in $\S 3$, one has to show that $\bar{\alpha}$ majorizes the least cross norm $\lambda$ on $A \otimes B$ and is majorized by the greatest cross norm $\gamma$ 
on $A \otimes B$. We have from Theorem 4.5 that $\alpha$ itself is admissible. We show that $\gamma$ majorizes the $\bar{\alpha}$. Now if we use as a model the proof of Theorem 4.5 , where we established that $\alpha$ is admissible, we see that it suffices to show that there exists a constant $k_{1}>0$ such that, for every $U \in A$ and $V \in B$ we have

$$
\bar{\alpha}(U \otimes V) \leqq k_{1}\|U\|\|V\| .
$$

Now the left of (7.8) is the operator norm of the operator $U \otimes V$. Let $\mathfrak{T} \in A \otimes_{\alpha} B$. Then it follows from relation (4.10), following Theorem 4.6, that

$$
\alpha[U \otimes V(\mathfrak{T})] \leqq k_{1}\|U\|\|V\| \alpha(\mathfrak{T})
$$

from which (7.8) follows immediately.

We now show that $\bar{\alpha}$ majorizes the least cross norm $\lambda$ on $A \otimes B$. We need from Theorem 4.5 the fact that $\alpha$ itself majorizes $\lambda$ on $A \otimes B$. Hence, there is a constant $k_{1}>0$, such that for every $\mathfrak{T} \in A \otimes B$,

$$
\lambda(\mathfrak{T}) \leqq k_{1} \alpha(\mathfrak{T}) .
$$

Let now $I$ be the identity of $A$ and $J$ that of $B$. In the equation $\mathfrak{T}(I \otimes J)=\mathfrak{T}$, we shall interpret $\mathfrak{I}$ on the left as an operator of $\mathscr{L}\left(A \otimes_{\alpha} B\right)$ acting on the tensor $I \otimes J$ of $A \otimes_{\alpha} B$, and on the right as the tensor $\mathfrak{I}$ of $A \otimes B$. Thus we have

$$
\lambda(\mathfrak{I})=\lambda[\mathfrak{I}(I \otimes J)] \leqq k_{1} \alpha[\mathfrak{I}(I \otimes J)] \leqq k_{1} \bar{\alpha}(\mathfrak{I}) \alpha(I \otimes J)
$$

which is what we wanted to show.

The proof that, if $\alpha$ is ordinary, then $\bar{\alpha}$ is also ordinary, follows so closely the proof of the corresponding Theorem 2.1 of [10] that we need not include it here. This concludes our discussion of the present theorem.

We can now summarize the major points of the theory of the Banach algebras $A \otimes_{\bar{\alpha}} B$, for modular norms $\alpha$, in the following theorem. We note that, if $U^{\prime}$ and $V^{\prime}$ are multiplicative continuous linear functionals on $A$ and $B$, respectively, they are, in particular elements of $A^{\prime}$ and $B^{\prime}$, respectively. Hence $U^{\prime} \otimes V^{\prime}$ is an element of $A^{\prime} \otimes B^{\prime}$, which can be imbedded in $\left(A \otimes_{\bar{\alpha}} B\right)^{\prime}$, since $\bar{\alpha}$ is admissible. For the purpose of the next theorem, and only for that purpose, we abandon our convention concerning kernels and write $A^{\prime} \otimes B^{\prime} \subset\left(A \otimes_{\bar{\alpha}} B\right)^{\prime}$ and $U^{\prime} \otimes V^{\prime} \in\left(A \otimes_{\bar{\alpha}} B\right)^{\prime}$.

THEOREM 7.3. Let $A$ and $B$ be any two Banach algebras with identity elements and $\alpha$ a modular norm of $A \otimes B$. Then $\bar{\alpha}$ is an admissible norm of the algebra $A \otimes B$, which is compatible with multiplication. The mapping $\left(U^{\prime}, V^{\prime}\right) \rightarrow U^{\prime} \otimes V^{\prime}$ effects a homeomorphism of the cartesian product $M_{A} \times M_{B}$, of the spaces of multiplicative linear functionals on $A$ and $B$ into the corresponding space for $A \otimes_{\bar{\alpha}} B$, when all such spaces are taken with their respective weak* topologies. If $A$ and $B$ are commutative and semisimple then $A \otimes_{\bar{\alpha}} B$ is commutative and semisimple if $\alpha$ is faithful (see §3). 
Proof. We simply indicate those aspects that are different from the arguments in [10]. We have already seen that $\bar{\alpha}$ is an admissible norm that is compatible with multiplication. The imbedding of $M_{A} \times M_{B}$ into the multiplicative-functional space of $A \otimes_{\bar{\alpha}} B$ is always a homeomorphism which is onto, provided that the tensors of the form $U^{\prime} \otimes V^{\prime}$ do indeed belong to $\left(A \otimes_{\bar{\alpha}} B\right)^{\prime}$. This is where the assumption that $\alpha$ is ordinary was used in [10]. However, we dispense with this assumption here, because we know now, and we did not know when [10] was written, that $\bar{\alpha}$ is admissible. Hence the functionals defined by the tensors $U^{\prime} \otimes V^{\prime}$ are continuous with respect to $\bar{\alpha}$. The last assertion of the theorem follows exactly as in Theorem 2.3 of [10]. This completes our discussion of the present theorem.

If $\alpha$ is a modular cross norm we have no guarantee that $\bar{\alpha}$ is a cross norm, unless $\alpha$ is also uniform [10, Theorem 2.1]. However $\bar{\alpha}$ is always equivalent to an admissible cross norm compatible with multiplication, if $\alpha$ is a modular cross norm. To see this we merely make use of Corollary 7.1 above, replacing $\alpha$ by an equivalent uniform cross norm, and then apply the theory developed in [10]. We emphasize once more that the discussion of algebras $A$ and $B$ with identity extends easily to the general case when no identity is assumed to exist.

Another problem discussed in [10] was the existence of a Schauder basis for the tensor product of spaces $E$ and $F$ with Schauder bases. A biorthogonal system constructed elsewhere [6] out of the biorthogonal systems defining bases for $E$ and $F$ was proved in [10] to define a basis for $E \otimes_{\alpha} F$ for $\alpha$ uniform and ordinary. This result extends now to modular norms. Finally Corollary 4.1 of [10] states that if $\alpha$ is uniform and ordinary, then it is faithful, provided that $E$ and $F$ have Schauder bases. In the light of our discussion of this section, we can again remove the assumption that $\alpha$ must be a cross norm hence, that it is ordinary, to obtain the following simple result.

COROLlaRY 7.3. Let $E$ and $F$ be Banach spaces with Schauder bases and $\alpha a$ modular norm of $E \otimes F$. Then $\alpha$ is faithful.

8. Algebras of kernels. In $\S 3$ we have seen that a tensor $t \in E \otimes F$ can be identified with a bounded linear transformation $T_{\mathrm{t}}: E^{\prime} \rightarrow F$, of which we have said it is a kernel. We have called the mapping $\mathrm{t} \rightarrow T_{\mathrm{t}}$ the natural imbedding of $E \otimes F$ into $\mathscr{L}\left(E^{\prime}, F\right)$. We have seen in the same section that if $\alpha$ is an admissible norm of $E \otimes F$, then the natural imbedding can be extended to a bounded linear transformation $\Psi^{\alpha}: E \otimes_{\alpha} F \rightarrow \mathscr{L}\left(E^{\prime}, F\right)$, which may no longer be isomorphic. We still refer to $\mathrm{t} \in E \otimes_{\alpha} F$ as a kernel of $T_{\mathrm{t}}=\Psi^{\alpha} \mathrm{t}$. Let now $\alpha$ be a modular norm of $E \otimes F$. We have seen (Theorem 4.5) that $\alpha$ is indeed admissible. Therefore any tensor of $E \otimes_{\alpha} F$, where $\alpha$ is modular, is a kernel of a bounded linear transformation of $E^{\prime}$ into $F$.

In this section we are going to specialize our considerations to the tensor product $E^{\prime} \otimes E$, where $E^{\prime}$ is, as usual, the dual (conjugate) space of $E$. We are going to assume, without further mention, that $\alpha$ is a modular norm of $E^{\prime} \otimes E$. By the above 
discussion, any tensor $\mathfrak{v} \in E^{\prime} \otimes_{\alpha} E$ is a kernel of a bounded linear transformation of $E^{\prime \prime}$ into $E$. Since this transformation is continuous with respect to the weak* topology of $E^{\prime \prime}$ and the weak topology of $E(\S 3)$, it is completely determined by the values it assumes on $E \subset E^{\prime \prime}$. Consequently, for convenience, we are going to identify this transformation with its restriction $V_{\mathfrak{v}}$ to $E$ and change our conventions in order to refer to $\mathfrak{v}$ as a kernel of the operator $V_{\mathfrak{b}} \in \mathscr{L}(E)$. We are also going to refer systematically to the tensors of $E^{\prime} \otimes_{\alpha} E$ as kernels. We are going to define a product of kernels on $E^{\prime} \otimes_{\alpha} E$, which will turn the latter space into a Banach algebra. Any such algebra will be called an algebra of kernels. The product of two kernels will be defined so as to correspond to composition of operators, i.e., so that the mapping $\mathfrak{v} \rightarrow V_{\mathfrak{v}}$ of $E^{\prime} \otimes_{\alpha} E$ into $\mathscr{L}(E)$ is a homomorphism of algebras. Consequently, in the cases in which $\alpha$ is faithful, i.e., the mapping $\mathfrak{v} \rightarrow V_{\mathfrak{b}}$ is isomorphic, the study of algebras of kernels reduces to the study of algebras of operators.

Let us now define the product of two kernels. For $x^{\prime}, u^{\prime} \in E^{\prime}$ and $x, u \in E$ we define

$$
\left(x^{\prime} \otimes x\right)\left(u^{\prime} \otimes u\right)=\left\langle u, x^{\prime}\right\rangle u^{\prime} \otimes x .
$$

From (8.1) we get the definition of the product of any two finite tensors, at least formally, according to the distributivity law. Since the generators $x^{\prime} \otimes x$ of $E^{\prime} \otimes E$ are not, in general, linearly independent, it remains to be shown that our definition makes sense, namely that the product $\mathfrak{v}_{1} \mathfrak{v}_{2}$ of two kernels $\mathfrak{v}_{1}$ and $\mathfrak{v}_{2}$ of $E^{\prime} \otimes E$ is a kernel which is independent of the particular representations of the individual kernels in terms of the generators $x^{\prime} \otimes x$. But the proof of this follows the general pattern of proofs of similar facts about algebraic tensor products, and we omit the details. To complete our definition, we have to extend this product to the entire $E^{\prime} \otimes_{\alpha} E$, but before we do this we need the following lemmas.

LEMMA 8.1. If $\alpha$ is an ordinary uniform norm, then $\alpha$ is compatible with the product defined by (8.1).

Proof. Let $\mathfrak{v}_{1}$ and $\mathfrak{v}_{2}$ be kernels in $E^{\prime} \otimes E$. We must show that $\alpha\left(\mathfrak{v}_{1} \mathfrak{v}_{2}\right) \leqq \alpha\left(\mathfrak{y}_{1}\right) \alpha\left(\mathfrak{y}_{2}\right)$. Now $\mathfrak{v}_{1}$ is a kernel of an operator $V_{\mathfrak{b}_{1}}$. Simple algebraic manipulations yield the following relations, for the interpretation of which one must refer back to $\S 4$, in particular the relations (4.1) and (4.12).

$$
\mathfrak{v}_{1} \mathfrak{b}_{2}=\mathfrak{v}_{2} \cdot V_{\mathfrak{v}_{1}}=I \otimes V_{\mathfrak{v}_{1}}\left(\mathfrak{v}_{2}\right),
$$

where $I$ here represents the identity operator of $E$. From (8.2), the uniformity of $\alpha$ and the fact that $\alpha$ is ordinary we get

$$
\begin{aligned}
\alpha\left(\mathfrak{v}_{1} \mathfrak{y}_{2}\right)=\alpha\left(V_{\mathfrak{y}_{1}} \cdot \mathfrak{v}_{2}\right) & \leqq\left\|V_{\mathfrak{v}_{1}}\right\| \alpha\left(\mathfrak{v}_{2}\right)=\lambda\left(\mathfrak{v}_{1}\right) \alpha\left(\mathfrak{y}_{2}\right) \\
& \leqq \alpha\left(\mathfrak{v}_{1}\right) \alpha\left(\mathfrak{y}_{2}\right) .
\end{aligned}
$$

This completes our proof. 
The assumption that $\alpha$ is uniform and ordinary permitted the result of Lemma 8.1 to be formulated in neat and familiar terms. But it is plain that this assumption is not essential to the topological properties involved. In fact, for a norm $\alpha$ which is merely modular, we have the following lemma.

LEMMA 8.2. Let $\alpha$ be a modular norm of $E^{\prime} \otimes E$. Then the product defined by (8.1) on $E^{\prime} \otimes E$ is continuous with respect to $\alpha$.

Proof. We know from Theorem 4.5 that $\alpha$ is admissible. Hence, there exists a number $\kappa>0$, such that $\alpha(\mathfrak{b}) \geqq \kappa \lambda(\mathfrak{b})$ for every $\mathfrak{v} \in E^{\prime} \otimes E$. Now for any two $\mathfrak{v}_{1}$ and $\mathfrak{v}_{2}$ in $E^{\prime} \otimes E$, as in the proof of Lemma 8.1, we have that (8.2) and (8.3) are still valid, except that in (8.3) one must write $\kappa \alpha\left(\mathfrak{b}_{1}\right) \alpha\left(\mathfrak{y}_{2}\right)$ instead of the last term on the extreme right. This completes the desired proof of continuity.

It follows from Lemma 8.2 that the product defined by (8.1) on $E^{\prime} \otimes E$ can now be extended to a continuous multiplication on $E^{\prime} \otimes_{\alpha} E$. We now have the following theorem.

THEOREM 8.1. Every modular norm $\alpha$ of $E^{\prime} \otimes E$ is equivalent to a modular norm $\beta$ of $E^{\prime} \otimes E$ which is compatible with multiplication. Consequently $E^{\prime} \otimes_{\alpha} E$ is a Banach algebra under $\beta$.

Proof. The pattern here is the familiar one. We know that $E^{\prime} \otimes_{\alpha} E$ is a Banach space with a continuous multiplication, under which it is an algebra. One first imbeds $E^{\prime} \otimes_{\alpha} E$ isometrically into a Banach space which is an algebra $\mathscr{A}$ with identity, whose multiplication is continuous with respect to the norm. One can then imbed $E^{\prime} \otimes_{\alpha} E$ isomorphically into $\mathscr{L}(\mathscr{A})$ by means of the left regular representation. It is well known that the norm $\beta$ induced by the operator norm in $\mathscr{L}(\mathscr{A})$ on $E^{\prime} \otimes_{\alpha} E$ is both compatible with multiplication and equivalent to $\alpha$. The property of modularity is a topological property and is passed on to $\beta$ by $\alpha$, This completes our proof.

It follows then from Theorem 8.1 that a modular norm on the algebra $E^{\prime} \otimes E$ can be replaced by an equivalent modular norm which is compatible with our multiplication. Therefore, we can assume, without loss of generality that $\alpha$ is itself such a norm, i.e., that $\alpha$ is compatible with multiplication and that $E^{\prime} \otimes_{\alpha} E$ is a Banach algebra under the product defined by (8.1). We shall make this assumption in the rest of the present section, without further notice. It should be remarked that if $\alpha$ is a cross norm, we have no guarantee that the passage from $\alpha$ to $\beta$ preserves this property $\left({ }^{10}\right)$. We now proceed with the study of the Banach algebra $E^{\prime} \otimes_{\alpha} E$.

Since we are assuming that $\alpha$ is modular we see that the tensor product $E^{\prime} \otimes_{\alpha} E$ comes within the scope of the theory developed in $\$ \$ 4$ through 7. Hence $\left(E^{\prime} \otimes_{\alpha} E\right)^{\prime}$ is a subspace of $\mathscr{L}\left(E^{\prime}\right)$, and for $T \in\left(E^{\prime} \otimes_{\alpha} E\right)^{\prime}$ and $\mathfrak{v} \in E^{\prime} \otimes_{\alpha} E$ the products

$\left({ }^{10}\right)$ This, and other similar situations, have led us to abandon cross norms in the present theory, in favor of a topological substitute, that of tensorial norm, defined in $\$ 3$. 
$T \times \mathfrak{v} \in E^{\prime} \otimes_{\pi} E^{\prime \prime}$ and $\mathfrak{v} \times T \in E^{\prime} \bigotimes_{\pi} E$ are well defined. In the same way, if $T \in \mathscr{L}\left(E^{\prime}\right)$ and $V \in \mathscr{L}(E)$, the Kronecker product $T \otimes V$ is defined as an operator of $\mathscr{L}\left(E^{\prime} \otimes_{\alpha} E\right)$, and, therefore so are the products $T \cdot \mathfrak{v}$ and $\mathfrak{v} \cdot V$, as elements of $E^{\prime} \otimes_{\alpha} E$, given $\mathfrak{v} \in E^{\prime} \otimes_{\alpha} E$. One establishes easily that, for $T \in \mathscr{L}\left(E^{\prime}\right), V \in \mathscr{L}(E)$ and $\mathfrak{v}_{1}, \mathfrak{v}_{2} \in E^{\prime} \otimes_{\alpha} E$, we have

$$
T \cdot\left(\mathfrak{v}_{1} \mathfrak{b}_{2}\right) \cdot V=\left(\mathfrak{v}_{1} \cdot V\right)\left(T \cdot \mathfrak{b}_{2}\right) .
$$

The method of proof for any such relation is the one so often employed above, and consists of establishing the desired relation for finite tensors first and then passing to the limit. We note also that the relation

$$
\mathfrak{v}_{1} \mathfrak{v}_{2}=\mathfrak{v}_{2} \cdot V_{\mathfrak{v}_{1}},
$$

which we have already seen for finite kernels $\mathfrak{v}_{1}$ and $\mathfrak{v}_{2}$, extends to any two kernels in $E^{\prime} \otimes_{\alpha} E$. We also have the corresponding equation

$$
\mathfrak{v}_{1} \mathfrak{y}_{2}=V_{\mathfrak{b}_{2}}^{\prime} \cdot \mathfrak{v}_{1}
$$

The next result now follows easily from equations (8.5) and (8.6). We omit the proof. We refer the reader to $\$ 4$ for our conventions and notations regarding $\mathscr{L}(E)$-left and $\mathscr{L}(F)$-right submodules of a tensor product $E \otimes_{\alpha} F$.

Corollary 8.1. Every $\mathscr{L}\left(E^{\prime}\right)$-left and $\mathscr{L}(E)$-right submodule of the tensor product $E^{\prime} \otimes_{\alpha} E$ is a two-sided ideal of the Banach algebra $E^{\prime} \otimes_{\alpha} E$.

We now discuss the relation between the faithful character of $\alpha$ (that is, the question whether $\Psi^{\alpha}$ is isomorphic) and the Jacobson radical of the Banach algebra $E^{\prime} \otimes_{\alpha} E$. It was discovered by Gelbaum [5] that the radical of the tensor product $A \otimes_{\gamma} B$ of two commutative semisimple Banach algebras $A$ and $B$ is precisely the null space of $\Psi^{\alpha}$. It follows from our own work in [10] and the supplementary discussion in $\$ 7$ above that this result extends to the tensor product (see $\S 7$, above) $A \otimes_{\bar{\alpha}} B$, where we no longer have the $\gamma$ norm, but any norm $\bar{\alpha}$ associated with a modular norm $\alpha$. It is of interest, and indeed a surprise, that the same result holds for the Jacobson radical of the Banach algebra $E^{\prime} \otimes_{\alpha} E$, for a norm $\alpha$, which is again modular, but where otherwise the context is so completely different. In the first place, we do not have Banach algebras as initial ingredients, only a Banach space and its dual. In the second place, the product of kernels is so completely foreign to the multiplication (7.7) in the tensor product of Banach algebras, even when $E^{\prime}$ and $E$ are Banach algebras. In the third place, in the present context there is, in general, complete lack of commutativity. The result in question here follows directly from Theorem 2.8.21 of [13] and we incorporate it with other facts in the following theorem. We have defined our product of kernels so that the mapping $\Psi^{\alpha}: E^{\prime} \bigotimes_{\alpha} E \rightarrow \mathscr{L}(E)$ is a homomorphism of algebras. Let us denote by $\mathscr{N}_{\alpha}$ the null space of $\Psi^{\alpha}$. Then, since $\Psi^{\alpha}$ is continuous, $\mathscr{N}_{\alpha}$ is a closed two-sided ideal. By the left (right) annihilator of $E^{\prime} \otimes_{\alpha} E$ we shall mean the set of all those kernels $\mathfrak{v}$ such that $\mathfrak{v}_{1}\left(\mathfrak{v}_{1} \mathfrak{b}\right)$ is 0 , for every $\mathfrak{v}_{1} \in E^{\prime} \bigotimes_{\alpha} E$. 
THEOREM 8.2. The null space $\mathscr{N}_{\alpha}$ of the mapping $\Psi^{\alpha \alpha}: E^{\prime} \bigotimes_{\alpha} E \rightarrow \mathscr{L}(E)$ is the Jacobson radical, the left annihilator and the right annihilator of $E^{\prime} \otimes_{\alpha} E$.

Proof. Since the mapping $\Psi^{\alpha}$ restricted to the algebraic tensor product $E^{\prime} \otimes E$ is an isomorphism of algebras, the complete tensor product $E^{\prime} \otimes_{\alpha} E$ comes within the scope of Theorem 2.8 .21 of [13, p. 103]. From this it follows that $\mathscr{N}_{\alpha}$ is the Jacobson radical of $E^{\prime} \otimes_{\alpha} E$. To complete our proof we show that $\mathscr{N}_{\alpha}$ is the annihilator, on either side, of $E^{\prime} \otimes_{\alpha} E$. In the first place we see that the algebraic tensor product $E^{\prime} \otimes E$ is a two-sided ideal of $E^{\prime} \otimes_{\alpha} E$, because the product of any kernel by a kernel of finite rank is a kernel of finite rank. Let then $\mathfrak{v} \in \mathscr{N}_{\alpha}$ and

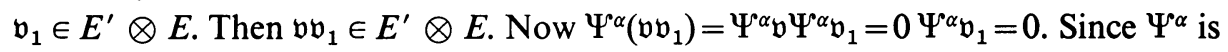
isomorphic on $E^{\prime} \otimes E$, it follows that $\mathfrak{v b}_{1}=0$. In the same manner one shows that $\mathfrak{v}_{1} \mathfrak{v}=0$. We see that $\mathscr{N}_{\alpha}$ annihilates the algebraic tensor product $E^{\prime} \otimes E$ on both sides. Since $E^{\prime} \otimes E$ is dense in $E^{\prime} \otimes_{\alpha} E$, then $\mathscr{N}_{\alpha}$ annihilates the entire $E^{\prime} \otimes_{\alpha} E$ on both sides. Suppose conversely that $\mathfrak{v} \in E^{\prime} \otimes_{\alpha} E$ and that $\mathfrak{v}$ annihilates $E^{\prime} \otimes_{\alpha} E$ on the left (right). Then, in particular, for every $x^{\prime} \in E$ and $x \in E$,

$$
\mathfrak{v}\left(x^{\prime} \otimes x\right)=x^{\prime} \otimes V_{\mathfrak{v}} x=0 \quad\left(\left(x^{\prime} \otimes x\right) \mathfrak{v}=V_{\mathfrak{v}}^{\prime} x^{\prime} \otimes x=0\right),
$$

from which we conclude easily that $V_{\mathfrak{v}}=0$. Hence $\mathfrak{v} \in \mathscr{N}_{\alpha}$. This completes our proof.

COROLlaRY 8.2. The modular norm $\alpha$ on $E^{\prime} \otimes E$ is faithful if and only if the algebra of kernels $E^{\prime} \otimes_{\alpha} E$ is semisimple.

Proof. $\alpha$ is faithful if and only if $\mathscr{N}_{\alpha}$ is trivial, if and only if $E^{\prime} \otimes_{\alpha} E$ is semisimple.

The next theorem appears as a remark on p. 102 of [13], but we have not been able to find a proof in the literature $\left({ }^{11}\right)$. We give here such a proof for the sake of completeness. For a discussion of topological simplicity see $\$ 2$ above.

THEOREM 8.3. Let $\mathscr{A}$ be a subalgebra of $\mathscr{L}(E)$ which contains the ideal $\mathscr{F}$ of all bounded linear operators of finite rank. Suppose that $\mathscr{A}$ is a Banach algebra with respect to some norm \|\|$_{1}$, with respect to which $\mathscr{F}$ is dense in $\mathscr{A}$. Then the Banach algebra $\mathscr{A}$ is topologically simple.

Proof (suggested orally by B. R. Gelbaum). Clearly, it suffices to show that any nontrivial two-sided ideal $\mathscr{I}$ of $\mathscr{A}$ contains $\mathscr{F}$, or, what amounts to the same, contains every operator $V_{x^{\prime} \otimes x}$ on $E$ with kernel $x^{\prime} \otimes x$, for $x^{\prime} \in E^{\prime}$ and $x \in E$. Fix $x^{\prime} \in E^{\prime}, x \in E, x \neq 0, x^{\prime} \neq 0$ and $V \in \mathscr{I}, V \neq 0$. Then there must exist $x_{0}^{\prime} \in E^{\prime}$ such that $V_{x_{0}^{\prime} \otimes x} V \neq 0$. Otherwise, it would follow that $V=0$. Hence, as $u$ varies over $E$, the relation $\left\langle u, u^{\prime}\right\rangle=\left\langle V u, x_{0}^{\prime}\right\rangle$ defines a nonzero linear functional $u^{\prime} \in E^{\prime}$. Then $V_{u^{\prime} \otimes x}=V_{x_{0}^{\prime} \otimes x} V \in \mathscr{I}$. Let now $N$ be the hyperplane on which the functional $u^{\prime}$ vanishes and $u_{0} \in E$ such that $\left\langle u_{0}, u^{\prime}\right\rangle=1$. Then $V_{x^{\prime} \otimes x}=V_{u^{\prime} \otimes x} V_{x^{\prime} \otimes u_{0}} \in \mathscr{I}$. This completes our proof.

(11) It is stated in [13] that the result is obvious. 
CoROllary 8.3. The modular norm $\alpha$ on $E^{\prime} \otimes E$ is faithful if and only if the algebra of kernels $E^{\prime} \otimes_{\alpha} E$ is topologically simple.

Proof. If $E^{\prime} \otimes_{\alpha} E$ is topologically simple, then the closed two-sided ideal $\mathscr{N}_{\alpha}$ is trivial and $\Psi^{\alpha}$ is isomorphic. Hence $\alpha$ is faithful. Conversely, if $\alpha$ is faithful, $\Psi^{\alpha}$ maps $E^{\prime} \bigotimes_{\alpha} E$ isomorphically onto a subalgebra $\mathscr{A}$ of $\mathscr{L}(E)$ and the algebraic tensor product $E^{\prime} \otimes E$ onto $\mathscr{F}$. This isomorphism induces a norm on $\mathscr{A}$, under which it is a Banach algebra, isometric with $E^{\prime} \otimes_{\alpha} E$. The hypotheses of Theorem 8.2 are satisfied. It follows that $\mathscr{A}$, hence $E^{\prime} \otimes_{\alpha} E$, is topologically simple.

THEOREM 8.4. The radical $\mathscr{N}_{\alpha}$ contains every proper two-sided closed ideal of the algebra of kernels $E^{\prime} \otimes_{\alpha} E$ hence is the only proper maximal closed two-sided ideal.

Proof. The proof is very much like that of Theorem 8.3 , and we merely sketch it. Clearly, it suffices to show that if $\mathscr{I}$ is a two-sided ideal which is not contained in $\mathscr{N}_{\alpha}$, then $\mathscr{I}$ is dense in $E^{\prime} \otimes_{\alpha} E$, and for this it suffices to show that the algebraic tensor product $E^{\prime} \otimes E$ is contained in $\mathscr{I}$. So let $\mathscr{I}$ be a two-sided ideal of $E^{\prime} \otimes_{\alpha} E$ not contained in $\mathscr{N}_{\alpha}$. We simply have to show that every $x^{\prime} \otimes x$, for $x^{\prime} \in E^{\prime}$ and $x \in E$, belongs to $\mathscr{I}$. First, (Theorem 8.2) there exists a $\mathfrak{v} \in \mathscr{I}$, which does not annihilate $E^{\prime} \otimes_{\alpha} E$, say, on the right. This actually means that $\mathfrak{v}$ does not annihilate the algebraic tensor product $E^{\prime} \otimes E$ on the right. This means, as in the proof of Theorem 8.3 that there exists an $x_{0}^{\prime} \in E^{\prime}$ so that $V_{x_{0}^{\prime} \otimes x} V \neq 0$. Again, if $u^{\prime} \in E^{\prime}$ is defined, for $u \in E$ by the equation $\left\langle u, u^{\prime}\right\rangle=\left\langle V_{\mathfrak{v}} u, x_{0}^{\prime}\right\rangle, u^{\prime} \neq 0$. If we now choose $u \in E$ so that $\left\langle u, u^{\prime}\right\rangle=1$, then we have, in a similar way as in Theorem 8.3, (keeping in mind equation (8.5))

$$
\begin{aligned}
\left(x_{0}^{\prime} \otimes x\right) \mathfrak{v}\left(x^{\prime} \otimes u\right) & =\left(x_{0}^{\prime} \otimes x\right)\left(x^{\prime} \otimes V_{\mathfrak{b}} u\right) \\
& =\left\langle V_{\mathfrak{b}} u, x_{0}^{\prime}\right\rangle x^{\prime} \otimes x=\left\langle u, u^{\prime}\right\rangle x^{\prime} \otimes x \\
& =x^{\prime} \otimes x .
\end{aligned}
$$

It follows from (8.7) that $x^{\prime} \otimes x \in \mathscr{I}$, as we wanted to prove.

COROLlary 8.4. The algebra of kernels $E^{\prime} \otimes_{\alpha} E$ is semisimple if and only if it is topologically simple.

Proof. This is a direct consequence of Corollaries 8.2 and 8.3.

In the light of the above discussion, the structure of the two-sided ideals of the algebra of kernels $E^{\prime} \otimes_{\alpha} E$ appears clear. We summarize this structure in the following theorem.

THEOREM 8.5. If the space $E$ is nontrivial (i.e., if its dimension exceeds 0 ) the set of all proper closed two-sided ideals of the algebra of kernels $E^{\prime} \otimes_{\alpha} E$ is precisely the lattice of all closed vector subspaces of the radical $\mathscr{N}_{\alpha}$. The radical $\mathscr{N}_{\alpha}$ is the only proper closed maximal two-sided ideal.

Proof. The last assertion of the theorem has already appeared in Theorem 8.4. That theorem also asserts that any proper closed two-sided ideal of $E^{\prime} \otimes_{\alpha} E$ is a 
vector subspace of $\mathscr{N}_{\alpha}$. Conversely, since $\mathscr{N}_{\alpha}$ is the left and the right annihilator of $E^{\prime} \otimes_{\alpha} E$, any subspace of it is a two-sided ideal. This completes our proof.

In the case in which $E$ is a Hilbert space $H$, in which case $E^{\prime}$ is also $H$, it is known that the norms $\lambda, \gamma$ and the Schmidt norm $\sigma$ are faithful and uniform (see [14, Chapter 5]). It follows then, from Theorem 8.4 that the trace class $H \otimes_{\gamma} H$, the algebra of completely continuous operators $H \otimes_{\lambda} H$, and the Schmidt class of operators $H \otimes_{\sigma} H$ are topologically simple algebras, since in each case $\mathscr{N}_{\alpha}$ is trivial. This fact was discovered by Rickart [13, Appendix]. We can now establish a more general result.

COROLlary 8.5. Suppose that $E^{\prime}$ has a Schauder basis and that $\alpha$ is a modular norm of $E^{\prime} \otimes E$. Then the Banach algebra $E^{\prime} \otimes_{\alpha} E$ is topologically simple.

Proof. It is well known and follows easily from [2, p. 69, Theorem 2] that if $E^{\prime}$ has a Schauder basis, so does $E$. It now follows from Corollary 7.3, that, if $\alpha$ is modular, then it is faithful. From this our conclusion follows, since, then $\mathscr{N}_{\alpha}$ is trivial.

The next theorem gives a simple description of all strictly irreducible representations of $E^{\prime} \otimes_{\alpha} E$, for $\alpha$ modular, which is a consequence of the above discussion of the Jacobson radical $\mathscr{N}_{\alpha}$. We refer the reader to [13, Chapter II] for details of some of the concepts involved. However we begin by reviewing some of the basic definitions. An algebra of operators on a (not necessarily topological) vector space is said to be strictly irreducible if the only vector spaces left setwise invariant by it are $\{0\}$ and the entire space. A (not necessarily continuous) representation of an algebra as an algebra of operators is said to be strictly irreducible if the representing algebra of operators has this property. We shall be concerned with the representation $\Psi^{\alpha}: E^{\prime} \otimes_{\alpha} E \rightarrow \mathscr{L}(E)$. Let us denote by $\mathscr{A}$ the algebra which is the image of $E^{\prime} \otimes_{\alpha} E$ under $\Psi^{\alpha}$. Clearly $\mathscr{A}$ is isomorphic to the quotient algebra of $E^{\prime} \otimes_{\alpha} E$ modulo $\mathscr{N}_{\alpha}$ and the quotient norm of the latter induces a norm $\tilde{\alpha}$ on $\mathscr{A}$, which renders the natural isomorphism isometric. We have, by Theorem 8.3, that $\mathscr{A}$ is a topologically simple Banach algebra under $\tilde{\alpha}$.

THEOREM 8.6. The representation $\Psi^{\alpha}: E^{\prime} \otimes_{\alpha} E \rightarrow \mathscr{L}(E)$ is strictly irreducible. Furthermore, any continuous strictly irreducible representation $\Omega$ of $E^{\prime} \otimes_{\alpha} E$ into the algebra $\mathscr{L}(F)$ of all bounded operators on any Banach space, is either trivial (i.e., $F$ is trivial) or equivalent to $\Psi^{\alpha}$, in the sense that there exists an isomorphism $\Xi: \mathscr{A} \rightarrow \mathscr{L}(F)$, in such a way that the following diagram commutes,

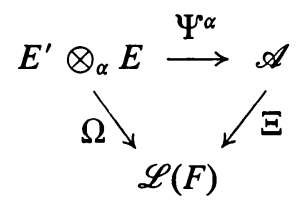

and so that $\Xi$ is continuous with respect to $\tilde{\alpha}$ and the operator norm of $\mathscr{L}(F)$. 
Proof. The fact that $\mathscr{A}$ is strictly irreducible is discussed in detail in [13, Chapter II] and depends on the fact that it contains the space $\mathscr{F}$ of all bounded linear operators on $E$ of finite rank, and that any two points of $E$, different from 0 are interchanged by such operators. Let now $\Omega: E^{\prime} \otimes_{\alpha} E \rightarrow \mathscr{L}(F)$ be any continuous strictly irreducible representation of $E^{\prime} \otimes_{\alpha} E$ for some Banach space $F$. Let $\mathscr{M}$ be the null space of $\Omega$. By one of the several equivalent definitions of the Jacobson radical it follows that $\mathscr{M} \supset \mathscr{N}_{\alpha}$. From this we conclude that there exists a homomorphism $\Xi: \mathscr{A} \rightarrow \mathscr{L}(F)$ of algebras, rendering the above diagram commutative. We claim that $\Xi$ is of necessity continuous with respect to the norm $\tilde{\alpha}$ of $\mathscr{A}$ and the operator norm of $\mathscr{L}(F)$, for this is equivalent to boundedness, and, if $P$ is a bounded set of $\mathscr{A}$, then there exists a bounded set subset $Q$ of $E^{\prime} \otimes_{\alpha} E$, such that $\Psi^{\alpha}(Q)=P$. Hence $\Xi(P)=\Omega(Q)$, a bounded subset of $\mathscr{L}(F)$, since $\Omega$ is bounded. This implies that $\Xi$ is bounded, as claimed. This implies, in turn, that the null space $\mathscr{M}_{1}$ of $\Xi$ is a two-sided ideal of $\mathscr{A}$, which is closed with respect to $\tilde{\alpha}$. Now we have already seen that $\mathscr{A}$ is topologically simple with respect to $\tilde{\alpha}$. Hence $\mathscr{M}_{1}$ is either $\mathscr{A}$, in which case $\Omega$ is the 0 representation, and $F$ is trivial, because of the strict irreducibility of $\Omega$, or $\mathscr{M}_{1}=\{0\}$, in which case $\Xi$ is a continuous isomorphism. This completes the proof of the theorem.

We have already seen that $\mathscr{N}_{\alpha}$ is a closed two-sided ideal of $E^{\prime} \otimes_{\alpha} E$. We are soon going to give a considerably stronger result, which we shall need in the next section. We now lay the groundwork for it. Let $V$ be any operator of $\mathscr{L}(E)$. Its transpose operator belongs to $\mathscr{L}\left(E^{\prime}\right)$ and its double transpose $V^{\prime \prime}$ belongs to $\mathscr{L}\left(E^{\prime \prime}\right)$. It is well known that the mapping $V \rightarrow V^{\prime \prime}$ effects an isometric imbedding of $\mathscr{L}(E)$ into $\mathscr{L}\left(E^{\prime \prime}\right)$. This imbedding composed with $\Psi^{\alpha}$ yields the mapping $\mathfrak{v} \rightarrow V_{\mathfrak{b}}^{\prime \prime}$. We see from this discussion that $\mathfrak{v} \in \mathscr{N}_{\alpha}$ if and only if $V_{\mathfrak{v}}^{\prime \prime}=0$. We now have the following formulas, which will be needed in the next theorem, and which are easily established in the usual manner: first for finite kernels, then passing to the limit.

$$
\begin{aligned}
\left(\mathfrak{v}_{1} \cdot V\right) \mathfrak{v}_{2} & =\mathfrak{v}_{1}\left(V^{\prime} \cdot \mathfrak{v}_{2}\right), \\
V_{T \cdot \mathfrak{b}}^{\prime \prime} & =V_{\mathfrak{v}}^{\prime \prime} T^{\prime} .
\end{aligned}
$$

In (8.8) and (8.9) we take $\mathfrak{v}, \mathfrak{v}_{1}$, and $\mathfrak{v}_{2} \in E^{\prime} \otimes_{\alpha} E, V \in \mathscr{L}(E)$ and $T \in \mathscr{L}\left(E^{\prime}\right)$. We are now ready to state our next theorem. For conventions regarding submodules of a module, see $\$ 4$.

THEOREM 8.7. The Jacobson radical $\mathscr{N}_{\alpha}$ of $E^{\prime} \otimes_{\alpha} E$ is an $\mathscr{L}\left(E^{\prime}\right)$-left and an $\mathscr{L}(E)$-right submodule of $E^{\prime} \otimes_{\alpha} E$.

Proof. To prove that $\mathscr{N}_{\alpha}$ is an $\mathscr{L}\left(E^{\prime}\right)$-left submodule of $E^{\prime} \otimes_{\alpha} E$, we use the characterization of $\mathscr{N}_{\alpha}$ as the null space of $\Psi^{\alpha}$ and the discussion immediately preceding this theorem to conclude that $\mathfrak{v} \in \mathscr{N}_{\alpha}$ if and only if $V_{\mathfrak{v}}^{\prime \prime}=0$. Let $\mathfrak{v} \in \mathscr{N}_{\alpha}$ and $T \in \mathscr{L}\left(E^{\prime}\right) . V_{T \cdot \mathfrak{v}}^{\prime \prime}=V_{\mathfrak{v}}^{\prime \prime} T^{\prime}=0$. From this, we have that $T \cdot \mathfrak{v} \in \mathscr{N}_{\alpha}$. This shows that $\mathscr{N}_{\alpha}$ is an $\mathscr{L}\left(E^{\prime}\right)$-left submodule of $E^{\prime} \otimes_{\alpha} E$. To show that it is also an 
$\mathscr{L}(E)$-right submodule, we use Theorem 8.2. Let $V \in \mathscr{L}(E)$, and $\mathfrak{v} \in \mathscr{N}_{\alpha}$. Let $\mathfrak{v}_{1}$ be any other kernel in $E^{\prime} \otimes_{\alpha} E$. Then, by $(8.8),(\mathfrak{v} \cdot V) \mathfrak{v}_{1}=\mathfrak{v}\left(V^{\prime} \cdot \mathfrak{v}_{1}\right)=0$. Hence, $\mathfrak{v} \cdot V$ annihilates $E^{\prime} \otimes_{\alpha} E$ on the left, and must belong to $\mathscr{N}_{\alpha}$. This completes our proof.

9. Duality of algebras of kernels. In the last section we considered the algebra of kernels $E^{\prime} \otimes_{\alpha} E$, where $\alpha$ is a modular norm, and gave a complete description of its closed two-sided ideals. In this section we turn our attention to the dual space $\left(E^{\prime} \otimes_{\alpha} E\right)^{\prime}$ of $E^{\prime} \otimes_{\alpha} E$. This space is of interest because it also turns out to be a Banach algebra under a norm which is equivalent to the dual norm $\alpha^{\prime}$. Thus in the study of algebras of kernels we are faced with the phenomenon, up to equivalent norms, of algebras whose dual spaces are themselves Banach algebras. This phenomenon occurs, of course, in many known cases, such as group algebras, and has already been noted with regard to certain tensor products by Schatten [14]. Here we give a completely general theory. Our aim, as before, is to give as complete a description as we can of the ideal structure of the dual kernel algebras.

Our first aim is to establish the dual space $\left(E^{\prime} \otimes_{\alpha} E\right)^{\prime}$ as a Banach algebra. We know $(\S 3)$ that $\left(E^{\prime} \otimes_{\alpha} E\right)^{\prime}$ is a vector subspace of the Banach algebra $\mathscr{L}\left(E^{\prime}\right)$. Now the definition of modularity of $\alpha$ requires that $\left(E^{\prime} \otimes_{\alpha} E\right)^{\prime}$ be invariant under left multiplication by elements of $\mathscr{L}^{\#}\left(E^{\prime}\right)$ and under right multiplication by elements of $\mathscr{L}\left(E^{\prime}\right)$, where multiplication is, as usual, composition of operators. It follows immediately that $\left(E^{\prime} \otimes_{\alpha} E\right)^{\prime}$ is a subalgebra of $\mathscr{L}\left(E^{\prime}\right)$, because, if $T_{1}, T_{2} \in\left(E^{\prime} \otimes_{\alpha} E\right)^{\prime}$, then $T_{2} \in \mathscr{L}\left(E^{\prime}\right)$, and $T_{1} T_{2} \in\left(E^{\prime} \otimes_{\alpha} E\right)^{\prime}$ by the modular character of $\left(E^{\prime} \otimes_{\alpha} E\right)^{\prime}$. Our first aim is to show that multiplication by composition, a well defined operation of $\left(E^{\prime} \otimes_{\alpha} E\right)^{\prime}$ is continuous with respect to $\alpha^{\prime}$.

THEOREM 9.1. The composition of operators is a continuous multiplication on $\left(E^{\prime} \otimes_{\alpha} E\right)^{\prime}$. Hence $\alpha^{\prime}$ is equivalent to a norm, which is compatible with composition of operators, under which $\left(E^{\prime} \otimes_{\alpha} E\right)^{\prime}$ is a Banach algebra. This algebra contains the algebra $\mathscr{H}$ of all bounded linear operators on $E^{\prime}$ of finite rank.

Proof. We have already seen that $\left(E^{\prime} \otimes_{\alpha} E\right)^{\prime}$ is closed under operator composition, hence it is an algebra. We have also seen that it contains the algebra of operators of finite rank since this algebra is nothing but the canonical image of $E^{\prime \prime} \otimes E^{\prime}$ in $\mathscr{L}\left(E^{\prime}\right)$. It simply remains to show that the product of operators is continuous with respect to $\alpha^{\prime}$. We do this. Let $T_{1}, T_{2} \in\left(E^{\prime} \otimes_{\alpha} E\right)^{\prime}$. We now use Theorem 4.2 on the product $T_{1} T_{2}$ by treating $T_{2}$ as an element of the algebra $\mathscr{L}\left(E^{\prime}\right)$ and $T_{1}$ as an element of the module $\left(E^{\prime} \otimes_{\alpha} E\right)^{\prime}$. We then obtain, from relation (4.2), that $\alpha^{\prime}\left(T_{1} T_{2}\right) \leqq k_{1}\left\|T_{2}\right\| \alpha^{\prime}\left(T_{1}\right)$, where $k_{1}$ depends only on $\alpha$, and not on the operators $T_{1}$ and $T_{2}$. Now, we know that $\alpha^{\prime}$ majorizes the operator norm. Hence we obtain, for some constant $\kappa>0$,

$$
\alpha^{\prime}\left(T_{1} T_{2}\right) \leqq \kappa k_{1} \alpha^{\prime}\left(T_{1}\right) \alpha^{\prime}\left(T_{2}\right)
$$

from which the desired continuity follows. 
Since $\alpha^{\prime}$ is equivalent to a norm on $\left(E^{\prime} \otimes_{\alpha} E\right)^{\prime}$ which is compatible with multiplication, $\left(E^{\prime} \otimes_{\alpha} E\right)^{\prime}$ is a Banach algebra with respect to that norm, which, for our purposes here, will remain in the background. That norm may be different from $\alpha^{\prime}$, except in certain cases, some of which are indicated in the following corollary.

COROLlary 9.1. If $\alpha$ is a uniform cross norm of $E^{\prime} \otimes E$, then $\alpha^{\prime}$ is itself compatible with the multiplication in $\left(E^{\prime} \otimes_{\alpha} E\right)^{\prime}$.

Proof. We establish this simply by noticing that in the computations involved in the proof of Theorem 9.1, $k \leqq 1$, if $\alpha$ is uniform, and that $\kappa \leqq 1$ if $\alpha \leqq \gamma$. But we have $\alpha \leqq \gamma$, because $\alpha$ is a cross norm. This completes our argument.

COROLlary 9.2. If $\alpha$ is a uniform ordinary cross norm, then both $\alpha$ and $\alpha^{\prime}$ are compatible with multiplication. In this case we can assert that both $E^{\prime} \otimes_{\alpha} E$ and $\left(E^{\prime} \otimes_{\alpha} E\right)^{\prime}$ are Banach algebras under their natural norms.

Proof. This is nothing but a summary of Lemma 8.1 and Corollary 9.1.

We now return to the general case and study the Banach algebra $\left(E^{\prime} \otimes_{\alpha} E\right)^{\prime}$ for a modular norm $\alpha$.

COROLLARY 9.3. The Banach algebra $\left(E^{\prime} \otimes_{\alpha} E\right)^{\prime}$ is a strictly irreducible algebra of operators.

Proof. This follows from Theorem 9.1, according to which $\left(E^{\prime} \otimes_{\alpha} E\right)^{\prime}$ contains the natural image of $E^{\prime \prime} \otimes E^{\prime}$, which is a strictly irreducible algebra of operators.

We have seen (Theorem 8.7) that the Jacobson radical $\mathscr{N}_{\alpha}$ of $E^{\prime} \otimes_{\alpha} E$ is an $\mathscr{L}\left(E^{\prime}\right)$-left and an $\mathscr{L}(E)$-right submodule of $E^{\prime} \otimes_{\alpha} E$, that is to say, it is left invariant by Kronecker products $T \otimes V$, for $T \in \mathscr{L}\left(E^{\prime}\right)$ and $V \in \mathscr{L}(E)$. It follows from Corollary 6.4 that the polar set $\mathscr{N}_{\alpha}^{0}$ is an $\mathscr{L}^{\#}\left(E^{\prime}\right)$-left and an $\mathscr{L}(E)$-right weakly* closed submodule of $\left(E^{\prime} \otimes_{\alpha} E\right)^{\prime}$. In particular it remains invariant under multiplication on the right by elements of $\mathscr{L}\left(E^{\prime}\right)$, hence it is a right ideal of $\left(E^{\prime} \otimes_{\alpha} E\right)^{\prime}$. We are actually going to show that it is a two-sided ideal in certain special cases. At the moment we are going to give a more concrete description of it which is valid in general. We now lay the groundwork for it.

We have already seen that $\left(E^{\prime} \otimes_{\alpha} E\right)^{\prime}$ contains the natural image of $E^{\prime \prime} \otimes E^{\prime}$ in $\mathscr{L}\left(E^{\prime}\right)$. Let us call this image $\mathscr{H}$. It is the ideal of all bounded linear operators on $E^{\prime}$ of finite rank. By virtue of the imbedding $E \subset E^{\prime \prime}$, we can consider the tensor product $E \otimes E^{\prime}$ as a subspace of $E^{\prime \prime} \otimes E^{\prime}$. We shall denote its natural image in $\mathscr{L}\left(E^{\prime}\right)$ by $\mathscr{G}$. We then have

$$
\mathscr{G} \subset \mathscr{H} \subset\left(E^{\prime} \otimes_{\alpha} E\right)^{\prime} \subset \mathscr{L}\left(E^{\prime}\right) .
$$

We know that, by virtue of these imbeddings, the norm $\alpha^{\prime}$ induces a norm on $E^{\prime \prime} \otimes E^{\prime}$ (hence on $E \otimes E^{\prime}$ ), which we have continued to denote by $\alpha^{\prime}$. We have already seen that $\alpha^{\prime}$ is admissible on $E^{\prime \prime} \otimes E^{\prime}$, (Theorem 4.5), hence on $E \otimes E^{\prime}$. If $\alpha$ happens to be an ordinary norm, then so is $\alpha^{\prime}$, this last result being due to 
Schatten [14]. Let us now denote by $\mathscr{H}_{\alpha}$ and $\mathscr{G}_{\alpha}$ the respective closures of $\mathscr{H}$ and $\mathscr{G}$ in $\left(E^{\prime} \otimes_{\alpha} E\right)^{\prime}$. Now $\mathscr{H}_{\alpha}$ is a closed two-sided ideal of $\left(E^{\prime} \otimes_{\alpha} E\right)^{\prime}$, as can be easily verified. It should also be noted that, while $\mathscr{G}_{\alpha}$ is not, in general, a two-sided ideal of $\left(E^{\prime} \otimes_{\alpha} E\right)^{\prime}$, it is a closed subalgebra of $\left(E^{\prime} \otimes_{\alpha} E\right)^{\prime}$.

In the discussion that we will soon present it will be convenient to have available the following elementary lemma, which we do not seem to be able to find in the standard references. In the canonical imbedding of $E$ into $E^{\prime \prime}$, we know that the solid sphere $S_{r} \subset E$, of radius $r>0$ about 0 , is weakly* dense in the corresponding sphere $S_{r}^{\prime \prime}$ of $E^{\prime \prime}$. The following lemma is a slight sharpening of this result. Its present form was suggested to the author by B. R. Gelbaum and J. T. Joichi, to replace an earlier result of the author which was weaker and had a more complicated proof.

LEMMA 9.1. Let $r>0$ and $R_{r}=\{x \mid x \in E,\|x\|=r\}$. Then, in the context of the canonical imbedding of $E$ into $E^{\prime \prime}, R_{r}$ is weakly* dense in the corresponding set $R_{r}^{\prime \prime}=\left\{x^{\prime \prime} \mid x^{\prime \prime} \in E^{\prime \prime},\left\|x^{\prime \prime}\right\|=r\right\}$. If $E$ is infinite dimensional $R_{r}$ is actually weakly* dense in $S_{r}^{\prime \prime}$.

Proof. Clearly we can assume that $E$ is infinite dimensional. Let $x_{0}^{\prime \prime} \in S_{r}^{\prime \prime}$ and $M$ a weak* neighborhood of $x_{0}^{\prime \prime}$. We must show that there exists a point $x \in R_{r}$ which is contained in $M$. Now since $S_{r}$ is weakly* dense in $S_{r}^{\prime \prime}$, we can find $x_{0} \in S_{r}$ which is contained in $M$. Now there exists a weak* neighborhood $N$ of $0 \in E^{\prime \prime}$, such that $x_{0}+N \subset M$. Now $N \cap E$ contains an infinite dimensional subspace of $E$, in particular a vector $h \neq 0$. Then, for some value of the scalar parameter $\theta$, $\left\|x_{0}+\theta h\right\|=r$, and $x_{0}+\theta h \in M \cap R_{r}$. This completes our proof.

LEMMA 9.2. The weak* closure of $\mathscr{G}_{\alpha}$ in $\left(E^{\prime} \otimes_{\alpha} E\right)^{\prime}$ coincides with the weak* closure of $\mathscr{H}_{\alpha}$.

Proof. Since we have $\mathscr{G} \subset \mathscr{H}$, we also have that the weak* closure of $\mathscr{G}_{\alpha}$ is contained in that of $\mathscr{H}_{\alpha}$. The converse is what remains to be shown. After repeated applications of the Hahn-Banach theorem, one concludes that this boils down to showing that, for every $\mathfrak{v} \in E^{\prime} \otimes_{\alpha} E$ and every $T \in \mathscr{H}$, the number $\langle\mathfrak{v}, T\rangle$ can be approximated arbitrarily closely by the number $\left\langle\mathfrak{v}, T_{1}\right\rangle$, with $T_{1}$ this time belonging to $\mathscr{G}$. So let us fix a $\mathfrak{v} \in E^{\prime} \otimes_{\alpha} E, T \neq 0$ in $\mathscr{H}$, and $\varepsilon>0$. Now $T$ has a kernel $\mathfrak{y} \in E^{\prime \prime} \otimes E$, and we may pick a representation

so that (see (3.4))

$$
\mathfrak{y}=\sum_{i=1}^{n} x_{i}^{\prime \prime} \otimes x_{i}^{\prime}
$$

$$
\gamma(\mathfrak{y})>\sum_{i=1}^{n}\left\|x_{i}^{\prime \prime}\right\|\left\|x_{i}^{\prime}\right\|-\varepsilon
$$

We also have that the norm $\alpha^{\prime}$ on $E^{\prime \prime} \otimes E$ is majorized by $\gamma$ on $E^{\prime \prime} \otimes E$. This means that there exists a constant $k>0$, such that

$$
\alpha^{\prime}(\overline{\mathfrak{y}}) \leqq k \gamma(\overline{\mathfrak{y}}) \text {, }
$$


for every $\overline{\mathfrak{y}} \in E^{\prime \prime} \otimes E$. Let us now consider our kernel $\mathfrak{v} \in E^{\prime} \otimes_{\alpha} E$. We can write

$$
\mathfrak{v}=\mathfrak{v}_{1}+\mathfrak{v}_{2}
$$

where

$$
\mathfrak{v}_{1}=\sum_{j=1}^{m} u_{j}^{\prime} \otimes u_{j}
$$

is a finite kernel of $E^{\prime} \otimes E$, and $\alpha\left(\mathfrak{y}_{2}\right)<\varepsilon$. We now use Lemma 9.1 to find vectors $x_{1}, x_{2}, \cdots, x_{n}$, in $E$ such that $\left\|x_{i}\right\|=\left\|x_{i}^{\prime \prime}\right\|$ and that

$$
\left|\sum_{i, j}\left\langle u_{j}^{\prime}, x_{i}^{\prime \prime}\right\rangle\left\langle u_{j}, x_{i}^{\prime}\right\rangle-\sum_{i, j}\left\langle x_{i}, u_{j}^{\prime}\right\rangle\left\langle u_{j}, x_{i}^{\prime}\right\rangle\right|<\varepsilon .
$$

Finally we define $\mathfrak{y}_{1} \in E \otimes E^{\prime}$ by the relation

$$
\mathfrak{y}_{1}=\sum_{i=1}^{n} x_{i} \otimes x_{i}
$$

as the kernel of $T_{1} \in \mathscr{G}$. We now have all the ingredients for our approximation. Let us now compute

$$
\left|\left\langle\mathfrak{v}, T-T_{1}\right\rangle\right| \leqq\left|\left\langle\mathfrak{v}_{1}, T-T_{1}\right\rangle\right|+\left|\left\langle\mathfrak{v}_{2}, T\right\rangle\right|+\left|\left\langle\mathfrak{v}_{2}, T_{1}\right\rangle\right| .
$$

Now $\left\langle\mathfrak{v}_{1}, T-T_{1}\right\rangle$ is precisely the quantity on the left side of (9.8). Hence we have the following estimate

$$
\left|\left\langle\mathfrak{v}, T-T_{1}\right\rangle\right| \leqq \varepsilon+\alpha\left(\mathfrak{y}_{2}\right) \alpha^{\prime}(T)+\alpha\left(\mathfrak{b}_{2}\right) \alpha^{\prime}\left(T_{1}\right) .
$$

We now make reference to the estimates (9.5) and (9.6), to get the further estimate

$$
\left|\left\langle\mathfrak{v}, T-T_{1}\right\rangle\right| \leqq \varepsilon+\varepsilon \alpha^{\prime}(T)+k \varepsilon \sum_{i=1}^{n}\left\|x_{i}\right\|\left\|x_{i}^{\prime}\right\| .
$$

Finally, by the choice $\left\{x_{i}\right\}$, we get the estimate

$$
\left|\left\langle\mathfrak{y}, T-T_{1}\right\rangle\right| \leqq \varepsilon+\varepsilon \alpha^{\prime}(T)+k \varepsilon[\gamma(\mathfrak{y})+\varepsilon],
$$

representing a fixed small number. This completes the proof.

THEOREM 9.2. The polar set $\mathscr{N}_{\alpha}^{\circ}$ of the Jacobson radical $\mathscr{N}_{\alpha}$ of $E^{\prime} \otimes_{\alpha} E$ is the weak* closure of $\mathscr{H}_{\alpha}$ and the weak* closure of $\mathscr{G}_{\alpha}$.

Proof. We have already seen that the weak* closure of $\mathscr{G}_{\alpha}$ is that of $\mathscr{H}_{\alpha}$. It remains to show that it is also $\mathscr{N}_{\alpha}^{\circ}$. We have, by Theorem 8.7 , that $\mathscr{N}_{\alpha}$ is an $\mathscr{L}\left(E^{\prime}\right)$-left and an $\mathscr{L}(E)$-right submodule of $E^{\prime} \otimes_{\alpha} E$. It follows from Corollary 6.3 that $\mathscr{N}_{\alpha}^{\circ}$ is an $\mathscr{L}^{\#}\left(E^{\prime}\right)$-left and an $\mathscr{L}\left(E^{\prime}\right)$-right submodule of $\left(E^{\prime} \otimes_{\alpha} E\right)^{\prime}$, hence also of $\mathscr{L}\left(E^{\prime}\right)$. We now apply Theorem 4.1 to get that $\mathscr{N}_{\alpha}^{\circ}$ must contain $\mathscr{H}$. Since $\mathscr{N}_{\alpha}^{\circ}$ is weakly* closed, it must also contain the weak* closure of $\mathscr{H}_{\alpha}$.

We now show the reverse inclusion. To do this we make use of the double polar theorem already quoted in $\$ 6$. We refer the reader to our discussion in that section for details. Let us denote by $\mathscr{G}^{-}$the polar set in $E^{\prime} \otimes_{\alpha} E$ of the set $\mathscr{G}$. We show that $\mathscr{G}-\subset \mathscr{N}_{\alpha}$. Let $\mathfrak{v} \in \mathscr{G}^{-}$. Then, in particular, for every $x \in E$ and $x^{\prime} \in E^{\prime}$ (see 3.18)

$$
\left\langle\mathfrak{v}, T_{x \otimes x^{\prime}}\right\rangle=\left\langle V_{\mathfrak{b}} x, x^{\prime}\right\rangle=0 .
$$


This implies that $V_{\mathfrak{v}}=0$, and that $\mathfrak{v}$ belongs to $\mathscr{N}_{\alpha}$ the null space of the mapping $\mathfrak{v} \rightarrow V_{\mathfrak{b}}$. This shows that $\mathscr{G}^{-} \subset \mathscr{N}_{\alpha}$. We conclude that $\mathscr{G}^{-\circ} \supset \mathscr{N}_{\alpha}^{\circ}$. By the double polar theorem we have that $\mathscr{G}^{-\circ}$ is the weak* closure of $\mathscr{G}_{\alpha}$, hence (Lemma 9.3), that of $\mathscr{H}_{\alpha}$. This completes our proof.

This might be a good place to examine critically what we have established about the Banach algebras $\mathscr{L}\left(E^{\prime}\right),\left(E^{\prime} \otimes_{\alpha} E\right)^{\prime}, \mathscr{G}_{\alpha}$, and $\mathscr{H}_{\alpha} .\left(E^{\prime} \otimes_{\alpha} E\right)^{\prime}$ is a closed subalgebra of $\mathscr{L}\left(E^{\prime}\right)$, which may not be a two-sided ideal of $\mathscr{L}\left(E^{\prime}\right)$, but is an $\mathscr{L}^{\#}\left(E^{\prime}\right)$-left and an $\mathscr{L}\left(E^{\prime}\right)$-right submodule of $\mathscr{L}\left(E^{\prime}\right)$. This means that it is closed under right multiplication by any element of $\mathscr{L}\left(E^{\prime}\right)$ (hence is a right ideal) and on the left by multiplication by any element of $\mathscr{L}^{\#}\left(E^{\prime}\right)$, i.e., by any operator on $E^{\prime}$ which is the transpose of an operator of $E$. If $E$ is reflexive, then $\mathscr{L}\left(E^{\prime}\right)$ and $\mathscr{L}^{\#}\left(E^{\prime}\right)$ coincide and then $\left(E^{\prime} \otimes_{\alpha} E\right)^{\prime}$ is a two-sided ideal of $\mathscr{L}\left(E^{\prime}\right)$. In the same manner $\mathscr{N}_{\alpha}^{\circ}$ is a closed (indeed weakly* closed) $\mathscr{L}^{\#}\left(E^{\prime}\right)$-left and $\mathscr{L}\left(E^{\prime}\right)$-right submodule, in particular, a right ideal of $\left(E^{\prime} \otimes_{\alpha} E\right)^{\prime}$ which may not be a two-sided ideal, for similar reasons. It is a two-sided ideal if $E$ is reflexive. Later on in this section we shall see another less trivial case in which $\mathscr{N}_{\alpha}^{\circ}$ is a two-sided ideal.

Let us now examine the subalgebra $\mathscr{H}_{\alpha}$ of $\mathscr{N}_{\alpha}^{\circ}$. It clearly satisfies the hypothesis of Theorem 8.3 , hence is topologically simple and, therefore, semisimple. Since $E^{\prime \prime} \otimes_{\alpha^{\prime}} E^{\prime}$ is isometric to $\mathscr{H}_{\alpha}$ the same can be said about $E^{\prime \prime} \otimes_{\alpha^{\prime}} E^{\prime}$. The algebra $\mathscr{H}_{\alpha}$ is a two-sided ideal of $\mathscr{N}_{\alpha}^{\circ}$ (indeed, of $\mathscr{L}\left(E^{\prime}\right)$ ), as well as an $\mathscr{L} \#\left(E^{\prime}\right)$-left and an $\mathscr{L}\left(E^{\prime}\right)$-right submodule of $\mathscr{L}\left(E^{\prime}\right)$. Hence, it is a right ideal of $\mathscr{L}\left(E^{\prime}\right)$. However, we do not know in general, whether it is a left ideal of $\mathscr{L}\left(E^{\prime}\right)$. The difference, as usual, is between multiplication on the left by elements of $\mathscr{L}^{\#}\left(E^{\prime}\right)$ and by those of $\mathscr{L}\left(E^{\prime}\right)$. This difference disappears if we assume in addition that $\alpha$ is strongly modular. We shall make this assumption later on in this section, in order to obtain a clearer picture of the ideal structure of $\left(E^{\prime} \otimes_{\alpha} E\right)^{\prime}$, but for the moment, we simply assume, as before, that $\alpha$ is modular.

The corresponding results for $E \otimes_{\alpha^{\prime}} E^{\prime}$ do not seem to be as immediate as those for $E^{\prime \prime} \otimes_{\alpha^{\prime}} E^{\prime}$, but the reasoning is analogous. The main difference is that its corresponding isometric space $\mathscr{G}_{\alpha}$ of operators does not satisfy the hypothesis of Theorem 8.3. However, it does satisfy the hypothesis of a completely analogous result, which we now state without proof.

LEMMA 9.3. Let $\mathscr{B}$ be a subalgebra of $\mathscr{L}\left(E^{\prime}\right)$, which contains $\mathscr{G}$ and is a Banach algebra under some norm (presumably different from the operator norm), with respect to which $\mathscr{G}$ is dense in $\mathscr{B}$. Then $\mathscr{B}$ is topologically simple.

It follows from this lemma that $\mathscr{G}_{\alpha}$, hence $E \otimes_{\alpha^{\prime}} E^{\prime}$, is topologically simple and, therefore, semisimple. $\mathscr{G}_{\alpha}$ is an $\mathscr{L}^{\#}\left(E^{\prime}\right)$-left and an $\mathscr{L}(E)$-right submodule of $\mathscr{N}_{\alpha}^{\circ}$, but is not, in general, a left ideal of $\mathscr{N}_{\alpha}^{\circ}$.

We have already seen that if $\alpha$ is a modular norm of $E^{\prime} \otimes E$, then $\alpha$ is admissible on $E^{\prime} \otimes E$ and $\alpha^{\prime}$ is admissible on $E^{\prime \prime} \otimes E^{\prime}$. We do not know if $\alpha^{\prime}$ is modular on 
$E^{\prime \prime} \otimes E^{\prime}$, but we have the following result. Its proof, which we omit, is similar to that of Theorem 4.8 .

THEOREM 9.3. Suppose that $\alpha$ is a strongly modular norm of $E^{\prime} \otimes E$. Then $\alpha^{\prime}$ is modular on $E \otimes E^{\prime}$.

We now resume our main line of development by establishing the following result.

THEOREM 9.4. Every nontrivial weakly* closed $\mathscr{L}^{\#}\left(E^{\prime}\right)$-left and $\mathscr{L}(E)$-right submodule of $\left(E^{\prime} \otimes_{\alpha} E\right)^{\prime}$ contains $\mathscr{N}_{\alpha}^{\circ}$.

Proof. Let $\mathscr{M}$ be a nontrivial weakly closed $\mathscr{L}^{\#}\left(E^{\prime}\right)$-left and $\mathscr{L}\left(E^{\prime}\right)$-right submodule of $\left(E^{\prime} \otimes_{\alpha} E\right)^{\prime}$, and $\mathscr{M}^{-}$its polar set in $E^{\prime} \otimes_{\alpha} E$. By Corollary $6.4 \mathscr{M}^{-}$ is an $\mathscr{L}\left(E^{\prime}\right)$-left and an $\mathscr{L}(E)$-right submodule of $E^{\prime} \otimes_{\alpha} E$, hence a two-sided ideal (Corollary 8.1). It must be a proper two-sided ideal, otherwise $\mathscr{M}$ is trivial. Thus $\mathscr{M}^{-} \subset \mathscr{N}_{\alpha}$, by Theorem 8.4. We now apply the double polar theorem, [1, p. 52, Proposition 3], which we have quoted earlier in this section to conclude that $\mathscr{M}=\left(\mathscr{M}^{-}\right)^{\circ} \supset \mathscr{N}_{\alpha}^{\circ}$. This concludes our proof.

So far we have only been able to obtain definitive information about weakly* closed $\mathscr{L}^{\#}\left(E^{\prime}\right)$-left, $\mathscr{L}\left(E^{\prime}\right)$-right submodules of $\left(E^{\prime} \otimes_{\alpha} E\right)^{\prime}$ and not about the twosided ideals that are weakly* closed. We are going now to investigate those, but under somewhat more restrictive assumptions. The specific assumptions are that $\alpha$ is not only modular, but also strongly modular, and that $E$ satisfies the condition of approximation of Grothendieck, which we have discussed in $\$ 1$ and elsewhere in this paper. The first assumption means that $\left(E^{\prime} \otimes_{\alpha} E\right)^{\prime}$ is an $\mathscr{L}\left(E^{\prime}\right)$-left and an $\mathscr{L}\left(E^{\prime}\right)$-right submodule of $\mathscr{L}\left(E^{\prime}\right)$. This implies by Theorem 6.6 , that the product $\mathfrak{v} \square T \in E^{\prime} \otimes_{\gamma} E$ is well defined for $\mathfrak{v} \in E^{\prime} \otimes_{\alpha} E$ and $T \in\left(E^{\prime} \otimes_{\alpha} E\right)^{\prime}$ and has the desired continuity properties, which we do not elaborate here. It also follows from the discussion following Theorem 6.6 that $\mathscr{N}_{\alpha}^{\circ}$ is an $\mathscr{L}\left(E^{\prime}\right)$-left and an $\mathscr{L}\left(E^{\prime}\right)$-right submodule of $\left(E^{\prime} \otimes_{\alpha} E\right)^{\prime}$, hence a two-sided ideal of $\left(E^{\prime} \otimes_{\alpha} E\right)^{\prime}$. The assumption that $E$ satisfies the condition of approximation of Grothendieck means that $\gamma$, the greatest cross norm, is faithful. In our present context this means that $\mathscr{N}_{\gamma}$ is trivial, hence $E^{\prime} \otimes_{\gamma} E$ is topologically simple. We shall use this fact in the following more concrete form: the kernel $\mathfrak{v} \in E^{\prime} \otimes_{\gamma} E$ is 0 if and only if the transformation $V_{\mathfrak{b}}=0$. In the proof of the next theorem we shall need the following equation, which is easily verified for finite kernels, and extended by passing to the limit.

LEMMA 9.4. Let $\alpha$ be a strongly modular norm of $E^{\prime} \otimes E$. Then, for every $\mathfrak{v} \in E^{\prime} \otimes_{\alpha} E, V \in \mathscr{L}(E)$, and $T \in\left(E^{\prime} \otimes_{\alpha} E\right)^{\prime}$, we have the relation

$$
V^{\prime} \square(\mathfrak{v} \square T)=(\mathfrak{v} \cdot V) \square T .
$$

In this lemma it should be noted, that, since $V \in \mathscr{L}(E), \mathfrak{b} \cdot V$ is well defined. Since $V^{\prime} \in \mathscr{L}\left(E^{\prime}\right)=\left(E^{\prime} \otimes_{\gamma} E\right)^{\prime}, \gamma$ satisfies the hypothesis of Theorem 6.12 and $\mathfrak{v} \square T \in E^{\prime} \otimes_{\gamma} E, V^{\prime} \square(\mathfrak{v} \square T)$ is well defined. 
THEOREM 9.5. Suppose that E satisfies the condition of approximation of Grothendieck and that $\alpha$ is a strongly modular norm of $E^{\prime} \otimes E$. Then the polar set of a left ideal $\mathscr{I}$ of $E^{\prime} \otimes_{\alpha} E$ consists of all $T \in\left(E^{\prime} \otimes_{\alpha} E\right)^{\prime}$, which annihilate $\mathscr{I}$ on the right under the $\square$-product. The polar set in $E^{\prime} \otimes_{\alpha} E$ of a right ideal $\mathscr{J}$ of $\left(E^{\prime} \otimes_{\alpha} E\right)^{\prime}$ consists of all $\mathfrak{v} \in E^{\prime} \otimes_{\alpha} E$ which annihilate $\mathscr{J}$ on the left, under the $\square$-product.

Proof. The proofs of the two parts of the theorem are similar. We limit ourselves to discussing the proof for a left ideal of $E^{\prime} \otimes_{\alpha} E$. Suppose that $T \in\left(E^{\prime} \otimes_{\alpha} E\right)^{\prime}$ belongs to the right annihilator of $\mathscr{I}$ under the $\square$-product. Then $T \in \mathscr{I}^{\circ}$, for, if $\mathfrak{v} \in \mathscr{I},\langle\mathfrak{v}, T\rangle=\operatorname{tr}(\mathfrak{v} \times T)=0$. We must show the converse. Suppose that $T \in \mathscr{I}^{\circ}$. We show that $\mathfrak{v} \square T=0$, for every $\mathfrak{v} \in \mathscr{I}$. Now $\mathfrak{v} \square T \in E^{\prime} \otimes_{\gamma} E$, and, since $E$ satisfies the condition of approximation of Grothendieck, it suffices to show that $\mathfrak{v} \square T$ is the kernel of the zero operator. Let it be the kernel of some operator $\bar{V} \in \mathscr{L}(E)$. We show $\bar{V}=0$. Let $x \in E$, and $x^{\prime} \in E^{\prime}$. We now compute, using Lemma 9.4. In that lemma, we let $V=V_{x^{\prime} \otimes x}$.

$$
\left\langle\bar{V} x, x^{\prime}\right\rangle=\operatorname{tr}\left[V_{x^{\prime} \otimes x}^{\prime} \times(\mathfrak{v} \times T)\right]=\operatorname{tr}\left[\left(\mathfrak{b} \cdot V_{x^{\prime} \otimes x}\right) \square T\right] .
$$

We now use relation (8.5) to get $\mathfrak{v} \cdot V_{x^{\prime} \otimes x}=\left(x^{\prime} \otimes x\right) \mathfrak{v}$. This and (9.14) give

$$
\left\langle\bar{V} x, x^{\prime}\right\rangle=\left\langle\left(x^{\prime} \otimes x\right) \mathfrak{y}, T\right\rangle=0 .
$$

This completes our proof.

From this theorem, we get the following corollary, whose proof we omit. For a similar proof, see that of Corollary 6.3.

COROLlary 9.4. Suppose that $E$ satisfies the condition of approximation of Grothendieck and that $\alpha$ is a strongly modular norm of $E^{\prime} \otimes E$. Then the polar set of every left ideal of $E^{\prime} \otimes_{\alpha} E$ is a weakly* closed right ideal of $\left(E^{\prime} \otimes_{\alpha} E\right)^{\prime}$ and the polar set in $E^{\prime} \otimes_{\alpha} E$ of a right ideal of $\left(E^{\prime} \otimes_{\alpha} E\right)^{\prime}$ is a closed left ideal of $E^{\prime} \otimes_{\alpha} E$.

We have now information concerning the left ideals of $E^{\prime} \otimes_{\alpha} E$, and by duality, the right ideals of $\left(E^{\prime} \otimes_{\alpha} E\right)^{\prime}$. To obtain information about the ideals on the other side, one must make other assumptions.

THEOREM 9.6. Suppose that E satisfies the condition of approximation of Grothendieck and that $\alpha$ is a norm on $E^{\prime} \otimes E$, which can be extended to a modular norm on $E^{m} \otimes E$. Then the polar set of a right ideal of $E^{\prime} \otimes_{\alpha} E$ is its left annihilator in $\left(E^{\prime} \otimes_{\alpha} E\right)^{\prime}$ under the $\square$-product. The polar set in $E^{\prime} \otimes_{\alpha} E$ of a left ideal of $\left(E^{\prime} \otimes_{\alpha} E\right)^{\prime}$ is its right annihilator in $E^{\prime} \otimes_{\alpha} E$ under the $\square$-product.

The $\square$-product in Theorem 9.6 is the product $T \square \mathfrak{v} \in E^{\prime \prime} \otimes_{\gamma} E^{\prime}$, for $T \in\left(E^{\prime} \otimes_{\alpha} E\right)^{\prime}$ and $\mathfrak{v} \in E^{\prime} \otimes_{\alpha} E$, which is well defined, by Theorem 6.12. We omit the proof of the present theorem because it is completely analogous to that of Theorem 9.5. It uses Theorem 6.12 and the discussion following it, in place of Theorem 6.1 and the theory of $\S 6$ based on Theorem 6.1 .

COROLlARY 9.5. Suppose that E satisfies the condition of approximation of Grothendieck and that $\alpha$ is a norm of $E^{\prime} \otimes E$ which can be extended to a modular 
norm on $E^{m} \otimes E$. Then the polar set of a right ideal of $E^{\prime} \otimes_{\alpha} E$ is a weakly* closed left ideal of $\left(E^{\prime} \otimes_{\alpha} E\right)^{\prime}$ and the polar set in $E^{\prime} \otimes_{\alpha} E$ of a left ideal of $\left(E^{\prime} \otimes_{\alpha} E\right)^{\prime}$ is a closed right ideal of $E^{\prime} \otimes_{\alpha} E$.

We can now establish easily the following three theorems from the above discussion. See also Theorem 8.5.

THEOREM 9.7. Suppose that E satisfies the condition of approximation of Grothendieck and that $\alpha$ is a strongly modular norm of $E^{\prime} \otimes E$, which can be extended to a modular norm of $E^{m} \otimes E$. Then, the polar set in $E^{\prime} \otimes_{\alpha} E$ of every two-sided ideal of $\left(E^{\prime} \otimes_{\alpha} E\right)^{\prime}$ is a vector subspace of $\mathscr{N}_{\alpha}$. The polar set of every vector subspace of $\mathscr{N}_{\alpha}$ is a weakly* closed two-sided ideal of $\left(E^{\prime} \otimes_{\alpha} E\right)^{\prime}$.

THEOREM 9.8. Suppose that $E$ and $\alpha$ satisfy the hypotheses of Theorem 9.7. Then the weak* closure of every two-sided ideal of $\left(E^{\prime} \otimes_{\alpha} E\right)^{\prime}$ is a two-sided ideal of $\left(E^{\prime} \otimes_{\alpha} E\right)^{\prime}$.

THEOREM 9.9. Suppose that $E$ and $\alpha$ satisfy the hypotheses of Theorem 9.7. Then every weakly* closed two-sided ideal of $\left(E^{\prime} \otimes_{\alpha} E\right)^{\prime}$ is either trivial or contains $\mathscr{N}_{\alpha}^{\circ}$.

One might wonder about the relation between the topological simplicity of $E^{\prime} \otimes_{\alpha} E$ and that of its dual algebra. We have that the topological simplicity of $E^{\prime} \otimes_{\alpha} E$ is equivalent to what we might call the weak ${ }^{*}$ simplicity of $\left(E^{\prime} \otimes_{\alpha} E\right)^{\prime}$, as spelled out in the following theorem.

THEOREM 9.10. Suppose that $E$ and $\alpha$ satisfy the hypotheses of Theorem 9.7. Then $E^{\prime} \otimes_{\alpha} E$ is topologically simple if and only if every weakly* closed two-sided ideal of $\left(E^{\prime} \otimes_{\alpha} E\right)^{\prime}$ is either trivial or coincides with $\left(E^{\prime} \otimes_{\alpha} E\right)^{\prime}$.

Proof. Suppose that $E^{\prime} \otimes_{\alpha} E$ is topologically simple. Then $\mathscr{N}_{\alpha}$ is trivial and $\mathscr{N}_{\alpha}^{\circ}=\left(E^{\prime} \otimes_{\alpha} E\right)^{\prime}$. Then every weakly* closed nontrivial two-sided ideal of $\left(E^{\prime} \otimes_{\alpha} E\right)^{\prime}$, must coincide with $\left(E^{\prime} \otimes_{\alpha} E\right)^{\prime}$ by Theorem 9.10 . Suppose conversely that $\left(E^{\prime} \otimes_{\alpha} E\right)^{\prime}$ has the desired property. Then $\mathscr{N}_{\alpha}$ is trivial. The topological simplicity of $E^{\prime} \otimes_{\alpha} E$ follows from Theorem 8.4.

The topological simplicity of $\left(E^{\prime} \otimes_{\alpha} E\right)^{\prime}$ implies much more than the topological simplicity of $E^{\prime} \otimes_{\alpha} E$.

THEOREM 9.11. Suppose that $E$ and $\alpha$ satisfy the hypotheses of Theorem 9.7. Then $\left(E^{\prime} \otimes_{\alpha} E\right)^{\prime}$ is topologically simple if and only if

$$
\left(E^{\prime} \otimes_{\alpha} E\right)^{\prime}=E^{\prime \prime} \otimes_{\alpha^{\prime}} E^{\prime} .
$$

Proof. The equality in (9.16) means an isometry. In keeping with the conventions in this work, it would be more precise to replace $E^{\prime \prime} \otimes_{\alpha^{\prime}} E^{\prime}$ by the space $\mathscr{H}_{\alpha} \subset\left(E^{\prime} \otimes_{\alpha} E\right)^{\prime}$. We have already seen that $\mathscr{H}_{\alpha}$ is topologically simple, and the same follows for $\left(E^{\prime} \otimes_{\alpha} E\right)^{\prime}$ from (9.16). Conversely, if $\left(E^{\prime} \otimes_{\alpha} E\right)^{\prime}$ is topologically simple, it must coincide with $\mathscr{H}_{\alpha}$, since the latter is a closed nontrivial two-sided ideal. This completes our proof. 
10. Some simple applications. In this section we discuss certain aspects of the theory developed above, as they apply to some of the familiar examples in the theory of topological tensor products. We have seen that the greatest cross norm $\gamma$ is strongly modular and satisfies the hypothesis of Theorem 6.12. Whether the algebra $E^{\prime} \otimes_{\gamma} E$ is topologically simple or not, is, of course, equivalent to whether $E$ satisfies the condition of approximation of Grothendieck, which is not known, in general. We do know, however, that the dual algebra $\left(E^{\prime} \otimes_{\gamma} E\right)^{\prime}=\mathscr{L}\left(E^{\prime}\right)$ is not topologically simple, unless $E$ is finite dimensional; otherwise every operator of $\mathscr{L}\left(E^{\prime}\right)$ would be completely continuous. The duality between the closed two-sided ideals of $E^{\prime} \otimes_{\gamma} E$ and the weakly* closed two-sided ideals of $\mathscr{L}\left(E^{\prime}\right)$, described in general at the end of $\$ 9$, is valid for this example. In particular, if $E$ satisfies the condition of approximation of Grothendieck, then $\mathscr{L}\left(E^{\prime}\right)$ has the property that we called (see the paragraph just preceding Theorem 9.10) weak* simplicity.

Let us now consider the least cross norm $\lambda$. It also is strongly modular and satisfies the hypotheses of Theorem 6.12. The algebra $E^{\prime} \otimes_{\lambda} E$ is topologically simple because, in view of the fact that $\lambda$ is really the operator norm, $\mathscr{N}_{\lambda}$ is trivial. Consequently the algebra $\left(E^{\prime} \otimes_{\lambda} E\right)^{\prime}$ is weakly* simple. This algebra has been described above (see the end of $\S 6$ ), following Grothendieck, as the algebra of all integral operators of $\mathscr{L}\left(E^{\prime}\right)$. The question of when $\left(E^{\prime} \otimes_{\lambda} E\right)^{\prime}$ is itself a tensor product (see Theorem 9.11) has been the object of some interest in the literature. It was proved by Schatten [14, Theorem 5.11, p. 116] that $\left(H \otimes_{\lambda} H\right)^{\prime}=H \otimes_{\gamma} H$, if $H$ is a Hilbert space. This was extended by Grothendieck [11, Theorem 8, p. 122] to the tensor product $E \otimes_{\lambda} F$ of two Banach spaces, provided that one of them is reflexive and satisfies this condition of approximation. The author [9], has extended this result in that, by dropping the condition of approximation, but keeping reflexivity, one has $\left(E \otimes_{\lambda} F\right)^{\prime}=E^{\prime} \otimes_{\lambda^{\prime}} F^{\prime}$, although $\lambda^{\prime}$ may no longer be $\gamma$. Now, if one looks at Theorem 9.11 above one sees that $\left(E^{\prime} \otimes_{\lambda} E\right)^{\prime}=E^{\prime \prime} \otimes_{\lambda^{\prime}} E^{\prime}$ is equivalent to the topological simplicity of $\left(E^{\prime} \otimes_{\lambda} E\right)^{\prime}$. We established that theorem under the assumption that $E$ satisfies the condition of approximation, an assumption which is not needed to prove that $\left(E^{\prime} \otimes_{\lambda} E\right)^{\prime}=E \otimes_{\lambda^{\prime}} E^{\prime}$, if $E$ is reflexive. This points to the fact that the hypothesis in Theorem 9.11 might be superfluous, at least for reflexive spaces $\left({ }^{12}\right)$.

Let us now consider a Hilbert space $H$. We shall identify $H^{\prime}$ with $H$ as usual. Furthermore we have established (Corollary 7.3) that every modular norm of $H \otimes H$ is faithful. This means that we can identify every tensor product $H \otimes_{\alpha} H$, for $\alpha$ modular, as well as the dual $\left(H \otimes_{\alpha} H\right)^{\prime}$ with a subalgebra of $\mathscr{L}(H)$, the algebra of all bounded operators of $H$. Thus we shall simply assume here that all such tensor products as well as their duals are actually contained in $\mathscr{L}(H)$. With this interpretation the $\square$-products as well as the products of kernels become ordinary products of operators. We then have the result that an operator $T \in \mathscr{L}(H)$

(12) But at the moment we know of no way of dispensing with it. 
belongs to $\left(H \otimes_{\alpha} H\right)^{\prime}$ if and only if $T V$ is a trace class operator for every $V \in H \otimes_{\alpha} H$, and similarly for the product $V T$. The theorems concerning the relation between polar sets of ideals and annihilators now translate in the present situation in terms of ordinary annihilators. For example we have that, for every two-sided ideal $\mathscr{I}$ of $H \otimes_{\alpha} H$, the polar set $\mathscr{I}^{\circ}$ of $\mathscr{I}$, i.e., the set of all $T \in\left(H \otimes_{\alpha} H\right)^{\prime} \subset \mathscr{L}(H)$, with $\langle V, T\rangle=0$, for every $V \in \mathscr{I}$, is exactly the set of all $T \in\left(H \otimes_{\alpha} H\right)^{\prime}$ such that $V T=T V=0$, for every $V \in \mathscr{I}$.

Note. After the present paper had been accepted for publication it came to the attention of the author that the part of Theorem 8.2 concerning the right and left annihilators appeared in a paper by A. Grothendieck (La theorie de Fredholm, Bull. Soc. Math. France 84 (1956), 319-384) for the special case in which $\alpha$ is the greatest cross norm $\gamma$.

\section{BIBLIOGRAPHY}

1. N. Bourbaki, Eléments de mathématique. Livre V. Espaces vectoriels topologiques, Actualités Sci. Ind. Nos. 1189, 1229, Hermann, Paris, 1953, 1955.

2. M. M. Day, Normed linear spaces, Ergebnisse der Mathematik und Grenzgebiete, Heft 21, Springer, Berlin, 1958.

3. H. R. Fischer, Uber eine Klasse topologischer Tensorprodukte, Math. Ann. 150 (1963), 242-258.

4. B. R. Gelbaum, Tensor products of Banach algebras, Canad. J. Math. 11 (1959), 297-310.

5. - Tensor products and related questions, Trans. Amer. Math. Soc. 103 (1962), 525-548.

6. B. R. Gelbaum and J. Gil de Lamadrid, Bases of tensor products of Banach spaces, Pacific J. Math. 11 (1961), 1281-1286.

7. J. Gil de Lamadrid, Uniform cross norms and tensor products of Banach algebras, Bull. Amer. Math. Soc. 69 (1963), 797-803.

8. - Some simple applications of the closed graph theorem, Proc. Amer. Math. Soc. 15 (1964), 509-510.

9. - Measures and tensors, Trans. Amer. Math. Soc. 114 (1965), 98-121.

10. - Uniform cross norms and tensor products of Banach algebras, Duke Math. J. 32 (1965), 359-368.

11. A. Grothendieck, Produits tensoriels topologiques et espaces nucléaires, Mem. Amer. Math. Soc. No. 16 (1955).

12. E. Hille and R. S. Phillips, Functional analysis and semi-groups, Amer. Math. Soc. Colloq. Publ. Vol. 31, Amer. Math. Soc., Providence, R. I., 1959.

13. C. E. Rickart, General theory of Banach algebras, Van Nostrand, New York, 1960.

14. R. Schatten, $A$ theory of cross spaces, Annals of Mathematics Studies, No. 26, Princeton Univ. Press, Princeton, N. J., 1950.

15. J. Tomiyama, Tensor products of commutative Banach algebras, Tôhoku Math. J. 12 (1960), 147-154.

16. B. Yood, Noncommutative Banach algebras and almost periodic functions, Illinois J. Math. 7 (1963), 305-321.

UNIVERSITY OF MINNESOTA, MiNNEAPOLIS, MinNESOTA 